\title{
Organelle dysfunction modulates cholesterol biosynthesis pathway
}

\author{
Dissertation \\ for the award of the degree \\ "Doctor of Philosophy"
}

Division of Mathematics and Natural Sciences of the Georg-August-Universität Göttingen

\begin{abstract}
within the doctoral program Molecular Biology of Cells of the Georg-August University School of Science (GAUSS)
\end{abstract}

\author{
submitted by \\ Leonardo Gabriel Pereyra \\ from Buenos Aires, Argentina \\ Göttingen 2019
}


Members of the Thesis Advisory Committee

Supervisor, reviewer

Thesis committee, reviewer

Thesis committee

\section{Members of the Examination Board}

Referee

$2^{\text {nd }}$ Referee

$3^{\text {rd }}$ Referee

\section{Dr. Nuno Raimundo}

Department of Cellular Biochemistry, University Medical Center Göttingen

Prof. Dr. Michael Meinecke

Department of Cellular Biochemistry, University Medical Center Göttingen

PD. Dr. Sven Thoms

Department of Pediatrics,

University Medical Center Göttingen

Institute of Pharmacology and Toxicology,

University Medical Center Göttingen

Prof. Dr. D. Doenecke

Department of Molecular Biology,

University Medical Center Göttingen

Prof. Dr. Tiago Outeiro

Department of Neurodegeneration and Restaurative Research, University Medical Center Göttingen 


\section{Affidavit}

I hereby declare that my dissertation, named "Organelle dysfunction modulates cholesterol biosynthesis pathway," was written on my own and with no other sources or aids than quoted.

Göttingen, August $6^{\text {th }}, 2019$

Leonardo Gabriel Pereyra 
"The true delight is in the finding out, rather than in the knowing". Isaac Asimov

"No te des por vencido, ni aún vencido, no te sientas esclavo, ni aún esclavo; trémulo de pavor, piénsate bravo, y acomete feroz, ya mal herido".

Piu Avanti, Almafuerte 


\section{Acknowledgments}

First of all, I would like to thank Dr. Nuno Raimundo for giving me the possibility of doing a Ph.D. in his lab and for the constant support during these years.

I want to thank Prof. Michael Meinecke and Prof. Sven Thoms, both of them members of my Thesis Committee, for the continuous feedback and advice during my Ph.D. work.

I want to thank former lab mate, Lorena; she was always willing to help me and teach me techniques whenever I need it. Also, my actual lab mates, Renata and Kasia, help me a lot, and I will always be thankful with them. I am also thankful with Sindhu, for her help with my microscopy experiments.

Special thanks to Anita and King, both of them were essential for my development as a scientist and as a person. They were always there for me, and if I needed help, I know that I could count on them.

I want to thank my friends: Michele, Kumar, Claudia, Piyal, Diego, Renato, Fede, Peppe, and Francesco. More than friends, they were like a second family for me.

I want to thank my parents, Norma and Quique, my brother, Facu, and my grandma, Eulalia. They are more than $13000 \mathrm{Km}$ from me, but they are always present.

For financial support, I am thankful to Deutscher Akademischer Austauschdienst (DAAD), without their support, I could not do my Ph.D. in Germany. 


\section{Table of Content}

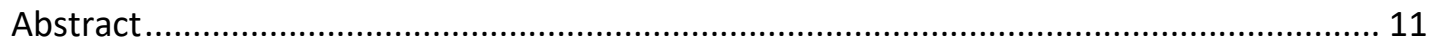

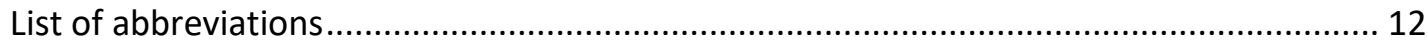

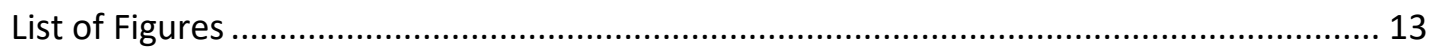

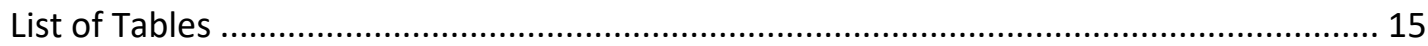

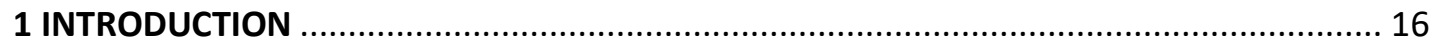

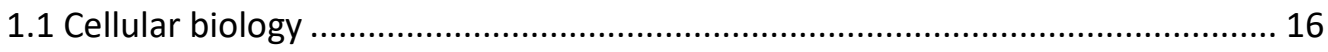

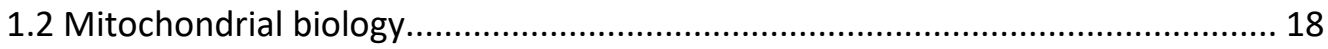

1.2.1 Structure of the mitochondria............................................................ 18

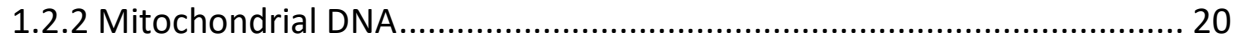

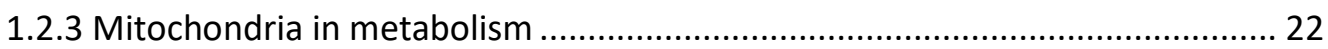

1.2.3.1 Glucose metabolism............................................................. 22

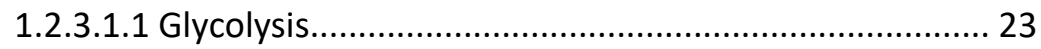

1.2.3.1.2 Citric acid cycle ....................................................... 23

1.2.3.2 Respiratory chain ....................................................... 24

1.2.4 Mitochondrial communication....................................................... 25

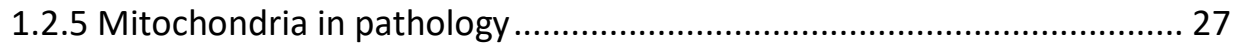

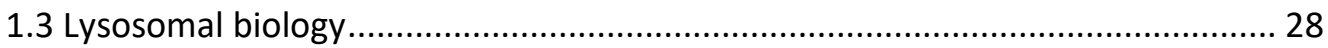

1.3.1 Structure of the lysosome ................................................................ 28

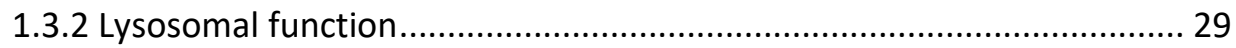

1.3.2.1 Lysosomal degradation of extracellular material ...................... 29

1.3.2.2 Lysosomal degradation of intracellular content ........................ 30

1.3.2.3 Lysosome in nutrient sensing.............................................. 32

1.3.3 Lysosomal storage diseases............................................................ 32

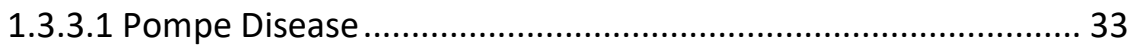

1.3.3.2 Cysteine cathepsins proteases ................................................ 34

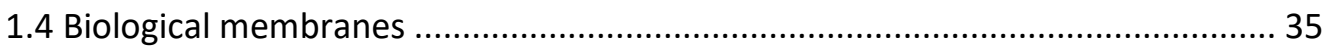

1.4.1 Structure of the membrane............................................................... 35

1.4.2 Lipid composition. ......................................................................... 35

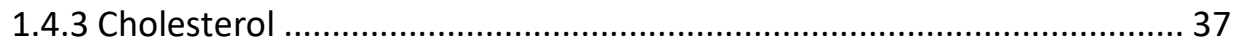


1.4.3.1 Structural importance of cholesterol in membranes................ 37

1.4.3.2 Cholesterol biosynthesis pathway .......................................... 38

1.4.4 Regulation of cholesterol biosynthesis ............................................. 41

1.4.4.1 Sterol regulatory-element binding proteins (SREBPs) .............. 41

1.4.4.2 Mechanism of activation of SREPBs ....................................... 41

1.4.5 Control of lipids biosynthesis ........................................................ 42

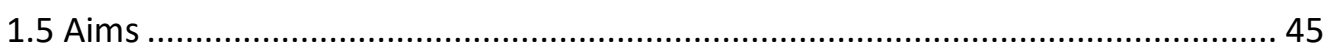

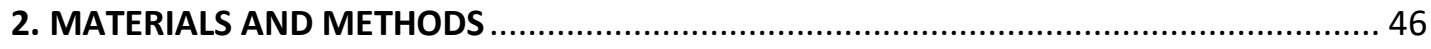

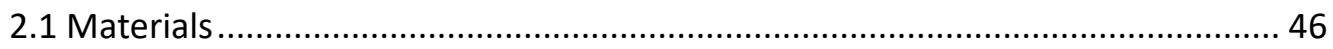

2.1.1 Consumables and Kits.................................................................. 46

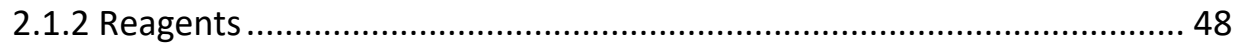

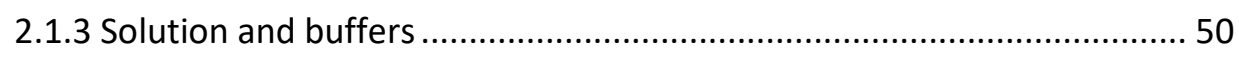

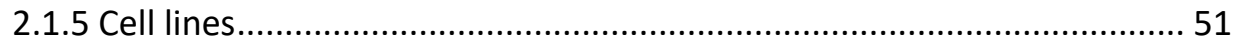

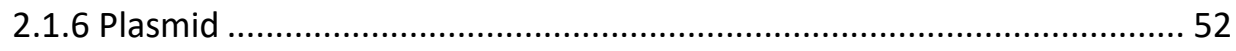

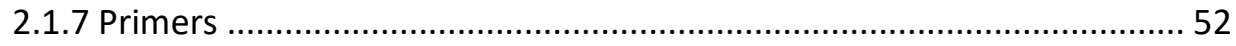

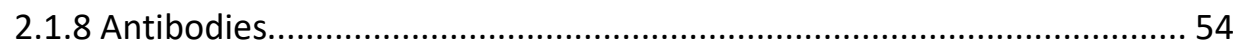

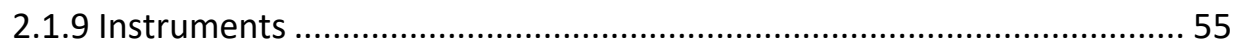

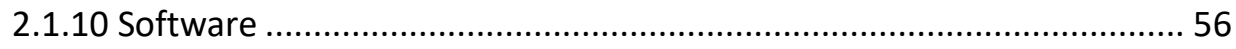

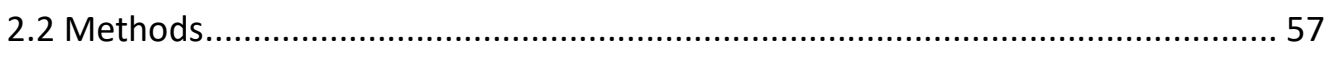

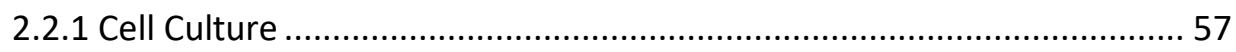

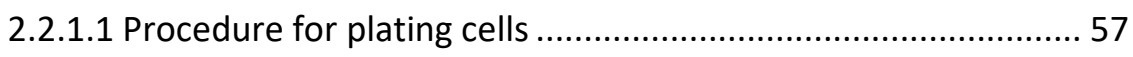

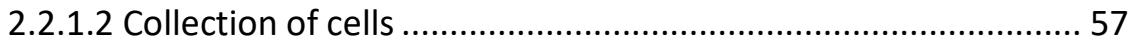

2.2.1.3 Mitochondrial isolation from cultured cells.............................. 57

2.2.1.4 Pharmacological treatments .................................................. 58

2.2.1.5 Generation of stable HeLa KDs ............................................. 58

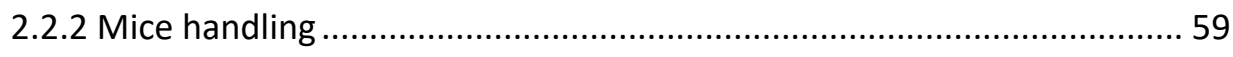

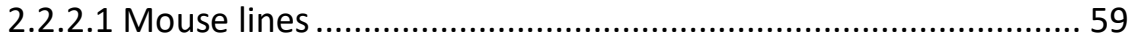

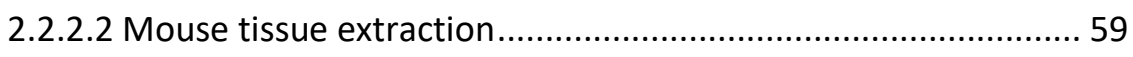

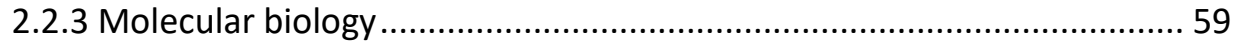

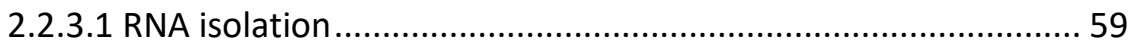




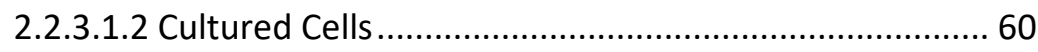

2.2.3.2 Determination of nucleic acid concentrations........................... 60

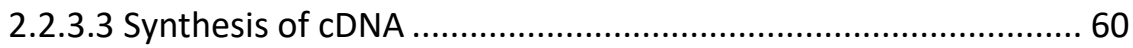

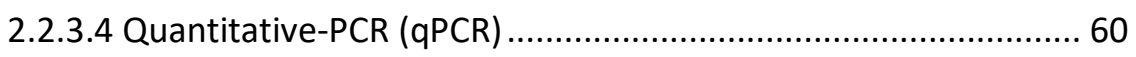

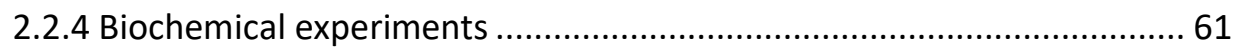

2.2.4.1 Preparation of protein samples from mouse............................ 61

2.2.4.1.1 Protein extraction from tissue powder........................ 61

2.2.4.1.2 Protein concentration determination of tissue

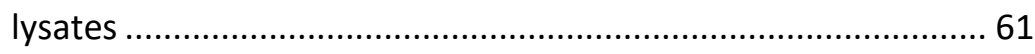

2.2.4.2 Preparation of proteins from cells ........................................ 61

2.2.4.2.1 Protein extraction from pellets................................... 61

2.2.4.2.2 Protein concentration determination of cell

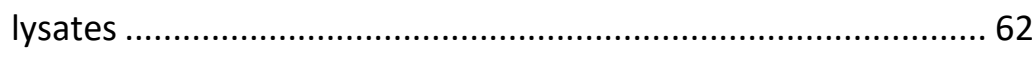

2.2.4.3 SDS-Polyacrylamide gel electrophoresis .................................. 62

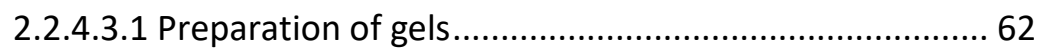

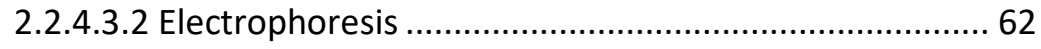

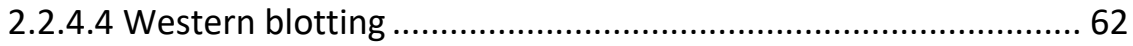

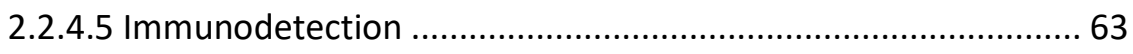

2.2.5 Cholesterol content measurement ….................................................. 63

2.2.5.1 Free cholesterol content measurement by Filipin ..................... 63

2.2.5.2 Protein concentration determination using

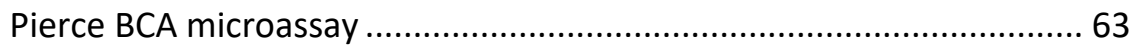

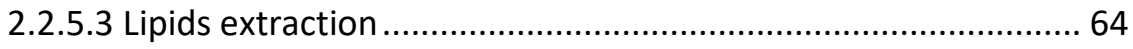

2.2.5.4 Cholesterol quantification using Amplex ${ }^{\circledast}$

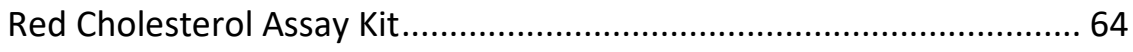

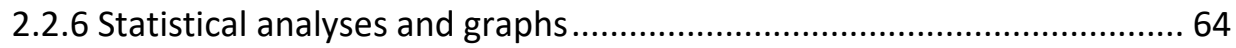

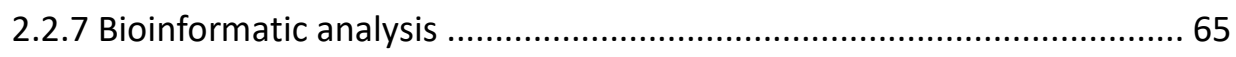

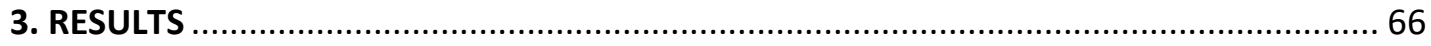

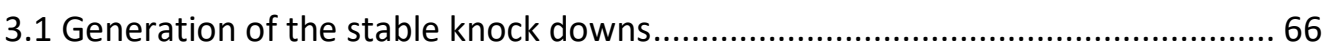


3.2 Validation of the data obtained from the analyses of the RNA Seq results 68

3.2.1 Mitochondrial dysfunction model 68

3.2.2 Models of lysosomal dysfunction 69

3.3 Regulation of cholesterol pathway by SREBP1 70

3.4 Cholesterol biosynthesis pathway is downregulated in

a mouse model of mitochondrial dysfunction. 71

3.5 Cholesterol biosynthesis is upregulated in three independent mouse models of lysosomal dysfunction 73

3.6 Cholesterol biosynthesis activity is downregulated in Ndufs 4 KO mice and upregulated in Gaa KO mice.

3.7 Pharmacological induction of mitochondrial or lysosome dysfunction 75

3.7.1 Mitochondrial respiratory chain inhibition does not affect the enhanced activity of cholesterol synthesis in lysosomal defects 75

3.7.2 Treatment with U18666A increases the transcript levels of main cholesterol biosynthesis enzymes in Ndufs4 KO MEFs. 76

3.8 Cholesterol: measurement and reduction 77

3.8.1 Measurement of free cholesterol content in HeLa model cells and Cts KO MEFs by filipin 77

3.8.2 Total cholesterol content is reduced in Ndufs4 KO MEFs and not affected in lysosomal dysfunction 78

3.8.3 Mitochondrial cholesterol is increased in Ndufs4 KO MEFs 79

3.9 Treatment with hydroxypropyl- $\beta$-cyclodextrin (HPCD) upregulates

the cholesterol biosynthesis in CtsB and L KO 80

3.10 Treatment with HPCD in Ndufs4 KO MEFs increased the transcript levels

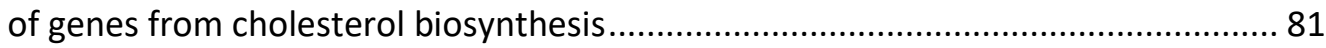

3.11. Determination of signaling pathways involved ............................................ 82

3.11.1 Blocking mTORC activity enhances the cholesterol biosynthesis pathway in CtsB KO MEFs.

3.11.2 Blocking mTORC activity does not affect the cholesterol biosynthesis pathway in Ndufs4 KO MEFs 
3.12 Upregulation of the cholesterol biosynthesis pathway in AMPK DKO MEFs ..... 85

3.13 Measurement of AMPK activity in several models of lysosomal dysfunction.... 87

3.14 Characterization of Lamp2 KO MEFs in the context of cholesterol transport.... 89

3.15 Comparison of AMPK activity between Ndufs4 KO MEFs and Lamp2 KO MEFs 91

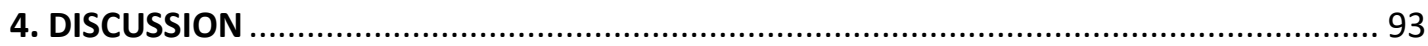

4.1 Validation of the data obtained from NGS analysis .......................................... 93

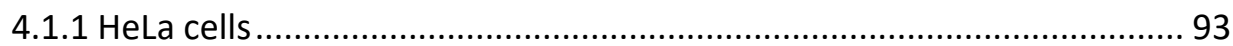

4.1.2 Mouse embryonic fibroblasts and mice ............................................. 95

4.1.3 Effect of lysosomal dysfunction in a model of mitochondrial dysfunction and vice versa .................................................. 96

4.2 Cholesterol: measurement and reduction .................................................... 96

4.2.1 Measurement of cholesterol content ................................................. 96

4.2.2Cholesterol extraction with hydroxypropyl- $\beta$-cyclodextrin (HPCD) ......... 98

4.3 Determination of signaling pathways involved ................................................ 98

4.3.1 Lipid pathway biosynthesis induced by mTORC .................................... 98

4.3.2 Lipid pathway biosynthesis repressed by AMPK .................................... 99

4.3.3 Regulation of HMGCR ..................................................................... 100

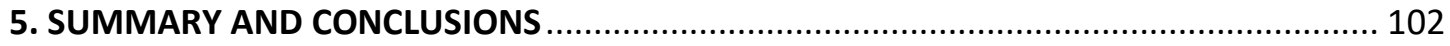

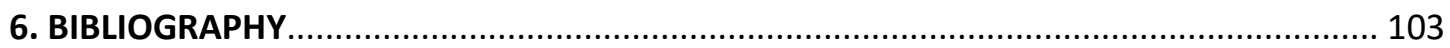

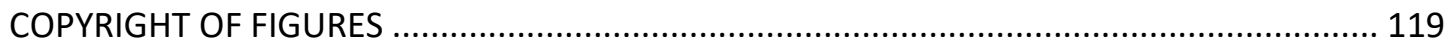

CURRICULUM VITAE 


\section{Abstract}

Mitochondria are known as the powerhouse of the cells. However, in recent years, it has become increasingly evident that mitochondria are involved in several signaling pathways. Lysosomes are membrane-bound organelles that receive and degrade macromolecules from the secretory, endocytic and autophagy pathways. Our lab has recently reported that mitochondrial respiratory chain deficiency inhibits lysosomal hydrolysis and that lysosomal lipid storage diseases are characterized by the repression of mitochondrial biogenesis; thereby showing interdependence between these organelles. However, little is known about the connection between the dysfunction of these organelles and lipid metabolism.

Here, we aimed to elucidate if the dysfunction in mitochondria or lysosomes yields any consequences on lipid metabolism, more specifically cholesterol biosynthesis, and to describe the underlying mechanism. We have employed cell lines with defects in each of these organelles and measured the effects on gene expression by RNA sequencing (RNA-Seq), followed by comprehensive multi-dimensional transcriptomics analysis, as well as by quantitative real-time PCR. Interestingly, changes in the expression of genes of the cholesterol synthesis pathway were among the significant consequences of both the mitochondrial and the lysosomal perturbations, but with opposite trends. While mitochondrial dysfunction results in the downregulation of the cholesterol biosynthesis, lysosomal dysfunction leads to its activation.

Analysis of the cholesterol content showed a significant decrease of unesterified (free) cholesterol level in cells with mitochondrial dysfunction. On the other hand, free cholesterol level was increased in the cells with lysosomal dysfunction. Mitochondrial cholesterol content was increased only in the cells with mitochondrial dysfunction.

To further investigate the origin of the opposite trends of cholesterol biosynthesis resulting from organelle dysfunction, we measured the activity of SREBP1, an established regulator of the cholesterol biosynthesis. We found a decrease in the SREBP1 activity in cells with mitochondrial dysfunction and its increase in the case of lysosomal dysfunction. The crucial role of AMPK in the regulation of the cholesterol biosynthesis pathway is evidenced by the changes in AMPK activity in the cells with organelle dysfunctions: while in the case of mitochondrial dysfunction AMPK showed increased activity, the trend was opposite in the cells with lysosomal dysfunction. Importantly, based on our results, the effect of organellar dysfunction on cholesterol biosynthesis depends on AMPK activity.

This work contributes to the understanding of the underlying mechanisms of pathologies associated with organelle dysfunction, such as lysosomal storage diseases or mitochondrial diseases, and the impact they may have on lipid homeostasis, such as the cholesterol biosynthesis. 


\section{List of abbreviations}

4-(2-hydroxyethyl)-1-piperazineethanesulfonic acid

HEPES

Acid alpha-glucosidase

GAA

Adenosine diphosphate

AMP

Adenosine triphosphate

ATP

AMP activated protein kinase

AMPK

Bovine serum albumin

BSA

Cathepsin

CTS

Coenzyme A

CoA

Complementary DNA

cDNA

Dimethyl sulfoxide

DMSO

Dulbecco's modified eagle's medium

DMEN

Flavin adenine dinucleotide

FAD

Inner mitochondrial membrane

IMM

Leigh syndrome

LS

Lysosomal storage diseases

LSDs

Mammalian Target of Rapamycin

mTOR

Nicotinamide adenine dinucleotide

$\mathrm{NADH}$

Outer mitochondrial membrane

OMM

Phosphate buffered saline

PBS

Polyvinylidene fluoride

PVDF

Quantitative real time PCR

qPCR

Reactive oxygen species

ROS

Short hairpin RNA

ShRNA

Sodium dodecyl sulfate

SDS

Tricarboxylic acid cycle

TCA 


\section{List of Figures}

FIGURE $1 \quad$ Schematic illustration of the mitochondrion $\quad 18$

FIGURE 2 Human mitochondrial genome 21

$\begin{array}{lll}\text { FIGURE } 3 & \text { Products of one turn of the citric acid cycle } & 24\end{array}$

$\begin{array}{lll}\text { FIGURE } 4 & \text { The functional features of the mitochondrial respiratory chain and } & 25 \\ \text { oxidative phosphorylation system }\end{array}$

FIGURE 5 Electron microscopy image of mouse embryonic fibroblast 29

FIGURE 6 The macroautophagy process

FIGURE 7 Model of the fluid mosaic $\quad 35$

FIGURE 8 Schematic representation of the three types of lipid in the membrane 36

FIGURE 9 2D structure of a cholesterol molecule 38

FIGURE 10 The mammalian cholesterol biosynthetic pathway 40

FIGURE 11 Model of regulation of SREBP in ER membranes 41

FIGURE 12 Transcriptome data analyses 66

FIGURE 13 Top 10 canonical pathways affected in each model 67

FIGURE $14 \quad$ Transcriptome data analyses $\quad 68$

FIGURE 15 Validation of RNA Seq results in HeLa UQCRC 1 KD 69

FIGURE 16 Validation of RNA Seq results in HeLa CTSB and GAA 70

FIGURE 17 UQCRC1 KD HeLa decreases the protein levels of active SREBP1 71

FIGURE 18 Downregulation of cholesterol synthesis in Ndufs4 KO MEFs

FIGURE 19 Upregulation of the synthesis of cholesterol Gaa KO MEFs 73

FIGURE $20 \quad \begin{aligned} & \text { Upregulation of the synthesis of cholesterol in two different models of } \\ & \text { lysosomal dysfunction }\end{aligned}$

FIGURE 21 Transcript levels of tissue samples $\quad 75$

FIGURE 22 Lysosomal dysfunction model cells treated with rotenone 76

FIGURE 23 Pharmacological induction of cholesterol biosynthesis pathway. 77

$\begin{array}{ll}\text { FIGURE } 24 & \text { Measurement of free cholesterol content in HeLa KDs and CtsB and CtsL } \\ \text { KO MEFs }\end{array}$

FIGURE 25 Measurement of cholesterol content by Amplex Red 79 
FIGURE 26 Measurement of mitochondrial cholesterol content by Amplex Red

FIGURE 27 Analysis of the transcript levels of cholesterol related genes in CtsB and CtsL KO MEFs treated with HPCD

FIGURE 28 Analysis of the transcript levels of cholesterol-related genes in Ndufs4 MEFs treated with HPCD

FIGURE 29 Treatment with Torin increases the cholesterol biosynthesis pathway in CtsB KO MEFs

FIGURE 30 Treatment with Torin does not affect the cholesterol biosynthesis pathway in Ndufs4 KO MEFs

FIGURE 31 AMPK DKO MEFs cannot restore normal transcript levels of cholesterol biosynthesis genes under pharmacological co-treatment

FIGURE 32 Active SREPB1 protein levels are not affected by pharmacological treatments AMPK DKO MEFs

FIGURE 33 AMPK activity is not decreased in all the studied models of lysosomal dysfunction

FIGURE 34 Upregulation of transcript levels involved in the cholesterol biosynthesis pathway in Lamp2 KO MEFs

FIGURE 35 Evaluation of the AMPK activity through the phosphorylation AMPKa Thr 172

FIGURE 36 Evaluation of cholesterol pathway activation by SREBP1 and PHMGCR in Lamp2 KO MEFs

FIGURE 37 Comparison of Ndufs4 KO and Lamp2 KO MEFs in the activation of the cholesterol biosynthesis pathway 


\section{List of Tables}

TABLE 1 Lipid composition of subcellular fraction of rat liver 36

TABLE 2 List of consumables and kits 46

TABLE 3 List of reagents 48

TABLE $4 \quad$ List of solution and buffers $\quad 50$

TABLE 5 Cell culture materials 51

TABLE 6 Plasmids used in this thesis 52

TABLE 7 Human primers

TABLE 8 Mouse primers 53

$\begin{array}{lll}\text { TABLE } 9 & \text { Primary antibodies for immunoblotting } & 54\end{array}$

TABLE 10 Secondary antibodies for immunoblotting 55

TABLE 11 List of instruments and equipment 55 


\section{INTRODUCTION}

\subsection{Cellular biology}

Cells are the fundamental units of life, from which we must look for answers to the questions of what life is and how it works. A typical structure of all cells is that each of them is enclosed by a plasma membrane. This membrane is essential for the cell because it acts as a selective barrier allowing the cell to concentrate nutrients obtained from the environment and keep within its interior the newly synthesized macromolecules while excreting its waste products. As such, the cell acquires its integrity as a coordinated chemical system thanks to the plasma membrane. Biological membranes consist of a bilayer formed by amphiphilic molecules, mostly lipid molecules, and proteins.

Today, cells are grouped into three major divisions or domains: bacteria, archaea, and eukaryotes (Alberts, 2014). Whereas the cytological properties of bacteria and archaea are relatively simple, eukaryotes are characterized by a high degree of cellular complexity (Spang et al., 2015). Bacteria and archaea are unicellular entities, formed by prokaryotic cells. These cells have in common a lack of membrane-bound organelles or other internal membrane-bound structure.

Eukaryotic cells, by definition, have an internal compartment called nucleus that divides the DNA from the cytoplasm. The double-layer membrane that surrounds the nucleus is denominated nuclear envelope. Another critical component of eukaryotic cells is the presence of organelles, intracellular membrane-bound structures inside the cell usually specialized for a particular function (Mullock and Luzio, 1976).

The mitochondrion is an exceptionally complex organelle. Due to its prokaryotic origin almost 1.5 billion years ago (Dyall, 2004), this organelle has two membranes, inner and outer mitochondria membrane, and its small genome. Although the mitochondria are well known to be essential for bioenergetics, the complexity of mitochondrial metabolism has been reported in emerging research, associating it to diverse functions (Spinelli and Haigis, 2018).

Lysosomes, or vacuoles as they are referred to as in fungi and plants, are organelles which function as the significant degradative compartment in the cell. Lysosomal $\mathrm{pH}$ is maintained between $4.6-5.0$ actively and mainly by a proton-pumping vacuolar ATPase (VATPase) although there are more transporters that contribute to lowering the $\mathrm{pH}$, such as the SLC36 family (amino acid transporter) (Thwaites and Anderson, 2011) and CIC-7 (chloride antiporter) (Scott and Gruenberg, 2011). The low $\mathrm{pH}$ allows the acidic hydrolases to be able to degrade the macromolecules delivered to the lysosome (Lübke et al., 2009). They are associated with different degrative pathways, such as endocytosis where lysosomes are the terminal organelle 
and autophagy, the process in which the intracellular material is isolated and digested (Luzio et al., 2007) (Olson and Joyce, 2015).

Lipids play an essential role in different cellular processes and are one of the primary class of biological membranes. Different thousands of lipids are synthesized in mammalian cells, as well as hundreds of proteins to synthesize, metabolize, transport them (Muro et al., 2014). However, less is known about lipids than any other different kind of molecules such as proteins. Even today when lipids can be used in laboratories for signaling, cell structure, and other functions the knowledge about their function in lipid droplets, lipoproteins, and biological membranes is still rudimentary (Merrill, 2013). This project focuses on changes in the cholesterol synthesis as a consequence in the dysfunction of two different organelles, mitochondria and lysosomes. 


\subsection{Mitochondrial biology}

The most common phrase that precedes an introduction to mitochondria is: "mitochondria, the organelles that act as cellular powerhouses" (de Bock and Thorne, 2016). However, nowadays, it is accepted that mitochondria are multifaceted organelles involved in several essential processes for the cell.

Mitochondria have a role in critical in the cell being part of processes such as autophagy, cell proliferation, differentiation, cellular immunity, and apoptosis (Nunnari and Suomalainen, 2012; Raimundo, 2014). Several diseases have been associated with mitochondrial dysfunction. Genetic disorders, characterized by defects in the mitochondrial system, are a group of pathologies denominated mitochondrial diseases. These diseases are mainly caused by mutations in genes that encode proteins involved in the function or structure of the mitochondria. The mutations can be present in genes encoded in the mitochondrial DNA (mtDNA) or nuclear DNA (nDNA) (Gorman et al., 2016). Mitochondrial diseases present a different phenotype in patients, affecting several different organs, and generating a multisystem presentation (Lightowlers et al., 2015).

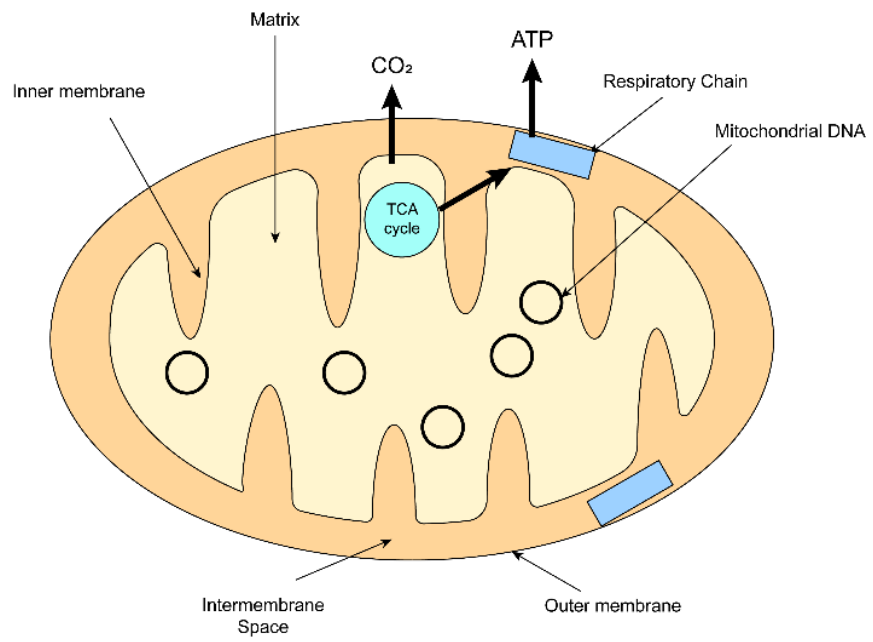

Figure 1. Schematic illustration of the mitochondrion. The mitochondrial structure is defined by the presence of outer and inner membranes, with cristae in the inner membrane and the matrix where the mitochondrial DNA is present.

\subsubsection{Structure of the mitochondria}

In 1898, the name mitochondrion was introduced. The origin of the name is from the Greek words, "mitos" (thread) and "chondros" (granule) (Ernster and Schatz, 1981). Mitochondria basic structure is characterized by an outer (OMM) and inner membranes (IMM). These membranes enclose the intermembrane space (IMS) and matrix compartments, respectively form 
mitochondria. Mitochondria is the only organelle in mammalian cells that carry its circular genome, mitochondrial DNA. Mitochondrial DNA is organized into nucleoids in the mitochondrial matrix (Nunnari and Suomalainen, 2012) (Fig. 1).

The outer membrane allows the traffic of small molecules and ions as large as 5kDa. However, in this membrane, an important channel is located, the voltage-dependent anion channel (VDAC). The primary function of this channel is as a gatekeeper for the exit and entry of mitochondrial metabolites, regulating the cross-talk between the rest of the cell and mitochondria (ShoshanBarmatz et al., 2010).

The inner membrane has a larger surface area compared to the outer membrane. One of the main characteristics of the IMM is the presence of features referred to as cristae (literally, crests). Initially, cristae were thought to be simple infoldings of the inner membrane (Frey and Mannella, 2000). However, during the 1990s, Mannella et al., employing electron tomography 3D reconstruction, showed that the cristae are a bag-like structure (Mannella, 2006). This conformation suggests that cristae are specialized compartments for limiting the diffusion of molecules necessary for the respiratory chain (Cogliati et al., 2016). The cristae membrane is enriched in proteins involved in protein synthesis and translocation, in iron-sulfur biogenesis, and mitochondrial nucleoid maintenance (Vogel et al., 2006), and houses assembled respiratory chain complexes and supercomplexes (Cogliati et al., 2013). The density of proteins in this membrane is approximate of $75 \%$ by weight and the lipid content of $25 \%$, making the cristae membrane one of the densest protein membranes in all biological membranes (Pernas and Scorrano, 2016).

The space delimitated by the membranes is the IMS and the matrix. Due to the $\mathrm{pH}$ gradient $(\Delta \mathrm{pH})$ between the matrix and the IMS, protons are pumped from the matrix to the space formed inside of the cristae (cristae space). The cristae space is needed for the synthesis of ATP in the mitochondria (Friedman and Nunnari, 2014). Most of the metabolic processes carried out by the mitochondria take place in the matrix, such as Krebs cycle, one-carbon metabolism, and fatty acid oxidation(Ducker and Rabinowitz, 2017; Röhrig and Schulze, 2016).

When observed the mitochondria in live cells, it can be seen that their morphologies are not static. The combined actions of fusion, fission, and motility modulate continuously the mitochondrial shape (Youle and van der Bliek, 2012). This structure formed is denominated mitochondrial network, which is very dynamic. Typical conformations are fluctuating between a tubular continuum, and a fragmented state(Benard et al., 2007). Changes in the mitochondrial network play a crucial role in apoptosis, $\mathrm{Ca} 2+$ transfer, and mitochondrial quality control (Rambold et al., 2011).

Large guanosine triphosphatases (GTPases) mediate the process of mitochondrial fission and fusion (Hoppins et al., 2007). The fission process is mediated by a cytosolic dynamin family member (dynamin-related protein 1 (DRP1)). DRP1 constricts to sever both inner and outer 
membranes when is recruited from the cytosol to form spirals around mitochondria. In mammals, four mitochondrially localized adaptor proteins play essential roles in the recruitment of DRP1 to mitochondria: mitochondrial fission factor (MFF); mitochondrial dynamics proteins of 49 kDa and 51 kDa (MiD49 and MiD51); and fission 1 (FIS1), (Pernas and Scorrano, 2016). After GTP hydrolysis, DRP1 helix constricts, and the mitochondrion is divided.

Fusion is a process that requires that two mitochondria get close enough to contact for the initiation (Meeusen et al., 2004). Once this contact is initiated, the dynamin-related OMM proteins, Mitofusin 1 and Mitofusin 2 (MFN1 and MFN2) form in trans, homotypic or heterotypic complexes (Koshiba et al., 2004) between neighboring mitochondria mediating the OMM fusion. Nevertheless, recently, MFN2 was described to be necessary for the ER-mitochondrial contact sites (Filadi et al., 2018). Next, OPA1 mediates the IMM fusion in a process dependent on inner membrane potential (Chan, 2012). The fusion of a 'less fit' mitochondrion with a 'healthy' mitochondrion is proposed to be part of a mechanism to maintain a healthy mitochondrial population. The role of the fusion process is to maintain genetic and biochemical homogeneity by permitting the dilution of superoxide, mutant DNA, and repolarization of the membranes (Pernas and Scorrano, 2016). In this way, mitochondrial fusion is a necessary process, which compensates for mutations in mitochondrial DNA, thereby allowing mitochondria to share components as long as the mutation load remains below thresholds of $80 \%$ to $90 \%$ per cell (Nakada et al., 2001).

\subsubsection{Mitochondrial DNA}

The mtDNA is a gene-dense, double-stranded DNA (dsDNA) molecule of $16.6 \mathrm{~kb}$, which encodes 11 messenger RNAs (mRNAs) (translated to 13 proteins), 2 ribosomal RNAs (rRNAs) (12S and 16S rRNA), and 22 tRNAs (Gustafsson et al., 2016)(Fig. 2). The OXPHOS system that consists of approximately 100 proteins, 13 subunits are encoded in the mitochondrial DNA. However, if the expression of the subunit encoded in the mtDNA is not optimal, OXPHOS collapse (Larsson et al., 1998). On the contrary, to nuclear DNA, mtDNA consists of a circular structure and lacking intronexon structure. The replication of mtDNA is regulated by the displacement loop (D loop), a single non-coding region. Even though several copies of mtDNA are present within each cell, the total amount of mtDNA can vary between hundreds to thousands of copies according to the cell type (Gorman et al., 2016). 


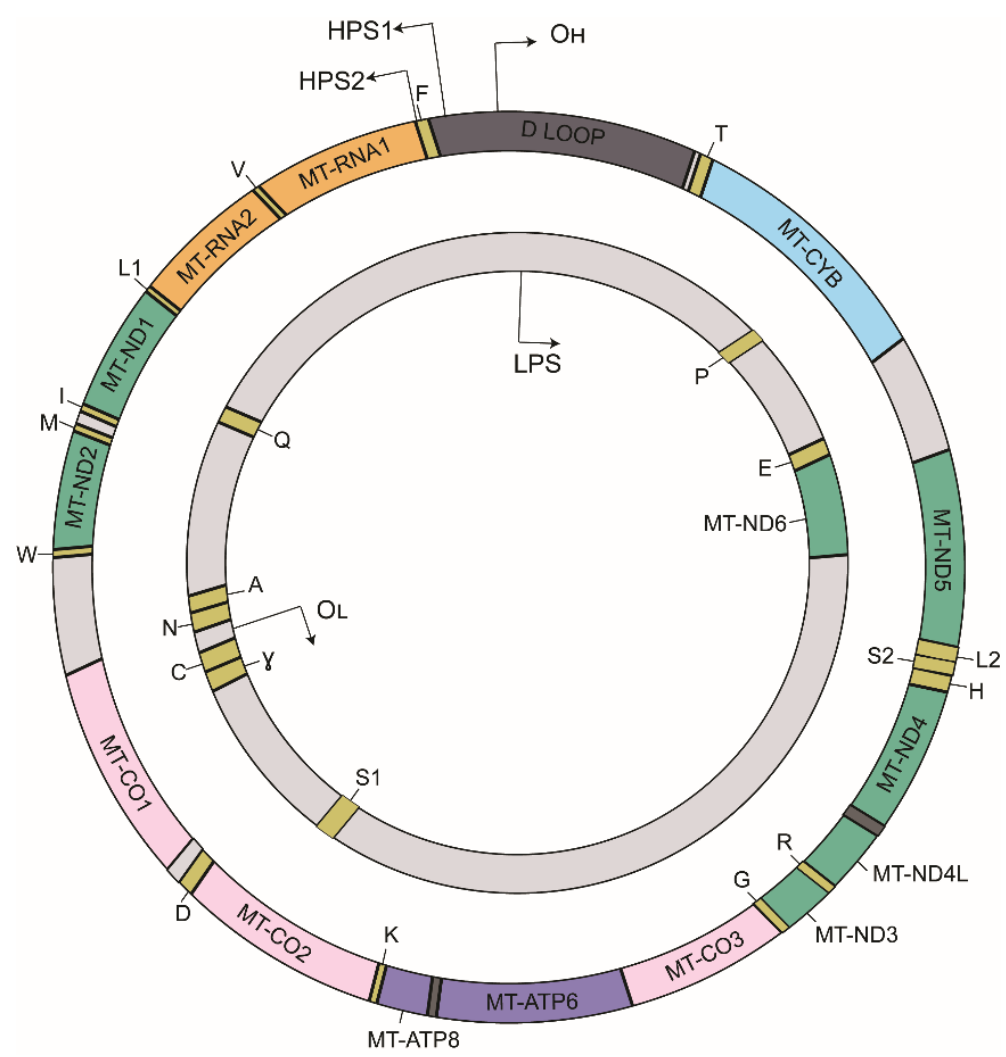

Figure 2. Human mitochondrial genome. Mitochondria has his genome, in human cells, consist in a circular doublestranded DNA molecule of $16.6 \mathrm{~kb}$. The outer circle corresponds to the H-strand carrying most of the genes; the inner circle is the L-strand. It includes a 1.1-kb non-coding region. Adapted from (Gorman et al., 2016).

Recently, it has been discovered in mammalians cells by super-resolution microscopy experiment the structure of the mitochondrial DNA nucleoid. The structure of the mtDNA consists of compacted DNA into nucleoid structures with a mean size of approximately $100 \mathrm{~nm}$ (Brown et al., 2011). Most nucleoids are formed by just one single copy of mtDNA. The main packing factor, considered the histone-like protein of mtDNA, is the mitochondrial transcription factor $A$ (TFAM)(Kukat et al., 2011). Several molecules of TFAM binding one mtDNA molecule hence regulating the compaction of the nucleoid (Picca and Lezza, 2015). For example, Jakobs and colleagues (Kukat et al., 2011) reported approximately 1000 TFAM molecules per mtDNA molecule in human fibroblasts.

The two strands of mtDNA, which were identified initially by density ultracentrifugation, are named the heavy $(\mathrm{H})$ and light $(\mathrm{L})$ strands (Clayton, 1982). Leading promoter for the transcription of each strand denominated the L strand promoter (LSP), and the $\mathrm{H}$ strand promoter (HSP) are present in the mammalian mtDNA. Three proteins are essential for the basal transcription initiation: RNA Polymerase Mitochondrial (POLRMT), TFAM, and the mitochondrial transcription factor B2 (TFB2M) (Hällberg and Larsson, 2014). 
The replication and repair of mtDNA are done by mtDNA polymerase $\gamma$ (POLG). This protein works in conjunction with several additional replisome components including topoisomerase, Twinkle, a mitochondrial RNA polymerase (mtRNAP), RNaseH1, mitochondrial single-stranded DNAbinding protein ( $\mathrm{mtSSB}$ ), and mitochondrial DNA ligase III (Young and Copeland, 2016). Similar to the replication of nuclear DNA, the role of primase for the replication is done by mitochondrial RNA polymerase (mtRNAP) (Picca and Lezza, 2015).

One of the first models described for the mtDNA replication, displacement loop model (stranddisplacement model), was proposed in 1972 (Robberson and Clayton, 1972). This model had been widely accepted to be the only mechanism of mammalian mtDNA replication. By the year 2000, a new model was proposed; in this case, mtDNA replication intermediates (RIs) were noticed that had properties of conventional, coupled leading- and lagging-strand DNA synthesis in tissue and cultured cells of human and mouse (Holt et al., 2000). In the years following, it was suggested that mtDNA replication could start at either one of the two sites. One of these sites is the OL site, and another one around approximately $13 \mathrm{~Kb}$ nucleotides, inside of the ND5 gene of the L-strand. DNA synthesis spreads in the leading strand, simultaneously incorporating RNA throughout the lagging strand (RITOLS)(Yasukawa and Kang, 2018).

\subsubsection{Mitochondria in metabolism}

Mitochondria have several vital roles in cellular metabolism. They are an essential producer of building blocks for biosynthetic pathways. For example: synthesis of nucleotides, in which the one-carbon metabolic pathway is involved; amino acids synthesis including glutamate, alanine, proline, and aspartate (Ducker and Rabinowitz, 2017); production of acetyl-CoA for fatty acids synthesis (Hatzivassiliou et al., 2005); and glucose synthesis since the initial enzyme for gluconeogenesis, pyruvate carboxylase, is located in the mitochondria (Bahl et al., 1997).

However, one of the most studied roles of mitochondria is the production of energy. Mitochondria integrates the fuel metabolism to generate energy in the form of ATP by oxidizing, fatty acids (Röhrig and Schulze, 2016), amino acids to give electrons onto the carriers NADH and FADH2 (DeBerardinis and Cheng, 2010), and pyruvate (derived from lactate or glucose) (Heiden et al., 2009).

\subsubsection{Glucose metabolism}

Glucose has an essential role in the metabolism of organisms. The importance of glucose in energy production is based on it relatively rich in potential energy, making it a proper fuel. The oxidation of glucose to water and carbon dioxide generates $-2,840 \mathrm{~kJ} / \mathrm{mol}$ (Boyle, 2005). This reaction starts in the cytoplasm with a process called glycolysis, where glucose is oxidized to a 
three-carbon compound, pyruvate. The next two steps for the oxidation of pyruvate take place in the mitochondria, via the TCA cycle and the respiratory chain.

\subsection{Glycolysis}

The metabolic pathway that converts glucose into pyruvate is called glycolysis. These set of reactions take place in the cytoplasm and generates adenosine triphosphate (ATP). The glycolysis pathway comprises ten steps of chemical reactions, each catalyzed by a specific enzyme (Li et al., 2015). During this process, three types of chemical reactions are particularly notable: (1) degradation of glucose to produce pyruvate, (2) production of ATP by phosphorylation of ADP to ATP, and (3) production of NADH. The final product of glycolysis, pyruvate, is oxidated to acetylCoA by process of oxidative decarboxylation. The acetyl group is then fully oxidized to $\mathrm{CO} 2$ by the TCA cycle (Boyle, 2005).

\subsection{Citric acid cycle}

The citric acid cycle (also known as tricarboxylic acid (TCA) cycle or the Krebs cycle) was discovered by Hans Adolf Krebs in 1937. All the aerobic processes in animal tissue obtain energy from this primary metabolic pathway. The enzymes involved in the citric acid cycle are located in the mitochondria (Nazaret et al., 2009). Acetyl-CoA could be obtained from glucose via glycolysis or from fatty acids that have gone through $\beta$-oxidation. During the TCA, citrate is oxidated into isocitrate by aconitase. Isocitrate dehydrogenase by a decarboxylation reaction converts isocitrate to $\alpha$-ketoglutarate $(\alpha K G)$. In this way, each new turn in the cycle provides a significant new amount of cellular ATP (Akram, 2014). The cycle only generates one molecule of GTP per cycle (during the conversion of succinyl-CoA to succinate). However, nicotinamide adenine dinucleotide (NAD+) and flavin adenine dinucleotide (FAD) is reduced to obtain NADH and FADH2 during the cycle. These molecules provide a massive flow of electrons into OXPHOS (Fig. 3). 


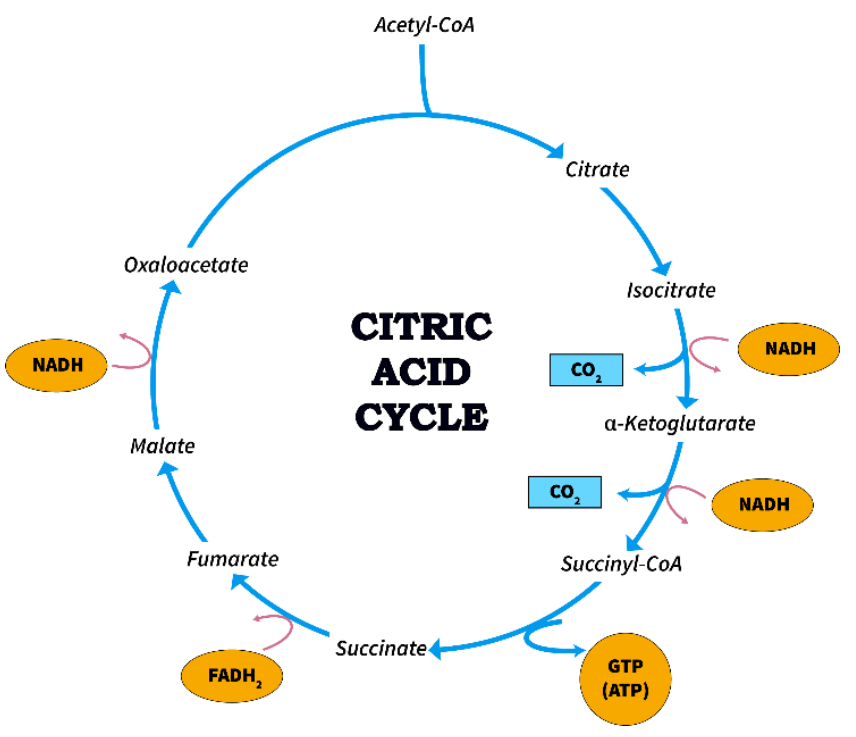

Figure 3. Products of one turn of the citric acid cycle. At each turn of the cycle, two $\mathrm{CO} 2$ are released in oxidative decarboxylation reactions. The energy production is obtained from the three NADH, one FADH2, and one GTP (or ATP). Even though the schematic representation of the cycle shows the reaction in only one way, the reactions are reversible. Figure adapted from (Boyle, 2005)

\subsubsection{Respiratory chain}

Most of the cellular ATP is produced in the mitochondria, more specifically in the cristae that contain the ATP synthase enzyme. In the same membrane is located the large protein complexes of the respiratory chain (electron transport chain). 

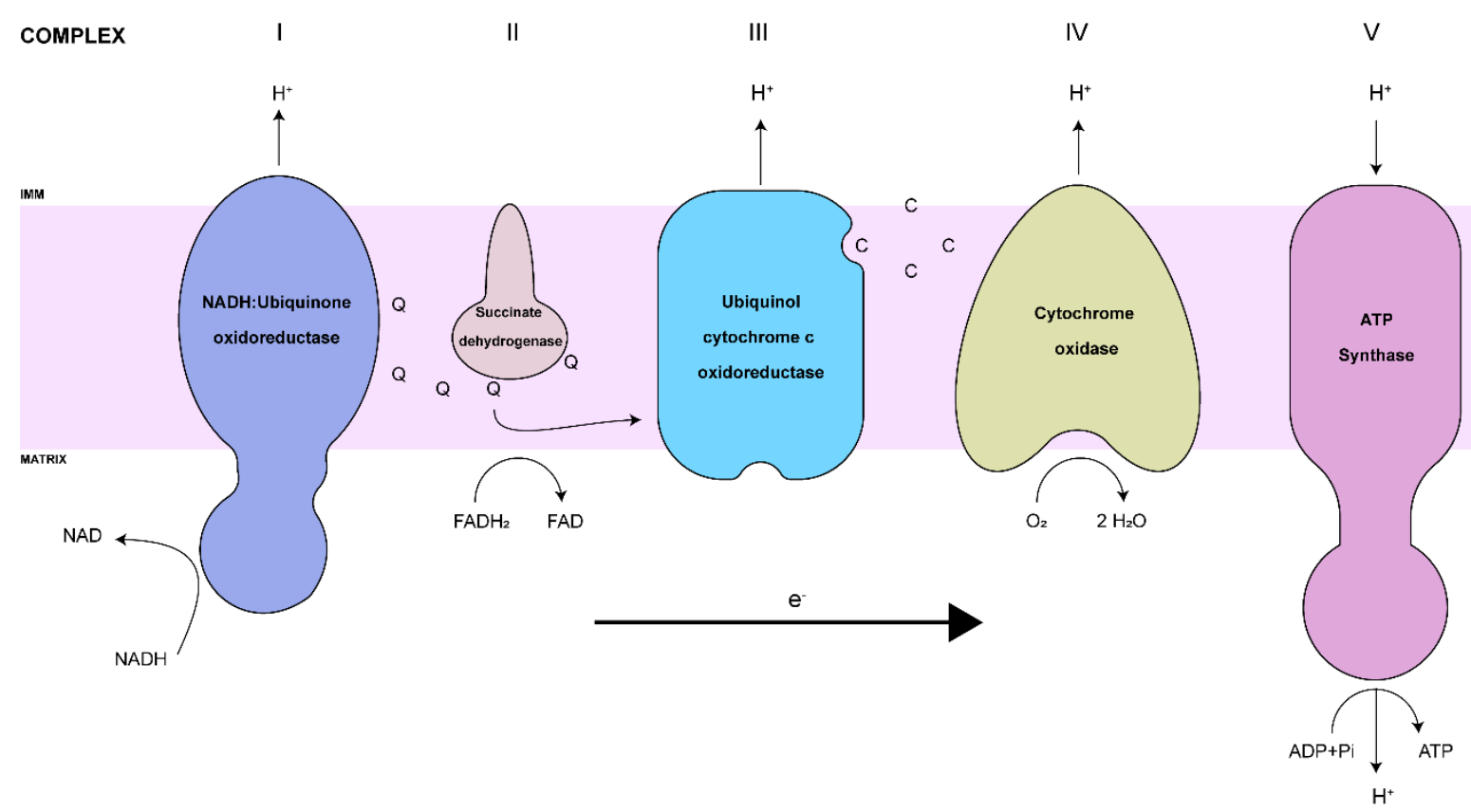

Figure 4. The functional features of the mitochondrial respiratory chain and oxidative phosphorylation system. The black arrow represents the transfer of electrons produced by oxidation of NADH and succinate by complex I and II, respectively. Q: ubiquinone, C: Cytochrome c. Figure adapted from (Suomalainen and Battersby, 2018)

During the citric acid cycle, a large amount of $\mathrm{NADH}$ and $\mathrm{FADH} 2$ are generated. Nevertheless, these molecules can be generated in other metabolic pathways and are used in the respiratory chain. NADH is oxidized by Complex $\mathrm{I}$; in this process, electrons are transported through flavin mononucleotide (FMN), and multiple iron-sulfur (Fe-S) centers present in Complex I till they are transferred to coenzyme Q10 (CoQ10). Complex II and the FADH2 generated by $\beta$-oxidation donate electrons to CoQ10. This coenzyme carries the electrons to Complex III. Once in Complex III, electrons pass from the cytochrome $b$ to cytochrome $c 1$, and at the end to Fe-S components. Cytochrome $\mathrm{c}(\mathrm{Cyt} \mathrm{C})$ is responsible for the transference of electrons from Complex III to Complex IV. Inside Complex IV, the electrons are transferred from the copper centers and cytochromes to $\mathrm{O} 2$ molecules to generate $\mathrm{H} 2 \mathrm{O}$ finally. The energy obtained from the respiratory chain is used to actively pump protons through Complexes I, III, and, IV; from the mitochondrial matrix to the intra-cristae space. Finally, ATP is synthesized in Complex $V$ as a consequence of the resulting electrochemical gradient because the gradient forces protons to move back through a proton channel in this complex (Alberts, 2014; Boyle, 2005; Chance and Williams, 2006; El-Hattab and Scaglia, 2016) (Fig. 4).

\subsubsection{Mitochondrial communication}


The production of energy by the respiratory chain and different metabolites synthesized in the TCA cycle gives to mitochondria a crucial role in diverse biological processes. Such as cellular proliferation, differentiation, and adaptation to stress (Chandel, 2015). Mitochondria can alter the expression of nuclear genes through signals, thus modifying the function of the cell and reprogramming the metabolism. This kind of response is present in all organism, but the nature of the pathways involve, and the regulation varies. Depending on the trigger, the different pathways can be classified into $\mathrm{Ca}+$ dependent, energetic stress, and reactive oxygen species (ROS) stress responses (Jazwinski, 2013).

In mammals, alterations in mitochondrial metabolism activate several pathways. For example, a reduction in ATP synthesis activates AMP-activated protein kinase (AMPK), which activates the mitochondrial biogenesis (Garcia-Roves et al., 2008). AMPK activated triggers changes in the expression of genes of mitochondrial proteins (Kelly, 2004). Additionally, the mitochondrial quality control system is activated by AMPK (Egan et al., 2011).

Reactive oxygen species (ROS) are mostly produced during the process of oxidative phosphorylation. Complexes I and III can prematurely reduce oxygen, generating superoxide anion (O2 •-) (Murphy, 2009). Additionally, $\mathrm{H} 2 \mathrm{O} 2$ is produced from the dismutation of superoxide (Wong et al., 2017). The mitochondrial ROS was always considered as a mere result of the inefficiencies of the OXPHOS. Nevertheless, it has emerged a critical role for mitochondrial ROS in the diffusion of cellular signaling pathways (Hamanaka and Chandel, 2010). Recent evidence suggests, for example, a role of $\mathrm{H} 2 \mathrm{O} 2$ emitted from mitochondria during hypoxia. Since $\mathrm{H} 2 \mathrm{O} 2$ can induce hypoxia-inducible transcription factors (HIFs), it has been identified as a central upstream regulator of many processes related to hypoxia (Waypa et al., 2006). Additionally, cellular survival and proliferation in cancer cells were reported due to the activation of NF-KB, activated by mitochondrial ROS (Formentini et al., 2012). Furthermore, the mitochondrial biogenesis and expression of OXPHOS genes are induced by ROS via JNK-PGC1 $\alpha$ signaling (Chae et al., 2013).

Another essential role of mitochondria is the regulation of intracellular calcium levels (Rizzuto et al., 2012). Under a condition in which the mitochondrial DNA is damaged or malfunction of electron transport chain complexes, it triggers the loss of membrane potential and the further release of $\mathrm{Ca} 2+$ into the cytoplasm (Amuthan et al., 2002).

Changes in the mitochondrial Ca2+ uptake is a significant cause of cell death, for this process is required the mitochondrial permeability transition pore (mPTP). During the formation of the MPTP, a nonspecific high-conductance channel is formed in the inner membrane allowing the movement of solutes up to 1 KDa unrestrictedly (Brenner and Moulin, 2012). This process generates a collapse of the membrane potential, mitochondrial swelling because of the osmotic influx of water, and ATP hydrolysis in Complex V(Bhosale et al., 2015). In the case of the rupture 
of the outer membrane due to the swelling several pro-apoptotic factors are released, such as cytochrome c (Bernardi et al., 2015; Bonora et al., 2015; Chinopoulos and Szabadkai, 2014).

\subsubsection{Mitochondria in pathology}

Mitochondrial diseases are characterized by a dysfunction in mitochondrial function generated by genetic disorders. These disorders are produced by mutations in mitochondrial or nuclear DNA, making the mitochondrial diseases a complex pathology (Nunnari and Suomalainen, 2012). For this reason, the observed phenotype is different in each patient. These diseases are reported to occur at any age and manifesting a variable range of clinical symptoms. They can affect any tissue or organ; in most of the cases, multiple systems are affected. The most common organs affected are reliant on aerobic metabolism (McFarland et al., 2010). Currently, mitochondrial disorders cannot be cured but can be treated. Most of the existing treatments are directed to boost mitochondrial biogenesis such as administration of bezafibrate (an agonist of PPAR), activation of AMPK by AICAR( 5-aminoimidazole-4- carboxamide ribonucleotide) or a ketogenic diet (Suomalainen and Battersby, 2018). These treatments increase mitochondria biogenesis, lipid oxidation, and in mitochondria myopathy, have been shown to improve muscle metabolism (Viscomi et al., 2011).

Mitochondrial protein synthesis machinery or subunits of the respiratory chain are the most commonly affected by mutations producing the pathologies. There are three types of mutations in the mtDNA causing mitochondrial diseases: the one that affects the protein synthesis (for example, mutations in mitochondrial tRNA or rRNA), affecting structural proteins for the mitochondria (for example, mitochondrial mRNA mutations) or rearrangements in the mitochondrial DNA (for example, sporadic, single, large-scale mtDNA deletions) (Gorman et al., 2015).

The most well-characterized childhood mitochondrial disorder is Leigh syndrome, affecting 1 in 40000 newborns in the USA (Cherezov et al., 2013). The pathology of has a significant variation between patients according to the age. Most commonly, patients present neurological symptoms such as ataxia, regression, and developmental delay. Although the symptoms can be multisystemic affecting the heart, liver, digestive system, and renal system (Lake et al., 2016). More than 75 genes mutations are associated with Leigh syndrome, and most of the mutated genes are components of the mitochondrial respiratory chain (Gorman et al., 2016). Nevertheless, the most frequent mutation associated to this syndrome is a mutation in the complex I; specifically, in NADH dehydrogenase (ubiquinone) Fe-sulfur protein 4 (NDUFS4) is the most frequent cause (Lake et al., 2016). For this reason, the Ndufs4 KO mice will be used as a model of mitochondrial dysfunction in this study 


\subsection{Lysosomal biology}

In the 1950s, Christian de Duve experimented with tissue fractionation, aiming to analyze the intracellular distribution of rat liver enzymes (de Duve et al., 1955). Upon realizing that five acid hydrolase enzymes were located in the same membrane-limited structure, it was suggested that these organelles were involved in intracellular digestion and hence named lysosomes (Greek for "digestive body") (de Duve, 2005).

During the observation of lysosomes with electron microscopy, it was determined that up to $5 \%$ of the intracellular volume correspond to lysosomes and that the morphology and size vary according to cell type. Additionally, it is observed the presence of electron-dense deposits (Holtzman, 1989). These organelles contain about 60 different degradative acidic enzymes. The enzymes digest different macromolecules such as DNA, RNA, proteins, polysaccharides, and lipids (Kolter and Sandhoff, 2005). All the lysosomal enzymes are acid hydrolases. In case of the lysosomal membrane brakes, the enzymes would be unable to degrade the cytoplasmic content protecting the cell from digestion. Mutation of the hydrolytic enzymes produces more than 30 different human genetic diseases. All these diseases are characterized by the presence of accumulated undigested materials inside of the lysosome, and because of that, they are called lysosomal storage diseases (LSD) (Cooper, 2000).

Few years after lysosomes were discovered, they were associated as a critical component of the two major degradative processes in the cell: endocytosis and autophagy. In the endocytosis, the lysosomes are the terminal degradative compartment, and in autophagy, the intracellular is degrade inside of the lysosomes (de Duve, 1983). In the last years, it has become clear that the lysosome does not merely function as a recycling center. This organelle is gaining prominence as a signaling hub for nutrients, with the v-ATPase playing an important role in sensing amino acid availability (Carroll and Dunlop, 2017).

\subsubsection{Structure of the lysosome}

Lysosomes are typically spherical with diameter between $100 \mathrm{~nm}$ and $500 \mathrm{~nm}$. At the ultrastructural level, multilamellar structures have been described inside of the lysosomes. These structures are formed as a result of the partial degradation vesicle membrane that has been delivered to the lysosome (Kolter and Sandhoff, 2010). The lysosomal membrane consists of a single membrane, in which several integral membrane proteins necessary for functions such as the lysosomal structural integrity (Fig. 5). The limiting outer membrane is composed of a phospholipid bilayer of 7-10 $\mathrm{nm}$ that is decorated with transmembrane proteins. Lysosomeassociated membrane proteins (LAMP)1 and LAMP2 are the most abundant of proteins in the membrane, which together constitute about $80 \%$ of the membrane proteins (Saftig and 
Klumperman, 2009). LAMP proteins and others, such as lysosomal integral membrane protein 2 (LIMP2) and CD63, are heavily glycosylated on their luminal side and form the glycocalyx. This barrier protects the membrane from the action of the enzymes inside the lysosomal lumen (Saftig and Klumperman, 2009; Settembre et al., 2013). The presence of the v-ATPase, which pumps H+ ions to the lysosomal lumen generates membrane potential. The membrane potential is kept, by the export of cations, and import of anions. In this form, the ATPase activity is maintained (Mindell, 2012). Additionally, several lysosomal ion channels have been described, such as mucolipins, TRP channels (TRPMLs), and two-pore channels (TPCs)(Patel and Docampo, 2010; Xu and Ren, 2015).

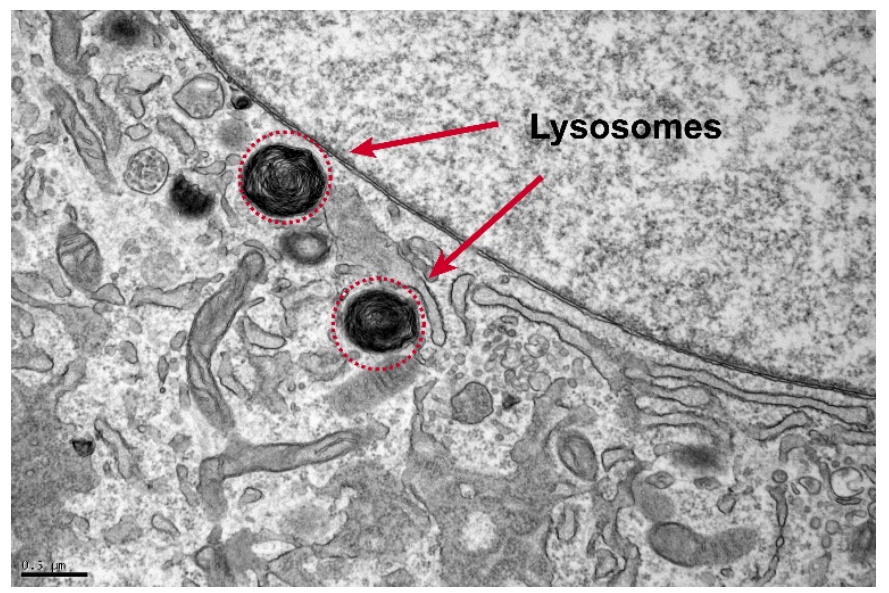

Figure 5. Electron micrograph of mouse embryonic fibroblasts illustrating electron-dense lysosomes. In the image, two lysosomes of different sizes are shown. The presence of multilamellar structures in the lysosomes can be observed. The image was kindly provided by Renata Couto.

\subsubsection{Lysosomal function}

The most recent studies about the lysosome are focused on its role as a central signaling hub that governs cell growth, division, and differentiation (Lawrence and Zoncu, 2019). Plasma membrane receptors, extracellular material, and intracellular material are all processed by the lysosomes. Lysosomes are involved in two of the major processes of degradation for the cell: endocytosis, a significant mechanism of cargo delivery destined for degradation, and autophagy, a process in which the intracellular material is degraded. The final destination of most of the macromolecules is to be digested by the lysosomal hydrolytic enzymes(Kilpatrick et al., 2015).

\subsubsection{Lysosomal degradation of extracellular material}

Once the lysosomal proteins (membrane proteins and enzymes) are synthesized in the endoplasmic reticulum, lysosomal proteins traverse the Golgi complex to enter the trans-Golgi network (TGN), where the majority of lysosomal enzymes are directly sorted to the endolysosomal complex. These enzyme-containing lysosomes will later fuse with endocytic vesicles for the degradation of their cargo. There are several distinct subtypes of endocytic 
vesicles that bud from the plasma membrane and mediate entry to the cell (Kumari et al., 2010). According to their different functions and role in the endocytic pathway, three main endosomes have been reported: early, recycling, and late (Huotari and Helenius, 2011).

Endocytosis frequently starts when clathrin-coated pits formed in the plasma membrane bud into vesicles. Immediately after this process, the vesicles fuse with previously formed early endosomes under the control of the small GTPase Rab5 (Laifenfeld et al., 2007). Early endosomes act as a sorting station, allowing the newly empty receptors to return to the plasma membrane for later use, and directing the now free ligands molecules towards the lysosomes for degradation (System and Klumperman, 2015). When the endocytosis is regulated by Rab4 and Rab11, the receptor together with the membrane-bound lipids send to recycling endosomes and returned to the plasma membrane (Hsu and Prekeris, 2010).

After the early endosomes are formed, internal vesicles start to bud towards the luminal side of these early endosomes. The number of vesicles formed increases, and thus constitute an essential step of maturation for the early endosome. In this process, the multivesicular bodies (MVB) are formed. The maturation process of the MVB is associated with changes in the presence of Rab proteins. Early endosome has Rab5 and late endosomes Rab7 (Poteryaev et al., 2010). The last stage in the endocytosis is the fusion of the late endosomes with the lysosomes. As a consequence of the fusion, the macromolecules inside the endosomes and intraluminal vesicles are degraded.

During the endolysosomal pathway, it has been shown that the luminal $\mathrm{pH}$ decreases, and the amount of hydrolases increases. Although not all enzymes are active against all substrates in the lysosomes and the activity of each enzyme varies (Hu et al., 2015; Pillay et al., 2002).

\subsubsection{Lysosomal degradation of intracellular content}

Autophagy is defined as a conserved catabolic process that is vital for cellular homeostasis. By this process, the cell recycles intracellular macromolecules and organelles and improves the lysosomal degradation. It has been described so far three different types: chaperone-mediated autophagy (unique molecules are selected for degradation into the lysosome by chaperones), microautophagy (cytoplasmic components are directed into the lysosomes), and macroautophagy (cytosolic components are degraded by sequestration into a double-membrane vesicle named autophagosome that later fuses with lysosomes)(Hansen et al., 2018) (Fig. 6).

Microautophagy is a non-selective process for molecules degradation in which the selection of cargo to the lysosomal limiting membrane. During this process, the lysosomal membrane is arbitrarily invaginated and converted into the autophagic tube, which encloses a portion of cytosol. This process is important for the cell; however, the underlying mechanism is still not precise (Li et al., 2012). In the case, the proteins are recognized by the chaperone due to the 
presence of a KFERQ motif ( chaperone-mediated autophagy). In the cytoplasm, the heat shock cognate $71 \mathrm{kDa}$ protein (HSC70) recognizes these motifs and recruits the substrate onto the lysosomal membrane surface. After that, the complex chaperon-protein bind to LAMP-2A and the complex is transferred inside the lysosome (Kaushik and Cuervo, 2018).

The process of macroautophagy is mediated by several autophagy-related (ATG) proteins. It can be divided into at least five consecutive steps: initiation that can be induced by nutrient starvation. After that, the double membrane is formed with a shape of a cup and phagophore is formed. Next, the phagophore is expanded in the cytoplasmic cargo is sequester. In the next step, the phagophore fuse itself forming a structure known as autophagosome which lately fuses to the lysosome. The final step, it is the degradation of sequestered cargo inside of the autolysosome (Hansen et al., 2018).

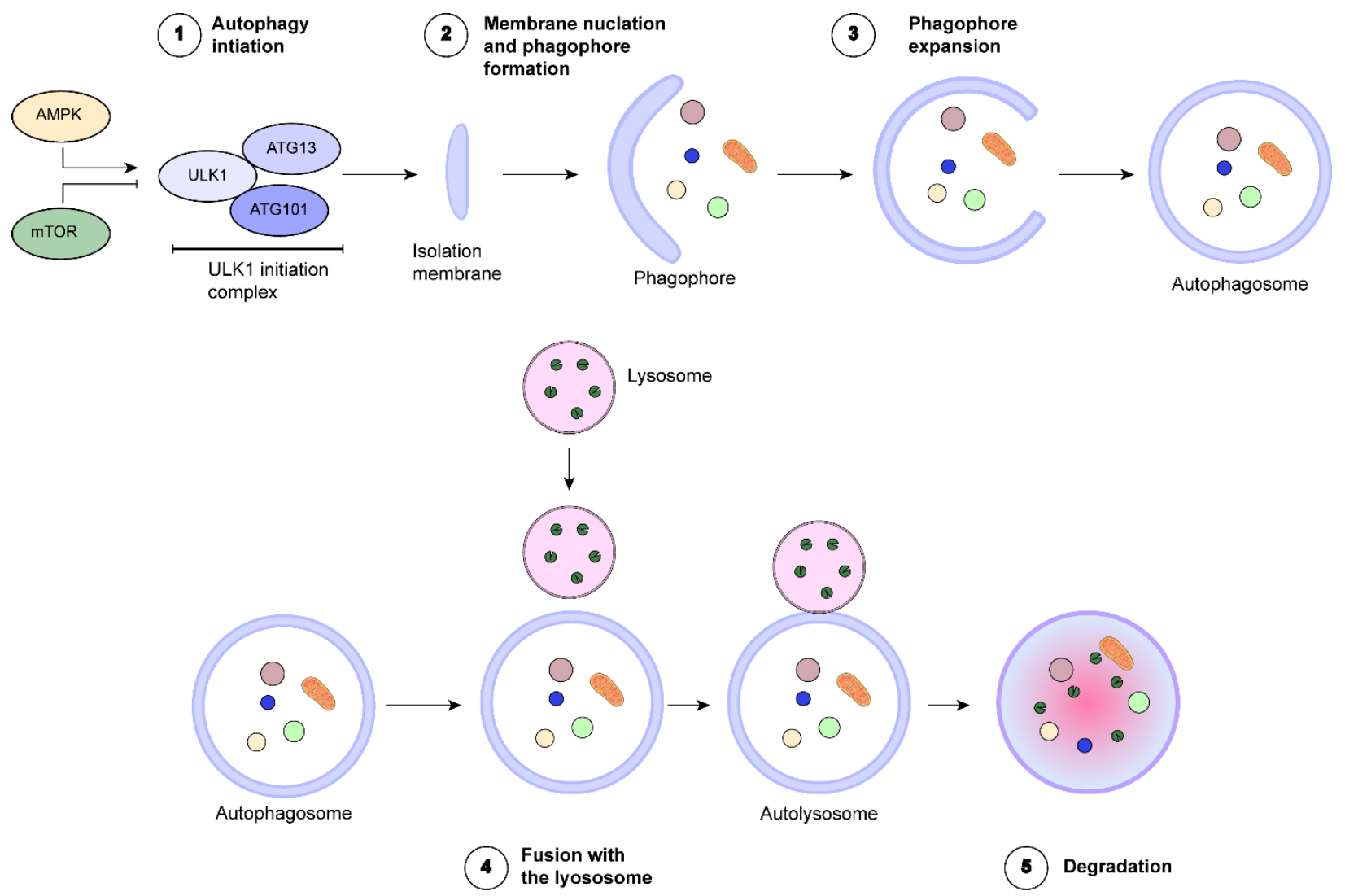

Figure 6. The macroautophagy process. Macroautophagy consists of several steps: (1) initiation induced by nutrient starvation (mTORC inhibits the initiation complex, and AMPK activates it), (2) when the process is active a double membrane structure is formed (membrane nucleation) with a cup-shape form leading to phagophore formation. (3) Phagophore expansion produces the formation of the autophagosome where the macromolecules are sequestered. After the autophagosome formation, the autophagosome fuse with the lysosome (4) generating the degradation (5) of the material inside the autolysosome. The autophagy process is regulated by multiple ATG proteins. Figure adapted from (Hansen et al., 2018). 


\subsubsection{Lysosome in nutrient sensing}

Beyond its role in cellular metabolism through the degradation of macromolecules, the lysosome has emerged as an integration hub for signal transduction, especially in the cellular metabolism and nutrient sensing. It has been discovered that mammalian target of rapamycin (mTOR) protein kinase, one of the master growth regulators, is recruited to the lysosome in response to nutrients in yeast and mammalian cells(Yang et al., 2017).This discovery center the lysosome as in integration hub for the cellular metabolism.

At least two pools of amino acids are needed for the activation of mTOR complex 1 (mTORC1): (i) the cytoplasmic pool and (ii) the pool of amino acids produced from the degradation of macromolecules within lysosome/ vacuole (Perera and Zoncu, 2016). Cytoplasmic amino acids are sensed by SAMTOR, Sestrin, and CASTOR. These proteins modulate the activity of mTORC1 in according to the availability of to their ligands arginine, S-adenosylmethionine, and leucine, respectively (Kim and Guan, 2019). In addition to the cytoplasmic sensing of amino acids pool, mTORC1 senses the lysosomal amino acids availability interacting with its components in the Iysosomal transmembrane. For example, it senses amino acids through v-ATPase and another amino acid transporter, called sodium-coupled neutral amino acid transporter 9 (SLC38A9)(Wyant et al., 2017). Additionally, new evidence suggests that mTORC senses the inputs from glucose and cholesterol. In the case of cholesterol and arginine, mTORC1 is activated by SLC38A9; in this way, a multi-mode nutrient integration is possible (Castellano et al., 2017).

Once mTORC1 is active at the lysosomal membrane, it activates S6-kinase by phosphorylation, promoting the biosynthesis of lipids (Caron et al., 2015), and nucleotides (Robitaille et al., 2013). Also, it promotes a switch metabolism towards the use of glucose and ribosomal biogenesis (Jastrzebski et al., 2007). Simultaneously, the activation of mTORC1 results in the inhibition of two critical initiators of the autophagosome formation (ULK1 and Atg13 protein)(Shen and Mizushima, 2014). Also, on the lysosomal membrane, mTORC1 inhibits the MiT/TFE factors transcription factor EB (TFEB), TFE3, TFEC, and microphthalmia-associated transcription factor (MiTF) by phosphorylation. These transcription factors have been shown to play a crucial role in the autophagic gene expression and lysosomal biogenesis(Martina et al., 2016; Napolitano and Ballabio, 2016).

\subsubsection{Lysosomal storage diseases}

As before mentioned, mutations in genes that encode lysosomal hydrolases can cause several disorders in the body. Theses pathologies constitute a group of diseases named lysosomal storage diseases (LDSs). Also, some of these disorders can be caused by mutations in lysosomal membrane transporters or integral proteins of the membrane. (Futerman and Van Meer, 2004). Nowadays, there are more than 50 recognized LSDs. In most of the LSDs, It is observed in the lysosomes accumulation of substrates none degraded. Because of that, the LSDs are grouped 
according to the chemical nature of the accumulated substrate. It has been reported that approximately 1 in 8000 live births are diagnosticated with one of these disorders, making LSDs one of the most common disorders compared to others rare diseases (Fuller et al., 2006). Because the accumulation of substrate occurs in several organs and systems, the phenotypes observed in the patients are diverse. It depends on the variable visceral association, hematological, ocular, skeletal, and neurological symptoms. Usually, LSDs disorders progress and evolve (Parenti et al., 2013).

The pathologies associated with the LSDs can cause impairments in other lysosomal-related pathways. In Pompe disease, for example, a progressive myopathy triggered by a deficiency in the enzyme acid a-glucosidase (GAA), leads to an expansion of the autophagic compartment. Another disease associated with impairment in autophagy is multiple sulfatase deficiency (MSD). In this case, the fusion between autophagosomes and lysosomes are affected. The primary source of this disease is a defective posttranslational activation of sulfatase-modifying factor 1 (SUMF-1). One of the most studied cases of LSDs induced by a deficiency in the transport of substrates is Niemann-Pick disease (NP). There are three subclasses of Niemann-Pick disease. Initially, all of them were described as sphingomyelin-storage disorders. However, it was discovered that only Niemann-Pick types A and B are produced by the defective activity of sphingomyelinase. Niemann-Pick type C (NPC) is originated by a deficient transport of the cholesterol, that is caused by a deficient activity of NPC1 (cholesterol transporter) or by the inefficient binding of the soluble portion (NPC2 protein) to cholesterol in the lysosomal lumen (Futerman and Van Meer, 2004; Parenti et al., 2015). In this study, two main LSDs are relevant and will be discussed in the succeeding sections.

\subsubsection{Pompe's Disease}

The disease was first discovered in 1932 by Johannes Pompe. Analyzing tissue samples of a child who died from cardiomyopathy, he observed ubiquitous deposition of glycogen in vacuoles (later known as lysosomes). By 1963, H. G. Hers classified this pathology as a glycogen storage disease type II and determined that the deposits were a consequence of the deficient activity of acid alpha-glucosidase (Cabello and Marsden, 2016). The main reason for this disease is mutations in the gene that encodes the acid alpha-glucosidase (GAA) enzyme. More than 300 variations of mutations in the gene have been detected, but the severity of the disease varies according to type of mutation and which region of the gene is affected (Kroos et al., 2008).

One of the main characteristics of the disease is the presence in granules of lysosomal glycogen when cells are observed by electron microscopy. The glycogen accumulation could also be observed in the inter-fibrillar spaces and cytoplasm (Askanas et al., 1976). In some cases, the lysosomes can break, resulting in large "glycogen lakes," which have been associated in the muscle with the pathology of fibrosis and loss of function (Griffin, 1984). Most recently, an 
impairment in the autophagy process was reported in the Gaa $\%$ mouse model, and also in Pompe's disease patients (Raben et al., 2009) proposing that defective autophagy may play a role in the disease. However, autophagy is not the only metabolic process affected by this disease. Reported abnormalities in the calcium homeostasis and inefficient mitochondrial activity were reported in Pompe's disease (Lim et al., 2015).

\subsubsection{Cysteine cathepsins proteases}

In the lysosomes it has been shown the presence of more than 50 different enzymes, all of them are acid hydrolases responsible to the digestion of catabolites. Among these enzymes are the cathepsin (CTS) proteases. There are three main classes of cathepsins: aspartic cathepsins (CTS D and $E$ ), serine cathepsins (CTS A and G), and cysteine cathepsins that comprises 11 members (CTS B, C, F, H, K, L (CTSL1), CTSL2 (CTSV), O, S, W and Z (CTSX)(Cermak et al., 2016; Fonović and Turk, 2014).

Most of the cysteine cathepsins have an endopeptidase activity, cleaving the peptide bonds inside their protein substrates. Two of the cysteine cathepsins have an additional activity, CTSB (carboxypeptidase) and CTSH (aminopeptidase) and another two do not present endopeptidase activity, CTSC and CTSZ (Devanathan et al., 2005; Nägler et al., 1999). The enzymatically active site is formed by a histidine, a cysteine, and an asparagine residue. This three residue constitutes a classic acid-base-nucleophile triad. Additionally, it has been shown that most of these enzymes are glycosylated (Ghosh et al., 2003).

Cysteine cathepsins are proteases conserved since prokaryotes, due that they belong to the superfamily of papain proteases (Olson and Joyce, 2015). Two members of this family, CTSB and CTSL have been linked to several diseases such as cancer, osteoporosis, infectious diseases, and rheumatoid arthritis (Ishibashi et al., 1999; Troen). In the context of cancer, it has been found that cells can secret CTSB and CTL into the extracellular space due to alterations in endolysosomal trafficking (Olson and Joyce, 2015). Once secreted cathepsins modified the tumor microenvironment degrading the extracellular matrix promoting the tumor invasion (Aggarwal and Sloane, 2014). Furthermore, it was reported that mice lacking both of these cathepsins present neurodegeneration and pronounced reactive astrocytosis (Felbor et al., 2002), two main hallmarks of Alzheimer's disease and NPC. Another characteristic of these cathepsins is that their inhibition perturbs cholesterol metabolism and produces a phenotype similar to NPC (Cermak et al., 2016). 


\subsection{Biological membranes}

\subsubsection{Structure of the membrane}

In 1972, Jonathan Singer and Garth Nicolson defined the fluid mosaic model which describes the dynamic and fluid nature of biological membranes. The analogy of the mosaic is represented by the proteins inserted into the fluid, which is the lipid bilayer. The bilayer allows the lateral diffusion of proteins and lipids through the membrane surface. Usually, lipids move faster than the proteins (Zimmerberg and Gawrisch, 2006) (Fig. 7). Conditions such as membrane composition and temperature affect the fluidity of the lipid bilayer.

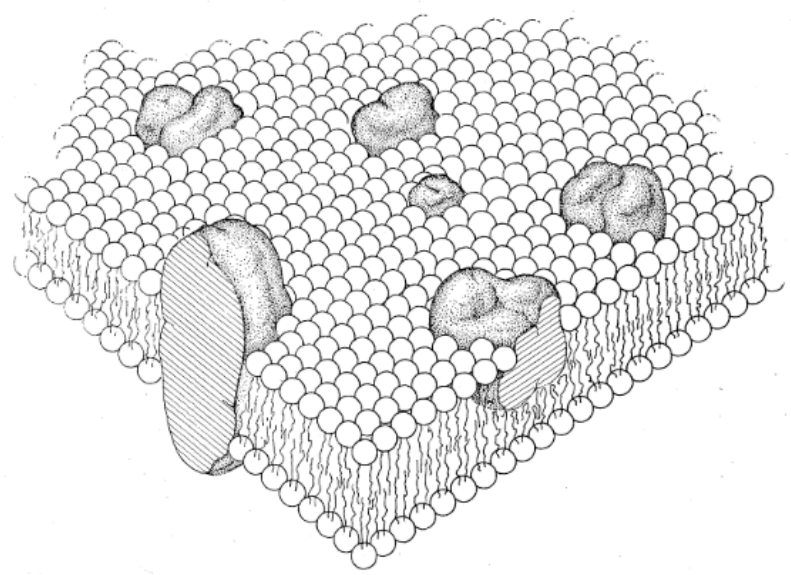

Figure 7. Model of the fluid mosaic. Proteins and lipids are essential components of the membranes. Hydrophobic lipid "heads" are facing the outside part of the membrane; meanwhile, the hydrophilic part is facing the inside forming a matrix where proteins are positioned; schematic three-dimensional and cross-section views (Singer and Nicolson, 1972).

\subsubsection{Lipid composition}

Biological membranes are formed mainly three different types of lipids: sterols, phospholipids, glycolipids. Phospholipids have two hydrophobic tails connected to glycerol and a polar head group containing a phosphate group. When the phospholipid has a three-carbon glycerol backbone, it is called phosphoglyceride. One of the most abundant phospholipids is the phosphatidylcholine (PC) (Fig. 8a), in which a choline molecule is liked to the phosphate group. Reemplazament of the choline with serine produces phosphatidylserine (PS). In case that the choline is replaced by ethanolamine, phosphatidylethanolamine (PE) is formed. The last group of lipids in the glycolipids that contain either glycerol or sphingosine and the phosphate head is replaced by a sugar such as glucose. (Fig. 8b). Sterols are essential for the composition of animal 
membranes, being the most important the cholesterol. The structure of cholesterol is quite different compared to the other two groups. To four-ring steroid structure, a short hydrocarbon side chain and hydroxyl group (which is the hydrophilic 'head') are added (Fig. 8c) (Watson, 2015).

A

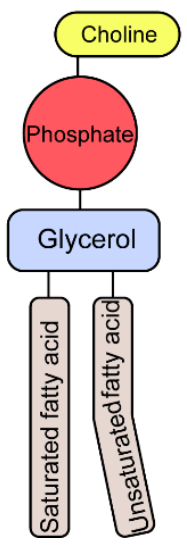

B

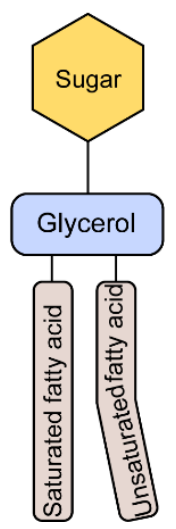

C

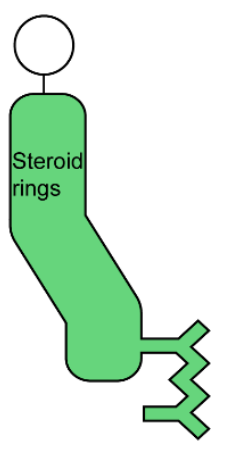

Figure 8. Schematic representation of the three types of membrane lipids. The lipidic composition of the membrane, phospholipid content, and sterol content can be modified by mammalian cells. A) Phosphatidylcholine, B) Glycolipid, and C) a sterol. Figure adapted from (Watson, 2015).

The distribution of lipids in the membrane is not random. Each organelle has a different lipidic composition. This composition affects the structure, shape, and function (Dowhan, 1997). The lipid composition of different organelles can be appreciated in the following table (Table 1):

\begin{tabular}{|c|c|c|c|c|c|}
\hline & Mitochondria & $\begin{array}{c}\text { Endoplasmic } \\
\text { Reticulum }\end{array}$ & Lysosomes & Golgi & $\begin{array}{c}\text { Plasma } \\
\text { membrane }\end{array}$ \\
\hline Phospholipid (mg/mg protein) & 0.175 & 0.374 & 0.156 & 0.825 & 0.672 \\
\hline \multirow[t]{2}{*}{ Sterols (mg/mg protein) } & 0.003 & 0.014 & 0.038 & 0.038 & 0.128 \\
\hline & $\%$ of total phosphc & pids & & & \\
\hline Phosphatidylcholine & 44 & 60 & 48 & 51 & 40 \\
\hline Phosphatidylethanolamine & 34 & 23 & 17 & 21 & 24 \\
\hline Phosphatidylinositol & 5 & 10 & 6 & 12 & 8 \\
\hline Phosphatidylserine & 1 & 2 & 3 & 6 & 9 \\
\hline Cardiolipin & 14 & 1 & 1 & 1 & 1 \\
\hline Phosphatidic acid & $<1$ & 1 & 1 & $<1$ & 1 \\
\hline Sphingomyelin & 1 & 3 & 24 & 8 & 7 \\
\hline
\end{tabular}

Table 1. Lipidic composition of a subcellular fraction of rat liver. Data from Lipids of mitochondria (Voelker, 2005). 
One of the most studied membranes is the mitochondrial membrane. The main characteristics of the mitochondrial membrane are i) the proportion of lipids (phospholipid and sterol) to proteins are lower compared to other subcellular fractions. ii) About $80 \%$ of the total phospholipids present in the membrane are phosphatidylcholine (PC) and phosphatidylethanolamine (PE). iii) The membrane has a high content of cardiolipin (between $10-15 \%)$. iv) The proportion of sphingolipids and sterols is low in this membrane. See Table 1.

Since the membrane sterol content is also low, small changes in the sterol content in the membrane is critical. An increase of cholesterol requirement has been reported in cases of high cell proliferation (Bensinger et al., 2008; Lo Sasso et al., 2010) and tumor growth (Borena et al., 2012; Clendening et al., 2010). In pathological conditions, cholesterol can be accumulated in the mitochondrial membranes modifying the membrane organization. The increase of cholesterol in the membrane alters the permeability and function of resident proteins (Maxfield and Tabas, 2005). For example, increment in cholesterol content of the mitochondrial membrane has been observed in cases of cancer (Ribas et al., 2016) and lysosomal storage diseases (LSD), especially in Niemann Pick C (NPC type C) (Balboa et al., 2017).

\subsubsection{Cholesterol}

\subsubsection{Structural importance of cholesterol in membranes}

The molecule of cholesterol (Fig. 9) is composed of a four-ring skeleton fused to a hydroxyl group at carbon 3 (Urich, 1994). The hydroxyl group is of great importance because it gives to cholesterol the amphiphilic character allowing the cholesterol molecule to orient in membranes (Bloch, 1983). The permeability properties of the lipid bilayer are enhanced when cholesterol is mixed with phospholipids (Cooper and Shattil, 1980). Cholesterol inserts into the bilayer with its hydroxyl group close to the polar head groups of the phospholipids (Ohvo-Rekilä et al., 2002). In this position, cholesterol decreases the mobility of the hydrophobic tails of the phospholipids (Yeagle, 1985). As a result, there is a reduction in the permeability of the membrane to small molecules water-soluble since the lipid bilayer became more rigid in this region (Needham and Nunn, 1990). 


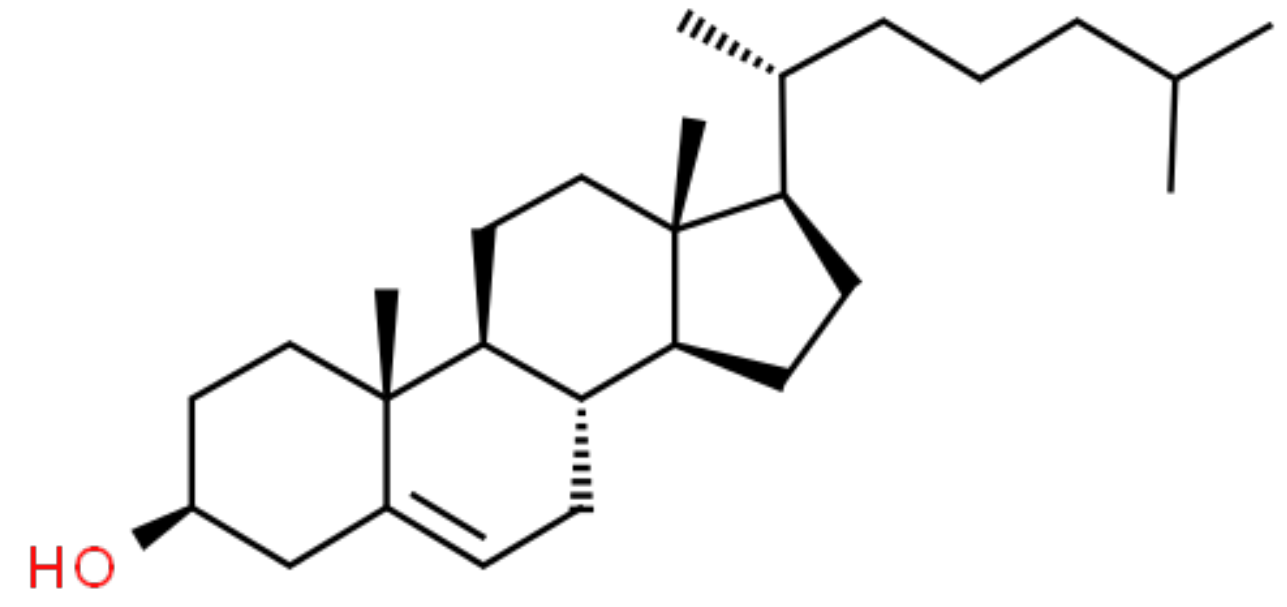

Figure 9. 2D structure of a cholesterol molecule. The essential hydroxyl group, which gives to cholesterol the orientation in biological membranes is in red. The structure was obtained from ChemSpider

\subsubsection{Cholesterol biosynthesis pathway}

There are two main ways to obtain cholesterol: (i) through the ingestion of particular food or (ii) de novo synthesis (Brown et al., 2018).

i) Cholesterol is obtained by the ingestion of food of animal origin. Cholesterol and cholesteryl esters are delivered to many tissues by low-density lipoprotein (LDL) (Goldstein and Brown, 2015). High- density lipoprotein (HDL) is responsible for the removal of free cholesterol and its transport to the liver (Brown and Goldstein, 1986).

ii) The cholesterol biosynthesis could be studied as a process that consists of five major steps (Boyle, 2005): 1) acetyl-CoA is converted to mevalonate 2) mevalonate is used as a precursor of isoprenoid units 3) six isoprenoid units are condensed into squalene 4) the primary steroids structure if formed by squalene cyclization forming lanosterol 5) lanosterol is used to produce cholesterol (Fig. 10) (Cerqueira et al., 2016).

Step 1) Synthesis of mevalonate from acetate: two molecules of acetyl-CoA are condensed to produce acetoacetyl-CoA catalyzed by cytosolic thiolase. The produced acetoacetyl-CoA is condensed with another molecule of acetyl-CoA in a new reaction. This process is catalyzed by HMG-CoA synthase. This new product is named HMG-CoA. In the last step of this stage, HMGCoA to mevalonate by HMG-CoA reductase (Fig. 10). In the last step, two molecules of NADPH donate two electrons. The conversion of mevalonate to HMG-CoA is the primary regulatory step in the cholesterol biosynthesis pathway (Clendening et al., 2010). Because of that, the inhibition of HMG-CoA reductase with drugs is one of the most effective treatments to reduce cholesterol levels (Sharpe and Brown, 2013). 
Step 2) Conversion of mevalonate to two isoprenes: mevalonate is phosphorylated sequentially by three different kinases: mevalonate kinase, phosphomevalonate kinase, and mevalonate pyrophosphate decarboxylase. Initially, the mevalonate is phosphorylated by the three different kinases to form an intermediate named 3-Phospho-5-pyrophosphomevalonate. From this intermediate, when the carboxyl and the phosphate group leave a new structure of double bond is produced. In this way, $\Delta 3$-isopentenyl pyrophosphate is formed, and the isomerization of the $\Delta 3$-isopentenyl pyrophosphate generates the other isoprene, dimethylallyl pyrophosphate (Fig. 10).

Step 3) Six molecules of activated isoprene are condensed forming squalene: dimethylallyl pyrophosphate and isopentenyl pyrophosphate are condensed, removing one phosphate group and forming a 10-carbon chain compound, geranyl pyrophosphate. This new molecule undergoes another condensation reaction with isopentenyl pyrophosphate, yielding a 15-carbon molecule, farnesyl pyrophosphate. In the last stage, two molecules of farnesyl pyrophosphate join to create squalene (Fig. 10) while removing both pyrophosphate groups.

Step 4) Four-ring steroid nucleus is formed by squalene cyclization: the folding of squalene form a structure closely similar to the steroid nucleus. To be able to form the ring, squalene must be converted to squalene 2,3-epoxide by an oxidase, squalene epoxidase. Squalene 2,3-epoxide is cycled by lanosterol synthase resulting in lanosterol as the final product (Fig. 10).

Step 5) Formation of cholesterol: the conversion of lanosterol to cholesterol consists on a series 19-steps process catalyzed by nine different enzymes: two enzymes are employed in multiple steps, and three are used two times in the process. To obtain cholesterol, several methyl groups migrate, and others are removed from lanosterol (Risley, 2009). 


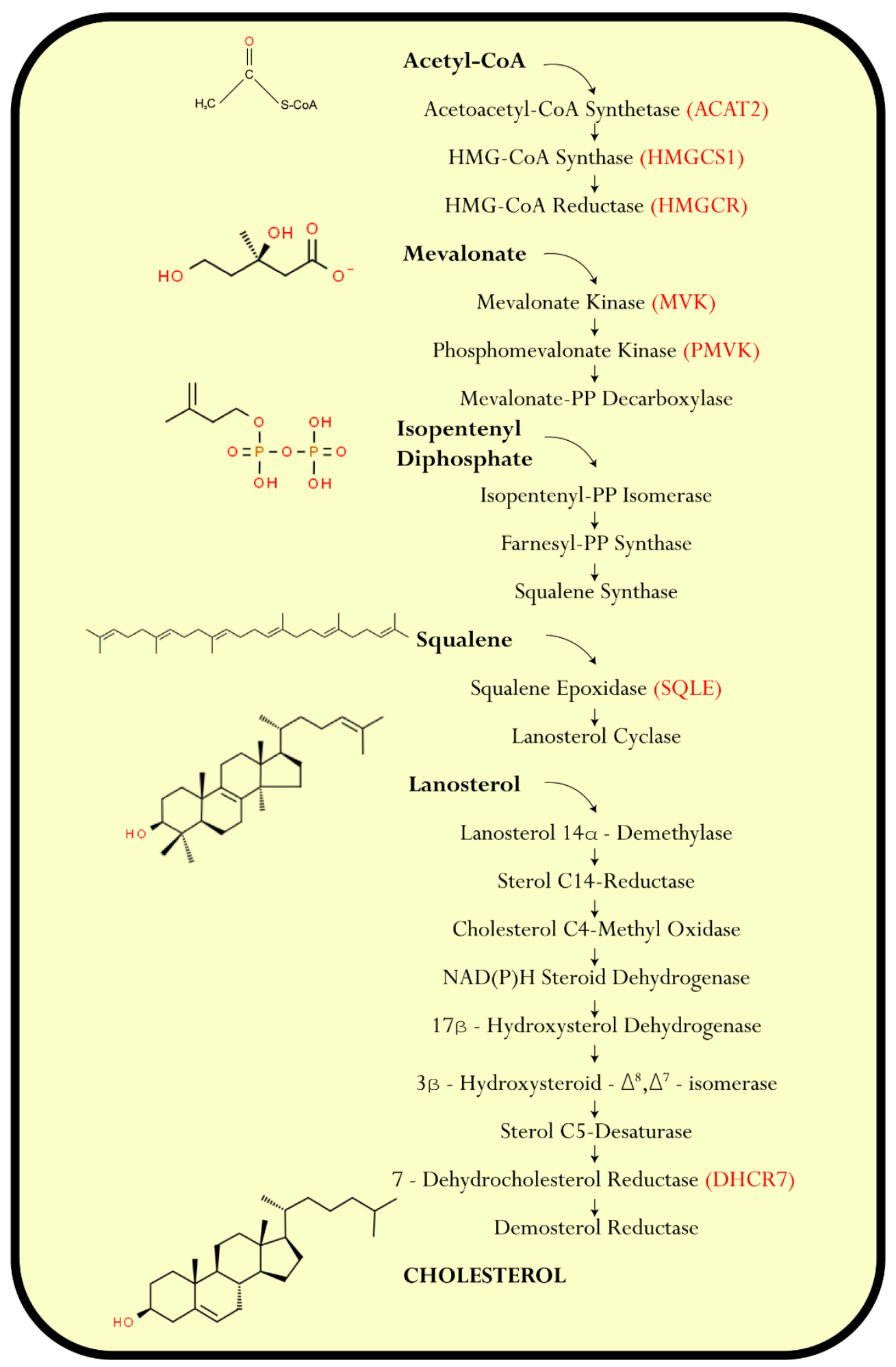

Figure 10. The mammalian cholesterol biosynthetic pathway. The synthesis of cholesterol from acetyl-CoA requires at least 20 enzymes. SREBPs regulate the expression of the genes encoding these enzymes. The four central intermediates (mevalonate, isopentenyl diphosphate, squalene, and lanosterol) are in bold. The primary genes studied during this thesis are in red brackets. Lipids structures were obtained from ChemSpider. 


\subsubsection{Regulation of cholesterol biosynthesis}

\subsubsection{Sterol regulatory-element binding proteins (SREBPs).}

Sterol regulatory-element binding proteins (SREBPS) are a conserved family of transcription factors that regulates the expression of genes necessaries for the lipid homeostasis according to requirements of each species. In mammals, there are three different isoforms of SREBP: SREBP1a, SREBP-1c, and SREBP2 (Brown and Goldstein, 1997). By alternative splicing of the same gene, SREBP-1a and SREBP-1c are generated (Horton et al., 2002).

The network of genes regulated by SREBPS, regulate the activity of the transcription factor at multiple levels by feedback regulation (Hitoshi Shimano and Sato 2017). Each different SREBP protein has a determined physiological role. For example, lipid synthesis in rapidly growing cells is fully activated by SREBP1a, whereas SREBP1c is involved in fatty acid synthesis and energy storage (H Shimano et al. 1997; Toth et al. 2004). SREBP2 mostly regulates the transcription of genes related to cholesterol metabolism and mediates sterol regulation in every tissue (Horton, Goldstein, and Brown 2002).

SREBPs are located in the membrane of the endoplasmic reticulum (ER), and the active form is generated once they are cleaved in the Golgi. Once active, they are transported to the nucleus by importin $\beta$. Although the normal process requires binding of the proteins to importins $\alpha$ and $\beta$, SREBPs can bind importin $\beta$ even in without importin $\alpha$. However, before entering the nucleus, the SREBP helix-loop-helix leucine-zipper domain needs to be dimerized (Nagoshi et al. 1999). When the complex is transported to the nucleus, it is fast degraded by the ubiquitin and proteasome pathway (UPS). It has been reported that cultured cells treated with proteasomes inhibitors showed an increase in the nuclear levels of SREBPs and its regulated genes (Hirano et al. 2001). In the nucleus, SREBPs associates with several transcriptional co-activators (e.g., CBP and p300). The interaction of these co-activators with SREBPs increase its transcriptional activity probably because the co-activators have an intrinsic histone acetyltransferase activity. The acetylation of SREBPs inhibits the degradation by the UPS (Giandomenico et al. 2003; Sundqvist and Ericsson 2003).

\subsubsection{Mechanism of activation of SREPBs}

SREBPs protein has two domains, $\mathrm{NH}$-terminal (transcription factor domain) and $\mathrm{COOH}$-terminal (regulatory domain). Both domains are located in the cytosol and connected by a membranespanning helix that flanks a short loop facing the ER lumen (Fig. 11). Immediately SREBP is synthesized in the ER membrane, the protein binds another ER membrane protein, SCAP (SREBP cleavage activating protein), through its $\mathrm{COOH}$ terminal domain in the cytosol (Hua et al., 1996).

The location of SCAP in the ER membrane allows it to sense cholesterol in the membrane. SCAP has an N-terminal domain formed by eight transmembranes (TM) helices separated by 
hydrophilic loops. The TM helices 2-6, are known as a sterol-sensing domain (SSD) (Nohturfft et al., 1998), bind a scaffold protein, Insigs (insulin-induced gene), in the ER membrane, in high cholesterol conditions (Yang et al., 2002). When the cholesterol proportion is more than $5 \%$ of total lipids of the ER membrane, it binds to the luminal loop 1 of SCAP. The binding promotes changes in the conformation of SCAP hiding the luminal loop 6, which is recognized by COPII proteins for transport to Golgi (Goldstein and Brown, 2015). Under this new conformation, the SCAP/SREBP complex is retained in the ER membrane preventing Golgi processing of SREBP (Fig. 4).

Conversely, when the cholesterol content drops to less than 5\%, SCAP/SREBP complex exits the ER. In this condition, loop 1 of SCAP binds loop 7, thus exposing loop 6 (Brown et al., 2018). Once loop six is exposed, it binds COPII-coated vesicles that bud from ER membranes. The vesicles are transported to the Golgi (Fig. 5)(Radhakrishnan et al., 2008). SREBP in the Golgi membrane is cleaved in two different sites yielding the $\mathrm{NH2}$-terminal domain (transcription factor domain) (Brown and Goldstein, 1997).
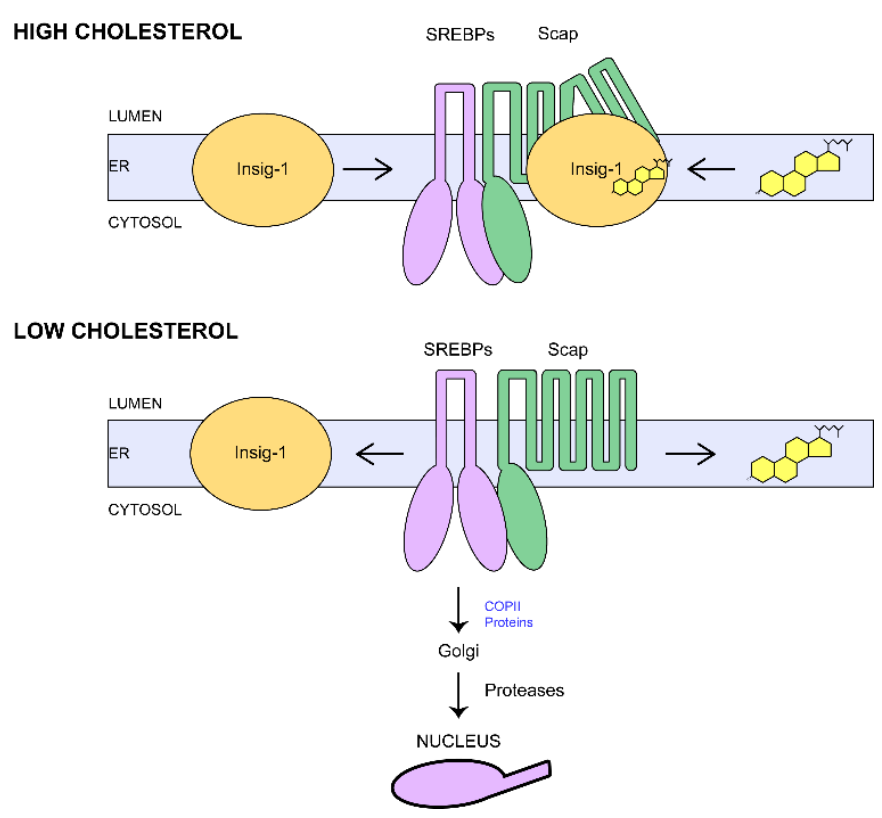

Figure 11. Model of regulation of SREBP in ER membranes. Under conditions of high cholesterol in the cell, Insig-1 binds to SCAP/SREBP complex retaining the complex in the ER membrane. However, in cases of low cholesterol, Insig-1 does not bind the complex SCAP/SREBP. Because of that, SCAP/SREBP are transferred to the Golgi, where SREBP is processed. Illustration based on (Goldstein et al., 2006).

\subsubsection{Control of lipids biosynthesis}


Several transcription factors activated by extracellular stimuli have been reported to modulate the activity of SREBPs. For example, hepatocyte nuclear factor 4 (HNF4) and the liver X receptor (LXR) upon activation both nuclear receptors bind SREBPs regulating its activity. These nuclear receptors are activated by the binding of lipophilic ligands. The regulation of the lipid biosynthesis pathway by HNF4 and LXR generates an additional mechanism for the maintenance of lipid homeostasis maintenance (Kanayama et al., 2007).

Lipogenesis is an anabolic process by which fatty acids are synthesized from acetyl-CoA. Insulin was discovered as a significant anabolic hormone that controls lipogenesis (Shimomura et al., 1999). Initially, the activation of SREBP1 by insulin was identified to be regulated by PI3K and AKT, and these two kinases are induced by insulin (Krycer et al., 2010). Later, mammalian target of rapamycin (mTOR) emerged as a critical anabolic integrator that controls multiple cellular processes such as autophagy, cell growth, cell cycle, and cell survival (Zoncu et al., 2011). mTOR is a serine/threonine-protein kinase conserved through the evolution that is present in the cell as two different complexes mTORC1 and mTORC2 (Loewith et al., 2002). Both of them are formed from several proteins. mTORC1 contains mTOR, Raptor, mLST8, and PRAS40; meanwhile, mTORC2 is formed by mTOR, Rictor, mSin1, Protor, and mLST8 (Caron et al., 2015). The activation of mTORC1 is controlled by nutrient status and cellular energy. Meanwhile, mTORC2 is dependent on extracellular signals, mainly growth factors and cytokines, for the final activation of mTORC1 (Ebner et al., 2017). mTORC1 is an essential downstream hub of integration for the signaling pathways induced by insulin. It controls the three major nutritional pathways: protein,glucose, and lipid metabolism (Shimano and Sato, 2017).

There are several molecular mechanisms involved in the activation of SREBPs signaling induced by insulin, for example, through AKT activation. Once active, AKT inhibits by phosphorylation the hepatic insulin-suppressive (INSIG) isoform INSIG2A reducing SREBP stability (Yabe et al., 2003; Yecies et al., 2011). However, there are other mechanisms in which AKT is not involved. One of them is through the activation of mTORC1 by the inhibition of TSC, being TSC an upstream inhibitor of mTORC1 (Porstmann et al., 2008). Another mechanism is that mTORC1 activation leads to phosphorylation and translocation from the nucleus of acid phosphatase lipin-1 (lipin-1 is an inhibitor of SREBP1) (Peterson et al., 2011).

Another essential kinase for metabolism regulation is AMP-activated protein kinase (AMPK). The first metabolic pathway found to be regulated by AMPK was the lipids and sterol synthesis (Herzig and Shaw, 2018). AMPK is a conserved sensor of the energy supply and demand for the cell, and it modulates the growth of organs and cells (Hardie, 2007). This protein belongs to the family of AMPK-related kinase, which is constituted by 13 kinases in the human genome and all of them are serine/threonine-protein kinase. The AMPK is integrated by three different subunits, an $\alpha$ subunit, a $\beta$ subunit, and a $\gamma$ subunit. The catalytic subunit is $\alpha$ while $\beta$ forms the scaffolding unit. A regulatory subunit involved in nucleotide binding is formed by the $\gamma$ subunit (Sanz et al., 2013). 
In mammals, there are multiple subtypes of each subunit, and they are differentially expressed according to the tissues. The catalytic subunit $\alpha$ has two different isoforms ( $\alpha 1$ and $\alpha 2$ ), $\beta$ have two ( $\beta 1$ and $\beta 2)$, and $\gamma$ subunits three $(\gamma 1, \gamma 2$, and $\gamma 3)$ isoforms (Herzig and Shaw, 2018).

In hepatocytes, AMPK can directly phosphorylate SREBP1c or SREBP2 (Li et al., 2011). AMPK phosphorylates SREBP1c at Ser372, inhibiting the cleavage and preventing nuclear translocation, and transcriptional activity; and ultimately suppress lipogenesis (Han et al., 2019; Li et al., 2011). AMPK regulates HMGCR (a vital enzyme of the de novo cholesterol biosynthesis pathway) by phosphorylation at Ser872, close to the C-terminus (Hardie et al., 1998). Its phosphorylation by AMPK represses cholesterol synthesis, a high energy consumption process (Mohamed et al., 2015). It is worth pointing out that HMGCR is present in the cells in both forms, unphosphorylated (active) and phosphorylated (inactive) (Sharpe and Brown, 2013). 


\subsection{Aims}

Models of lysosomal and mitochondrial dysfunction have been intensely studied and the mechanisms underlying the crosstalk between these organelles, still rather elusive, have been the subject of interest of our research group (Fernandez-Mosquera et al., 2019; Yambire et al., 2019). However, less is known about how the dysfunctions of these organelles modify the biosynthesis pathway of some biomolecules such as lipids. Lipids are essential components of cellular membranes and signaling (Jaishy and Abel, 2016). Therefore, this study focuses on the metabolism of cholesterol, especially in its biosynthesis pathway; exploring the independent roles of lysosomal and mitochondrial dysfunction in the biosynthesis of cholesterol. For this reason, we proposed the following aims:

- Establish if the activity of cholesterol biosynthesis is sensitive to mitochondrial and lysosomal dysfunction.

- Characterize transcript level changes of the main enzymes involved in cholesterol biosynthesis pathway in several models of mitochondrial and lysosomal dysfunction.

- Determine changes in the total cholesterol content and unesterified cholesterol content, in the whole-cell and mitochondria, due to mitochondrial and lysosomal dysfunction.

- Identify the main signaling pathways affected by mitochondrial or lysosomal dysfunction and how they affect the cholesterol biosynthesis pathway. 


\section{MATERIALS AND METHODS}

\subsection{Materials}

\subsubsection{Consumables and Kits}

The consumables and kits used during this project are listed below, supplier and catalog number are included.

Table 2. List of consumables and kits

\begin{tabular}{|c|c|c|}
\hline Product & Supplier & $\begin{array}{l}\text { Catalog } \\
\text { number }\end{array}$ \\
\hline Amersham Hybond PO45 PVDF & GE Healthcare & 10600023 \\
\hline Amplex ${ }^{\mathrm{TM}}$ Red Cholesterol Assay Kit & Thermo Scientific & A12216 \\
\hline Centrifuge tubes $15 \mathrm{~mL}$ Corning CentriStar & Corning & 430791 \\
\hline Centrifuge tubes $50 \mathrm{~mL}$ Corning CentriStar & Corning & 430829 \\
\hline Chloroform & Merck & 102442 \\
\hline Cryomold (tissue tek) & Fisher Scientific & NC9511236 \\
\hline CRYSTAL RNA Mini Kit & New England Biolabs & $31-010-404$ \\
\hline Cuvettes PMMA & VWR-International & $634-0678$ \\
\hline DMEM high glucose + pyruvate & Gibco & $41966-029$ \\
\hline DMEN high glucose & Gibco & $41965-062$ \\
\hline Fetal Bovine Serum Heat-Inactivated & Gibco & $10500-064$ \\
\hline $\begin{array}{l}\text { High-Performance chemiluminescence } \\
\text { film }\end{array}$ & GE Healthcare & 28906837 \\
\hline iScrip cDNA Synthesis Kit & Bio-Rad & $170-8891$ \\
\hline Lenti $X$ concentrator & Clontech & 631231 \\
\hline Lipofectamine 2000 Reagent & Invitrogen & $11668-019$ \\
\hline $\begin{array}{l}\text { Luminata }{ }^{\mathrm{TM}} \text { Classico Western HRP } \\
\text { Substrate }\end{array}$ & Millipore & WBLUC0500 \\
\hline $\begin{array}{l}\text { Luminata }{ }^{\mathrm{TM}} \text { Crescendo Western HRP } \\
\text { Substrate }\end{array}$ & Millipore & WBLUC0500 \\
\hline Luminata $^{\mathrm{TM}}$ Forte Western HRP Substrate & Millipore & WBLUF0500 \\
\hline
\end{tabular}




\begin{tabular}{|c|c|c|}
\hline Luna $^{\circledR}$ Universal qPCR Master Mix & New England BioLabs & M3003X \\
\hline Medical X-Ray Film & Foma & \\
\hline Microseal B seal & Bio-Rad & MSB1001 \\
\hline PageRuler Plus Prestained Protein Ladder & Thermo Scientific & 26619 \\
\hline PBS tablets & Sigma Aldrich & P44177-100TAB \\
\hline $\begin{array}{l}\text { PCR plate 384-well skirted ABI-Type } \\
\text { (Universal) }\end{array}$ & StarLab & E1042-3840 \\
\hline Penicillin/Streptomycin & Gibco & $15140-062$ \\
\hline Pierce BCA Protein Assay Kit & Thermo Scientific & 23225 \\
\hline Protein Assay Dye Reagent Concentrate & Bio-Rad & $500-0006$ \\
\hline Serological Pipette $10 \mathrm{~mL}$ & StarLab & E4860-1011 \\
\hline Serological Pipette 25 mL & StarLab & $E 4860-2511$ \\
\hline Serological Pipette $5 \mathrm{~mL}$ & StarLab & E4860-0511 \\
\hline Test Tube Soda Glass & VWR-International & $212-003$ \\
\hline Tips 10 pL TipOne & StarLab & S111-3210 \\
\hline Tips 1000 pL TipOne & StarLab & S1111-6001 \\
\hline Tips 20 pL TipOne & StarLab & S120-1810 \\
\hline Tips 200 pL TipOne & StarLab & S1120-8800 \\
\hline Triple Express (1X) & Gibco & $12605-010$ \\
\hline Trypan Blue 0.4\% & Life Technologies & T10282 \\
\hline Tubes $0.5 \mathrm{~mL}$ molecular probes & Life Technologies & Q33856 \\
\hline Tubes $1.5 \mathrm{~mL}$ & StarLab & E1415-1500 \\
\hline Tubes $2 \mathrm{~mL}$ & StarLab & S1620-2700 \\
\hline Tubes 8 Twin Strip Start PCR & StarLab & $11402-3700$ \\
\hline Western Blot Paper & TH-Geyer & $4-01-60-0041$ \\
\hline
\end{tabular}




\subsubsection{Regents}

List of reagents use during this study

Table 3. List of reagents

\begin{tabular}{|c|c|c|}
\hline Product & Supplier & Catalog Number \\
\hline 2-Mercaptoethanol & Carl Roth & 4227.3 \\
\hline Absolute ethanol BD & Bioscience & 354052 \\
\hline Acrylamide solution $40 \%$ & AppliChem & A0385 \\
\hline Ammonium Persulfate (APS) & AMRESCO & 0486 \\
\hline Antimycin & Sigma Aldrich & A8674 \\
\hline Bovine serum albumin (BSA) & Sigma Aldrich & A7906 \\
\hline Bromophenol blue & Carl Roth & T116.1 \\
\hline $\mathrm{CaCl}_{2}$ & Alfa Aesar & L13191 \\
\hline Chloroquine disphosphate & Sigma Aldrich & C6628 \\
\hline Coomassie Brilliant Blue $\mathrm{G}$ & Carl Roth & 9598.2 \\
\hline Cryo-OCT compound & Fisher Scientific & $14-373-65$ \\
\hline DMSO & Sigma Aldrich & D8418 \\
\hline DTT & AppliChem & A2948 \\
\hline EDTA & Carl Roth & 8043.2 \\
\hline EGTA & Sigma Aldrich & E3889 \\
\hline Ethanol $70 \%$ & VWR & 84858.440 \\
\hline Filipin & Sigma Aldrich & F4767 \\
\hline Glycerol & Carl Roth & 7530.1 \\
\hline Glycine & VWR & 0167 \\
\hline $\mathrm{H}_{2} \mathrm{O}$ Nuclease Free & VWR & E476 \\
\hline $\begin{array}{l}\text { HaltTM Protease \& } \\
\text { Phosphatase single-use } \\
\text { Inhibitor Cocktail }\end{array}$ & Thermo Scientific & 78442 \\
\hline $\mathrm{HCl}$ & Sigma Aldrich & $\mathrm{H} 1758$ \\
\hline
\end{tabular}




\begin{tabular}{|c|c|c|}
\hline HEPES & Carl Roth & HN77.2 \\
\hline $\begin{array}{l}\text { HPCD (2-Hydroxypropyl)- } \beta \text { - } \\
\text { cyclodextrin }\end{array}$ & Sigma Aldrich & H107 \\
\hline $\mathrm{K}_{2} \mathrm{HPO}_{4}$ & Carl Roth & P749.2 \\
\hline $\mathrm{KCl}$ & Volu-Sol & 83608.26 \\
\hline $\mathrm{KH}_{2} \mathrm{PO}_{4}$ & Carl Roth & 3904.1 \\
\hline Methanol & VWR & 20903.368 \\
\hline $\mathrm{MgCl}_{2}$ & VWR & 8.14733 .0100 \\
\hline $\mathrm{MgSO}_{4}-7 \mathrm{H}_{2} \mathrm{O}$ & Sigma Aldrich & 63138 \\
\hline $\mathrm{NaCl}$ & AppliChem & $\mathrm{A} 1430,0010$ \\
\hline $\mathrm{NaH}_{2} \mathrm{PO}_{4} \cdot \mathrm{H}_{2} \mathrm{O}$ & Carl Roth & K300.1 \\
\hline $\mathrm{NaHCOs}$ & Carl Roth & P029.3 \\
\hline $\mathrm{NaN}_{3}$ & Sigma Aldrich & $52002-1006$ \\
\hline $\mathrm{N}$-dodecylmaltoside & Carl Roth & CN26.2 \\
\hline $\mathrm{NH}_{4} \mathrm{Cl}$ & Carl Roth & K298.1 \\
\hline Nonidet ${ }^{\circledR} \mathrm{P}-40$ Substitute & AMRESCO & E109-50ML \\
\hline PMSF & Sigma & P7626 \\
\hline Rotenone & Sigma & R8875 \\
\hline SDS & Sigma Aldrich & L4509-500G \\
\hline Skim Milk Powder & Fluka & 70166 \\
\hline Sodium deoxycholate & Sigma Aldrich & 30970 \\
\hline TEMED & Sigma Aldrich & T7024 \\
\hline Torin-1 & BioVision & 2273 \\
\hline TRI Reagent & Sigma & T9424 \\
\hline Tris Base & Sigma Aldrich & T1503 \\
\hline Tris- $\mathrm{HCl}$ & Carl Roth & 9090.2 \\
\hline Triton X-100 & AMRESCO & 0694 \\
\hline Tween 20 & AMRESCO & 0777-1L \\
\hline
\end{tabular}




\subsubsection{Solution and buffers}

Solutions and buffers used during this study are listed below. All of them were prepared in $\mathrm{dd}_{2} \mathrm{O}$ unless a different solvent is specified.

Table 4. List of solution and buffers

\begin{tabular}{|c|c|}
\hline Buffer/solution & Composition \\
\hline Blocking buffer & $5 \%(w / v)$ Skimmed milk or BSA in TBST \\
\hline Freezing medium & $\begin{array}{l}10 \%(v / v) \text { DMSO, } 20 \%(v / v) \text { FBS in DMEM high } \\
\text { glucose }\end{array}$ \\
\hline Mitochondria isolation buffer & $\begin{array}{l}250 \mathrm{mM} \text { Sucrose, } 20 \text { mM Hepes, } 10 \text { mM KCl, } 1.5 \\
\mathrm{mM} \mathrm{MgCl}_{2}, 0.1 \text { mM EDTA, } 1 \text { mM EGTA, pH } 7.5\end{array}$ \\
\hline NaN solution & $10 \% \mathrm{NaN}_{3}$ \\
\hline PBS & Dissolve 5 PBS tablets in $1 \mathrm{~L} \mathrm{dd}_{2} \mathrm{O}$. Autoclave \\
\hline Resolving gel $12 \%$ & $\begin{array}{l}0.375 \mathrm{M} \text { Tris, } \mathrm{pH} 8.8,0.1 \% \text { SDS, } 12 \% \\
\text { Polyacrylamide, } 0.1 \% \text { APS, } 0.04 \% \text { TEMED }\end{array}$ \\
\hline RIPA buffer & $\begin{array}{l}50 \mathrm{mM} \text { Tris pH } 8.0,150 \mathrm{mM} \mathrm{NaCl}, 1 \% \text { Triton X- } \\
100,0.1 \% \text { SDS and } 0.5 \% \text { Sodium deoxycholate } \\
\text { supplemented with fresh } 1 \mathrm{X} \\
\text { protease/phosphatase inhibitor }\end{array}$ \\
\hline Running buffer $5 X$ & $125 \mathrm{mM}$ Tris Base, .96 M Glycine, .5\% SDS \\
\hline SDS loading buffer & $\begin{array}{l}62.5 \mathrm{mM} \text { Tris, pH 6.8, } 50 \% \text { (v/v) Glycerol, } 12 \% \\
\text { (w/v) SDS, } 0.06 \% \text { (w/v) Bromophenol blue. Add } \\
5 \% \text { 2- Mercaptoetanol freshly prior to use }\end{array}$ \\
\hline Stacking gel $4 \%$ & $\begin{array}{l}62.5 \mathrm{mM} \text { Tris pH } 6.8,0.1 \% \text { SDS, } 5 \% \\
\text { Polyacrylamide, } 0.1 \% \text { APS, } 0.1 \% \text { TEMED }\end{array}$ \\
\hline TBST 10X & 200 mM Tris, 1.5M NaCl, 1\% Tween 20, pH 7.4 \\
\hline Transfer buffer $10 \mathrm{X}$ & $\begin{array}{l}250 \text { mM Tris, } 1.92 \text { M Glycine. 1X dilution includes } \\
20 \%(v / v) \text { Methanol }\end{array}$ \\
\hline Whole-cell extraction buffer & $\begin{array}{l}1.5 \% \mathrm{~N} \text {-dodecylmaltoside in PBS supplemented } \\
\text { with fresh } 1 \mathrm{X} \text { protease/phosphatase inhibitor }\end{array}$ \\
\hline
\end{tabular}




\subsubsection{Cell culture materials}

The following cell culture materials were used during this thesis

Table 5. Cell culture materials

\begin{tabular}{|c|c|c|}
\hline Product & Supplier & Catalog number \\
\hline $60 \times 20 \mathrm{~mm}$ TC dish & CytoOne & CC7682-3354 \\
\hline $100 \times 20 \mathrm{~mm}$ TC dish & CytoOne & CC7682-3394 \\
\hline $150 \times 20 \mathrm{~mm}$ TC dish & CytoOne & CC7682-3617 \\
\hline 6-well TC plate & CytoOne & CC7682-7506 \\
\hline 96-well TC plate & CytoOne & CC7682-7596 \\
\hline Cell scraper & StarLab & CC7600-0202 \\
\hline $\begin{array}{l}\text { Countess cell counting } \\
\text { chamber slides }\end{array}$ & Invitrogen & C10283 \\
\hline Cryogenic vial $2 \mathrm{~mL}$ & Fisher Brand & 1050026 \\
\hline $\begin{array}{l}\text { Filter syringe } 0.220 \text { Rotilabo } \\
\mathrm{CME}\end{array}$ & Carl Roth & SE2M35I07 \\
\hline Syringe Inject & $\begin{array}{l}\text { Becton. Dickinson and } \\
\text { company }\end{array}$ & 4606205 \\
\hline
\end{tabular}

\subsubsection{Cell lines}

HeLa cells used for the experiments in this study were obtained from ATCC. HEK293T cells, obtained from Prof. Katschinki's Lab, were used as packaging cells for the generation of stable knock downs of CTSB, UQCRC1, and GAA. Primary mouse embryonic fibroblasts were prepared from Gaa KO and Ndufs4 KO mice, respectively, following the procedure previously described (Murdoch et al., 2016). Lamp2 KO MEFs were obtained from Prof. Saftig's Lab. CTSB and CTSL MEFs were a kind gift from Prof. Reinheckel's Lab, and AMPK MEFs were obtained from the Viollet's Lab.

In the case of the primary mouse embryonic fibroblasts, the cells were prepared from Gaa KO and Ndufs 4 KO mice ( and their corresponding wild-type (WT) littermates), respectively, following the procedure previously described (Murdoch et al., 2016) 


\subsubsection{Plasmid}

Transfections were done with the following shRNA plasmids

Table 6. Plasmids used in this thesis

\begin{tabular}{lll}
\multicolumn{1}{c}{ Plasmid } & \multicolumn{1}{c}{ Supplier } & \multicolumn{1}{c}{ Catalog number } \\
\hline Scrambled negative control & Integrated DNA Technologies & 51-01-19-09 \\
shRNA-human GAA & GE Dharmacon & RHS3979-9616754 \\
shRNA-human UQCRC1 & GE Dharmacon & RHS3979-200800674 \\
shRNA-human NDUFS3 & GE Dharmacon & RHS3979-201765752 \\
shRNA-human CTSB & GE Dharmacon & RHS3979-201735931 \\
\hline
\end{tabular}

\subsubsection{Primers}

The sequence of the primers used for qPCR was found in Primer Bank repository from Harvard Medical School and bought from Integrated DNA Technologies. The primers are listed below.

Table 7. Human primers

\begin{tabular}{lll}
\hline Primer & Sequence Forward & Sequence Reverse \\
\hline \hline ACAT2 & CTTTAGCACGGATAGTTTCCTGC & GCTGCAAAGGCTTCATTGATTTC \\
DHCR7 & GCTGCAAAATCGCAACCCAA & GCTCGCCAGTGAAAACCAGT \\
GAPDH & GGAGTCAACGGATTTGGTCG & GACAAGCTTCCCGTTCTCAG \\
HMGCR & TGATTGACCTTTCCAGAGCAAG & CTAAAATTGCCATTCCACGAGC \\
HMGCS1 & GATGTGGGAATTGTTGCCCTT & ATTGTCTCTGTTCCAACTTCCAG \\
HPRT & ACCAGTCAACAGGGGACATAA & CTTCGTGGGGTCCTTTTCACC \\
MVK & CATGGCAAGGTAGCACTG & GATACCAATGTTGGGTAAGCTGA \\
PMVK & CCTTTCGGAAGGACATGATCC & TCTCCGTGTGTCACTCACCA \\
RPL7 & AAGATCAAGCGCCTGAGAAAG & TGCAGGTACATAGAAGTTGCC \\
SCAP & GTGTCTGCAAGTGACCGACC & GATGTCAGGATCAGCATGGAAG \\
\hline
\end{tabular}


Table 8. Mouse primers

\begin{tabular}{lll}
\hline Primer & Sequence Forward & Sequence Reverse \\
\hline \hline ACAT2 & CCCGTGGTCATCGTCTCAG & GGACAGGGCACCATTGAAGG \\
DHCR7 & AGGCTGGATCTCAAGGACAAT & GCCAGACTAGCATGGCCTG \\
GAPDH & TGTGTCCGTCGTTCTGA & CCTGCTTCACCACCTTCTTGA \\
HMGCR & TGTTCACCGGCAACAACAAGA & CCGCGTTATCGTCAGGATGA \\
HMGCS1 & CGgATCGTGAAGACATCAACTC & CGCCCAATGCAATCATAGGAA \\
HPRT & CCTCCTCAGACCGCTTTTT & AACCTGGTTCATCATCGCTAA \\
MVK & GGTGTGGTCGGAACTTCCC & CCTTGAGCGGGTTGGAGAC \\
PMVK & AAAATCCGGGAAGGACTTCGT & AGAGCACAGATGTTACCTCCA \\
RPL7 & CTGCTGGGCCAAAAACTCTCA & CCTTCAACTCTGCGAAATTCCTT \\
RPS12 & CTCATCCACGATGGCCTAGC & ACATGGGCTCATCACAGTTGG \\
SCAP & CCGAGCATTCCAACTGGTG & CCATGTTCGGGAAGTAGGCT \\
SQLE & ATAAGAAATGCGGGGATGTCAC & ATATCCGAGAAGGCAGCGAAC \\
\hline
\end{tabular}




\subsubsection{Antibodies}

The list of antibodies used during this thesis are listed below

Table 9. Primary antibodies for immunoblotting

\begin{tabular}{|c|c|c|c|}
\hline Antibody & Supplier & Catalog number & Dilution \\
\hline ACC & Cell signaling & 3676 & $1: 1000$ \\
\hline ACC pSer79 & Cell signaling & 3661 & $1: 1000$ \\
\hline ATG5 & Cell signaling & 12994 & $1: 1000$ \\
\hline P70S6K1 & Cell signaling & 2708 & $1: 1000$ \\
\hline $\begin{array}{l}\text { P70S6K1 } \\
\text { pThr389 }\end{array}$ & Cell signaling & 9234 & $1: 1000$ \\
\hline TSC2 & Cell signaling & 4308 & $1: 1000$ \\
\hline pTSC2 & Cell signaling & 5584 & $1: 1000$ \\
\hline GAPDH & Sigma Aldrich & G9545 & $1: 10000$ \\
\hline HPRT & Abcam & ab10479 & $1: 4000$ \\
\hline LC3B (D11) & Cell Signaling & 3868 & $1: 2000$ \\
\hline S6 & Cell signaling & 2217 & $1: 2000$ \\
\hline S6 pSer235/236 & Cell signaling & 4858 & $1: 2000$ \\
\hline UQCRC1 & Abcam & ab110252 & $1: 1000$ \\
\hline SREBP1 & Novus & NB100-2215 & $1: 500$ \\
\hline SREBP2 & Abcam & ab30682 & $1: 500$ \\
\hline HMGCR & Abcam & ab174830 & 1:1000 \\
\hline HMGCR Ser872 & BIOSS & bs4063R & $1: 1000$ \\
\hline AMPK $\alpha$ & Cell signaling & 5832 & $1: 1000$ \\
\hline pAMPK $\alpha$ & Cell signaling & $2535 S$ & 1:1000 \\
\hline
\end{tabular}


Table 10. Secondary antibodies for immunoblotting

\begin{tabular}{lcc}
\hline \multicolumn{1}{c}{ Antibody } & Supplier & Code \\
\hline \hline Goat anti-mouse IgG & Dianova & $115-035-146$ \\
Goat anti-rabbit IgG & Dianova & $115-035-144$ \\
\hline
\end{tabular}

\subsubsection{Instruments}

The instruments used for this thesis are listed below

Table 11. List of instruments and equipment

\begin{tabular}{ll}
\multicolumn{1}{c}{ Instrument } & \multicolumn{1}{c}{ Manufacturer } \\
\hline \hline Ace Block Digital Dry Bath & Labnet \\
Bio-Rad Power Pack HC Mini-Protean Tetra & Bio-Rad \\
System & \\
Centrifuge 5415R & Eppendorf \\
Centrifuge 5810R & Eppendorf \\
Centrifuge Allegra X-15R & Beckman Coulter \\
Countess C10281 & Invitrogen \\
Cryogrider tool kit 230V & OPS Diagnostics \\
Gene Quant 1300 & GE Healthcare \\
Hood Herasafe & Thermo Scientific \\
Incubator Heracell 150i & Thermo Scientific \\
Leica CM 1850 Cryostat & Leica Biosystems \\
Multichannel pipette & Eppendorf Research \\
Nanodrop 2000C & Peqlab \\
pH meter pH7110 & WTW Inolab \\
Pipette gun accu-jet pro & OHAUS \\
Pipettes & Ependorf Research \\
Potter S (Dounce homogenizer) & Scientific Inc. \\
\hline
\end{tabular}




\begin{tabular}{ll}
\hline Quant Studio 6 Flex & Life Technologies \\
Revolver wheel & Labnet \\
Scanner Epson Perfection V850 Pro & Epson \\
SE600 Ruby system & GE Healthcare \\
SYNERGYM1 microplate reader & BioTek \\
Thermocycler UNO II & Biometra \\
Vortex RS-VA10 & Phoenix Instrument \\
\hline
\end{tabular}

\subsubsection{Software}

The programs used during this project are listed below:

Adobe Illustrator CS6, Adobe Systems Inc., USA

GraphPad Prism 7, GraphPad Software Inc., USA

ImageJ, NIH, USA

Ingenuity Pathway Analysis, QIAGEN, USA

Mendeley, Mendeley Ltd, UK

Microsoft Office, Microsoft Corporation, USA

Strand NGS, Strand Life Sciences, India 


\subsection{Methods}

\subsubsection{Cell Culture}

\subsubsection{Procedure for plating cells}

All cells were grown in Dulbecco's Modified Eagle Medium high glucose medium (DMEM) supplemented with $10 \%$ fetal bovine serum (FBS) and $1 \%$ Penicillin/Streptomycin (P/S), hereafter called normal medium, in a monolayer cultured at $37^{\circ} \mathrm{C}$ and $5 \% \mathrm{CO}_{2}$. Only UQCRC1 KD HeLa cells and their corresponding scrambled controls were grown in normal medium supplemented with $1 \mathrm{mM}$ pyruvate. Prior use, all media, and solutions used for cell culture were either autoclaved or filtered. Cells were counted using a cell counter, and they were about $95 \%$ confluent when collected for experiments. $24 \mathrm{~h}$, before realizing the experiment, cells were plated, and the seeding cell number was modified according to the area of the plate. The most used seeding densities during this project were approximate:

\begin{tabular}{|c|c|}
\hline Size of the cell culture plate & Seeding density \\
\hline $15 \mathrm{~cm}$ & $3.0 \times 10^{6}$ cells \\
\hline $10 \mathrm{~cm}$ & $1.25 \times 10^{6}$ cells \\
\hline $6 \mathrm{~cm}$ & $3.0 \times 10^{5}$ cells \\
\hline 6-well plate & $1.5 \times 10^{5}$ cells \\
\hline 96-well plate & $1.5 \times 10^{4}$ cells \\
\hline
\end{tabular}

\subsubsection{Collection of cells}

The cells were collected for the preparation of the whole-cell lysates for nucleic acid, protein, and lipid extraction by removing the growth medium and washing one time in cold PBS. For this process, the medium was replaced with $5 \mathrm{ml}$ cold PBS, and cells were scraped on ice. After obtaining the suspension of cells in PBS, the suspension was divided into two: $500 \mu$ l were collected in an Eppendorf tube (this fraction was used for nucleic acid isolation), and the rest was collected in a falcon latter used for protein extraction or lipid extraction. The cell suspensions were pelleted at $3600 \mathrm{rpm}, 4^{\circ} \mathrm{C}$ for 5 minutes. The supernatant was discarded, and the pellets were used immediately for the experiments or stored at $-20^{\circ} \mathrm{C}$ for later use.

\subsubsection{Mitochondrial isolation from cultured cells}


Mitochondria were isolated from cultured cells according to a previously described protocol (Wieckowski et al., 2015). Cells were collected by incubation for 5 minutes with trypsin, and trypsinization was halted by the addition of fresh normal medium. The cell suspension was then centrifuged for 5 minutes at $600 \mathrm{~g}, 4^{\circ} \mathrm{C}$. The pellet obtained from the centrifugation was washed with PBS and centrifuged again as previously described. The supernatant obtained from the centrifugation was discarded, the pellet was resuspended in homogenization buffer (mitochondrial isolation buffer, previously described, supplemented with protease and phosphatase inhibitors, $1 \mathrm{mM}$ DTT, and $100 \mu \mathrm{M}$ PMSF) and incubated for 15 minutes on ice. To avoid degradation of the sample, further steps of the isolation were carried out at $4^{\circ} \mathrm{C}$.

After the incubation on ice, the cells were homogenized using a Potter-Evehjem homogenizer with a Teflon pestle for at least 50 strokes. The homogenate was transferred to a new polypropylene centrifuge tube and centrifuged for 5 minutes at $600 \mathrm{~g}, 4^{\circ} \mathrm{C}$. The obtained pellet was discarded (unbroken cells and nuclei) and the supernatant was collected into a new tube for another centrifugation step of 5 minutes at $600 \mathrm{~g}, 4^{\circ} \mathrm{C}$. Again, the pellet was discarded, and the new supernatant was centrifuged 10 minutes at $7000 \mathrm{~g}, 4^{\circ} \mathrm{C}$. The mitochondrial fraction(pellet), was resuspended in 5-10 $\mathrm{ml}$ homogenization buffer (according to the size of the pellet) and centrifugated again for 10 minutes at $7000 \mathrm{~g}, 4^{\circ} \mathrm{C}$; this step was repeated twice. Finally, the pellet was resuspended in $200 \mu \mathrm{L}$ of homogenization buffer.

\subsubsection{Pharmacological treatments}

Before treatment, HeLa cell and mouse embryonic fibroblast (MEF) cell were seeded according to de densities previously described. The concentrations used (unless stated otherwise) were: $250 \mu \mathrm{M}$ Rotenone, $10 \mu \mathrm{M}$ U18666A, and $250 \mathrm{nM}$ Torin. For the experiment with supplementation of $1 \% \mathrm{v} / \mathrm{w}$ (2-Hydroxypropyl)- $\beta$-cyclodextrin (HPCD), the compound was previously dissolved in normal medium and filtered to avoid contamination. Before the addition of the drug to the medium, cells were washed with PBS, and the normal medium was replaced with medium supplemented with the corresponding drug or its control.

\subsubsection{Generation of stable HeLa KDs}

The generation of stable knockdown in HeLa cells were generated using shRNA following the protocol described by (Fernandez-Mosquera et al., 2019). Briefly, lentiviral stable knock downs were generated by transfecting HEK293T packaging cells with an optimized mix of packaging plasmids and either of GAA, CTSB, and UQCRC1 shRNAs targeting five different regions of each coding gene or scrambled non-targeting negative control shRNA using Lipofectamine 2000. After that, lentiviral vectors were concentrated using the Lenti-X concentrator. HeLa cells were transduced with lentiviral particles supplemented with $8 \mathrm{ug} / \mathrm{ml}$ Polybrene. For the selection of the knockdowns, puromycin was used. Finally, the efficiency of the knockdown was confirmed by western blot and qPCR. 


\subsubsection{Mice handling}

\subsubsection{Mouse lines}

The mouse lines used for the experiments, both obtained from Jackson's Lab USA, were Gaa KO mice and Ndufs $4 \mathrm{KO}$ mice, and their wild-type controls. All the procedures concerning the animals were reviewed and approved by the Institutional Animal Care and Use Committee of the University Medical Center Göttingen.

\subsubsection{Mouse tissue extraction}

Before euthanizing mice by cervical dislocation, the mice were anesthetized with isoflurane. For the extraction of tissue, the body of the animal was placed in a petri dish and sprayed with $70 \%$ ethanol. Once the abdomen was opened, a small piece of the liver was cut and deposited in an Eppendorf tube. For heart extraction, a similar procedure was followed. The heart was rinsed in PBS several times to remove the blood and placed in an Eppendorf tube. Skeletal muscle (quadriceps) was collected from the mice after removing the skin and placed in an Eppendorf tube. Immediately after each sample was collected, they were placed in a container with liquid nitrogen for a few seconds. After the period in liquid nitrogen, samples were stored at $-80^{\circ} \mathrm{C}$. Before usage for other applications, tissues were powdered by grinding under liquid nitrogen using a cryogrinder toolkit. The powder was split into several tubes for further experiments and stored at $-80^{\circ} \mathrm{C}$.

\subsubsection{Molecular biology}

\subsubsection{RNA isolation}

\subsection{Mouse tissue}

For the isolating RNA from tissues, TRI reagent (Sigma) was used. $1 \mathrm{ml}$ of TRI reagent was added to the powder of the tissues (previously generated) in a $1.5 \mathrm{ml}$ Eppendorf tube to form a homogenate. This homogenate was incubated for 5 minutes at room temperature. After the incubation, $200 \mu \mathrm{L}$ of chloroform was added. To mix the sample, the tubes were shaken vigorously by hand for 15 seconds and incubated at for 2-3 minutes at RT. The samples were then centrifuged at $12000 \times \mathrm{g}$ for 15 minutes at $4^{\circ} \mathrm{C}$. Approximately $500 \mu \mathrm{L}$ of the aqueous phase was carefully removed and placed in new RNAse-free $1.5 \mathrm{~mL}$ Eppendorf tubes. For the RNA precipitation isopropanol was used, in this case, the precipitation was done by adding $500 \mu \mathrm{L}$ of isopropanol and incubating at room temperature for 10 minutes. After 10 minutes, the samples were centrifuged at $12000 \mathrm{~g}$ for 10 minutes at $4^{\circ} \mathrm{C}$. The RNA pellet was washed in $1 \mathrm{~mL} 75 \%$ ethanol, vortexed briefly and centrifuged at $7500 \mathrm{~g}$ for 5 minutes at $4^{\circ} \mathrm{C}$. The supernatant was completely removed and the RNA pellet was dried for 5-10 minutes at RT. Finally, the pellet was resuspended in $300 \mu \mathrm{L}$ of RNAse-free water. The RNA concentration was determined and the RNA was either used immediately or frozen at $-80^{\circ} \mathrm{C}$. 


\subsection{Cultured Cells}

For RNA isolation from cells, the commercial kit CRYSTAL RNA Mini Kit (BIOLAB) was used. The procedure was done according to the manufacturer's specification. Cell pellets were incubated with $400 \mu \mathrm{L}$ of Lysis Solution RL (supplemented with 1\% 2-Mercaptoethanol $(\beta-\mathrm{SH})$ ). The initial incubation period was 2 minutes, after that the pellet was resuspended and incubated again for 3 minutes at room temperature. After the incubation, the suspension was transferred into a Spin Filter D column set ina $2 \mathrm{~mL}$ Receiver Tubes and centrifuged for 2 minutes at $10000 \mathrm{~g}$. After the centrifugation, the Spin Filter D was discarded and $400 \mu \mathrm{L}$ of $70 \%$ ethanol was added to the flowthrough. Once the ethanol and the sample were mixed by pipetting, they were transferred to Spin Filter R in a new Receiver Tubes $2 \mathrm{~mL}$. The Spin Filter R was centrifuged for 2 minutes at $10000 \times \mathrm{g}$. After the centrifugation, the Spin Filter R was placed into a new tube. In this new tube, $500 \mu \mathrm{L}$ of Washing Solution HS was added to the Spin Filter, and the column was centrifuged for 1 minute at $10000 \mathrm{~g}$. For the last wash, $700 \mu \mathrm{L}$ of Washing Solution LS was added to the Spin Filter $\mathrm{R}$ and centrifuged as before for 1 minute. To dry the membrane of the Spin Filter $\mathrm{R}$, the column was placed into a clean $2 \mathrm{~mL}$ receiver tube and centrifuged for 2 minutes at $10000 \mathrm{~g}$. Finally, for the elution of RNA, the column was incubated 1 minute with RNAase-free water and centrifuged for 1 minute at $6000 \times \mathrm{g}$ to collect the RNA.

\subsubsection{Determination of nucleic acid concentrations}

To measure RNA concentration, The Nanodrop (PeqLab) spectrophotometer was used. For this purpose, $1 \mu \mathrm{L}$ of blank (nuclease-free water) or sample was applied to the Nanodrop. The Nanodrop measured the sample concentration, and absorbances at $260 \mathrm{~nm}$ and $280 \mathrm{~nm}$ to control the quality of nucleic acids. The results of RNA concentration were then exported as Microsoft Excel files.

\subsubsection{Synthesis of cDNA}

Complementary DNA (cDNA) was employed for the experiments of gene expression experiments. the isolated RNA (isolation previously described) was reverse transcribed using iScript cDNA synthesis kit. All the reagents of the kit and the sample were kept on ice during the whole process. The reaction in each PCR tube was made of the following components: $1 \mu \mathrm{g}$ of RNA, $4 \mu \mathrm{L}$ of $5 \mathrm{X}$ iScript reaction mix, $1 \mu \mathrm{L}$ of iScript reverse transcriptase, and nuclease-free water to reach the final volume of $20 \mu \mathrm{L}$. Once the PCR tubes was vortexed, they were centrifuged shortly. Finally, the tubes were incubated in a thermocycler with the following protocol: priming for 5 minutes at $25^{\circ} \mathrm{C}$, reverse transcription for 30 minutes at $46^{\circ} \mathrm{C}$, reverse transcriptase inactivation for 1 minute at $95^{\circ} \mathrm{C}$, and the last step of holding at $4^{\circ} \mathrm{C}$. The synthesized cDNA stored at $-20^{\circ} \mathrm{C}$ until it was used in qPCR.

\subsubsection{Quantitative-PCR (qPCR)}

Real-time PCR was employed to measure the levels of transcripts from the previously synthesized cDNA. Real-time PCR was performed in at least four technical replicates using Luna ${ }^{\circledR}$ Universal 
qPCR Master Mix. The final volume of the reaction was $8 \mu \mathrm{L}$ per well in a 384-well plate, which consisted of $3.6 \mu \mathrm{L}$ Luna ${ }^{\circledR}$ Universal qPCR Master Mix, $0.4 \mu \mathrm{l}$ of primers ( $0.2 \mu \mathrm{L}$ of forward and $0.2 \mu \mathrm{L}$ of reverse) for each gene, and $4 \mu \mathrm{l}$ of diluted cDNA template (the dilution used was 1:50). The plate was sealed with adhesive seal, shortly centrifuged for 1 minute and incubated in the QuantStudio $^{\text {TM }} 6$ Flex Real-Time PCR system (Life Technologies). The following protocol was used for qPCR: polymerase activation for 30 seconds at $95^{\circ} \mathrm{C}$, amplification for 40 cycles with steps of denaturation for 3 seconds at $95^{\circ} \mathrm{C}$, annealing for 30 seconds at $56^{\circ} \mathrm{C}$, and extension for 30 seconds at $60^{\circ} \mathrm{C}$. Melt-curve analyses were done at steps of $85^{\circ} \mathrm{C}$ for 15 seconds, $52^{\circ} \mathrm{C}$ for 30 seconds and $95^{\circ} \mathrm{C}$ for 15 seconds. Data obtained were analyzed with QuantStudio ${ }^{\mathrm{TM}}$ Real-Time PCR Software and exported as Microsoft Excel files for further analyses using the $\Delta \triangle C T$ method.

\subsubsection{Biochemical experiments}

\subsubsection{Preparation of protein samples from mouse}

\subsection{Protein extraction from tissue powder}

For the preparation of tissue lysates, the tissue powder previously obtained was resuspended in $500 \mu \mathrm{L}$ of RIPA buffer supplemented with protease and phosphatase inhibitor to avoid protein degradation. The tube was vortexed briefly, and the tissue was lysed by rotation at $4^{\circ} \mathrm{C}$ for 1 hour. Tissue homogenate were centrifuged at $16000 \mathrm{rpm}, 4^{\circ} \mathrm{C}$ for 20 minutes. The resulting supernatant (tissue lysate) was collected into a new $1.5 \mathrm{~mL}$ Eppendorf tube. The protein concentration of the tissue lysate was determined by the Pierce Assay. Lysates were used for further experiments or stored at $-80^{\circ} \mathrm{C}$.

\subsection{Protein concentration determination of Tissue lysates}

To determine the protein concentration of tissue lysates, the Pierce BCA Protein Assay Kit (BioRad) was used, the manufacturer's instructions were followed for the protein quantification. The protein concentration of the lysates was determined in duplicates and before the quantification samples were diluted in a proportion of 1:40. For the quantification, a calibration curve was made of BSA standard. $50 \mu \mathrm{L}$ of diluted samples were mixed with $1 \mathrm{~mL}$ Pierce BCA working solution (reagents $A$ and $B, 50: 1$ ) in a glass tube and vortexed briefly. The tubes were incubated at $37^{\circ} \mathrm{C}$ for 30 minutes in the dark and transferred into plastic cuvettes. The absorbance was measured at $562 \mathrm{~nm}$ using a GeneQuant 1300 spectrophotometer. Finally, the protein concentration of the lysates was determined based on the standard calibration curve using Microsoft Excel software.

\subsubsection{Preparation of proteins from cells}

\subsubsection{Protein extraction from pellets}

The pellets obtained from cells, were placed on ice and about 50 to $100 \mu \mathrm{L}$ of whole cell extraction buffer (see Table 3 above) was added depending on the pellet size. The suspension was transferred to $1.5 \mathrm{ml}$ Eppendorf tubes and lysed by rotation at $4^{\circ} \mathrm{C}$ for 30 minutes. The resulting 
cell lysate was then centrifuged at $16000 \mathrm{rpm}, 4^{\circ} \mathrm{C}$ for 20 minutes. The supernatant (containing the whole cell lysate) was collected into new $1.5 \mathrm{~mL}$ Eppendorf tubes. The protein concentration was determined by the Bradford Assay. Lysates were either used immediately or stored at $-20^{\circ} \mathrm{C}$.

\subsection{Protein concentration determination of cell lysates}

Protein concentration from cells was determined using Protein Assay Dye Reagent Concentrate (Bio-Rad). The procedure used was according to the manufacturer's instructions. For standard curve defined concentrations of BSA was used. In order to measure the protein concentration (at least duplicates were measured), $1 \mu \mathrm{L}$ of the sample was diluted in $800 \mu \mathrm{L}$ of ddH2O. $200 \mu \mathrm{L}$ of protein assay dye reagent was added and the tubes were briefly mixed by vortexing and incubated in the dark for 5 minutes at room temperature (RT). After the incubation period, the mix was transferred into plastic cuvettes and the absorbance was measured at $595 \mathrm{~nm}$ using a GeneQuant 1300 spectrophotometer. The protein concentration was calculated based on the standard calibration curve using Microsoft Excel 2013

\subsubsection{SDS-Polyacrylamide gel electrophoresis}

\subsection{Preparation of gels}

In order to separate denatured proteins according to their molecular weight, a polyacrylamide gel was prepared. The acrylamide gels were prepared using the Bio-Rad Gel preparation system and $40 \%$ acrylamide solution. In all experiments, the used concentration of the resolving gel was $12 \%$ (composition previously described), and the thickness of the gel was $1 \mathrm{~mm}$. Initially, the resolving gel was added between two glasses and covered with isopropanol. After the polymerization, the isopropanol was discarded from the gel. The gel was rinsed with $\mathrm{dd}_{\mathrm{d}} \mathrm{H}_{2} \mathrm{O}$. Once the water was drained, the $4 \%$ stacking gel (see Table 3 above) was added on the top of the resolving gel. Immediately after the gel was poured, the 10 -well comb was inserted into the stacking gel. After the polymerization of the gels, the gels were placed in a container filled with $1 \mathrm{X}$ running buffer for storage at $4^{\circ} \mathrm{C}$ or used immediately.

\subsection{Electrophoresis}

The Mini-Protean Tetra System (Bio-Rad) was used to perform the SDS-PAGE. The gels were placed in the running apparatus without the combs, and the container was filled with $1 \mathrm{X}$ running buffer. The samples were prepared by mixing 6x SDS loading buffer and a determined protein concentration of $30 \mu \mathrm{g}$. Once the sample was mixed with the loading buffer, the mix was boiled at $95^{\circ} \mathrm{C}$ for 5 minutes. In all the wells of the gel, the same amount of protein was loaded. The electrophoresis was run at a constant voltage. Initially, the voltage was set at $100 \mathrm{~V}$. When the samples entered the resolving gel, the voltage was changed to $180 \mathrm{~V}$. As a standard of molecular weight, the Page Ruler plus Prestained was used.

\subsubsection{Western blotting}


Western blot was carried according to the protocols described by (Fernandez-Mosquera et al., 2019). Polyvinylidene fluoride membrane (PVDF) were activated for $15 \mathrm{sec}$ in methanol, washed with ddH2O for 1 minute, and equilibrated for $5 \mathrm{~min}$ in transfer buffer prior to use. After the run, the gels were taken out of the glass plate, the stacking gel was cut out, and gels were equilibrated in cold transfer buffer. Before use, the transfer sponges and sheets of Whatman paper were previously soaked for some time in transfer buffer. Using a tank transfer system, the transfer sandwich was assembled on a plastic transfer cassette soaked in transfer buffer to avoid trapping air bubbles. In between two sponges, and two sheets of Whatman paper on each side, the activated membrane was placed on top of the gel. A rolling tool was used throughout to completely remove air bubbles. The assembled transfer cassetes with the sandwich were placed in the transfer chamber and filled with transfer buffer. Finally, a cold ice pack was placed in the tank to avoid excessive heating. The electrophoresis was done at constant voltage 100V for 90 minutes at $4^{\circ} \mathrm{C}$.

\subsubsection{Immunodetection}

Following transference, the membranes were blocked in 5\% Milk in TBST with gentle shaking for 1 hour at RT. After the blocking, membranes were washed three times in TBST for 10 minutes and incubated with primary antibodies overnight at $4{ }^{\circ} \mathrm{C}$. After the incubation with the primary antibody, membranes were washed three times with TBST for 10 minutes. After the washes, the membranes were incubated for $1 \mathrm{~h}$ at room temperature with the corresponding HRP-conjugated secondary antibodies. The secondary antibody was prepared in the in blocking buffer. The secondary antibody was discarded, and the membranes were washed three more times in TBST for 10 minutes. Once done with the washes, the chemiluminescence was detected by incubating membranes in Luminata Western HRP substrate during 3 minutes, and developing signals on medical X-ray films using the AGFA Curix 60 processor. For quantification, the films were scanned, and quantification of the bands was done using ImageJ software.

\subsubsection{Cholesterol content measurement}

\subsubsection{Free cholesterol content measurement by Filipin}

Cells were seeded in a 96-well plate (seeding density previously described) the day before the experiment. Initially, the cells were washed three times for 10 minutes with ice-cold PBS. Once the washes were done, the filipin was added (concentration of $0.1 \mathrm{mg} / \mathrm{mL}$ dissolved in PBS) to each well and incubated during 30 minutes in dark conditions with mild shaking. After the incubation, cells were washed three times with ice-cold PBS. The measurement of the fluorescent intensity was done in a microplate reader, and the content of cholesterol was normalized to protein content using Pierce BCA microassay.

\subsubsection{Protein concentration determination using Pierce BCA microassay}


Once the cholesterol content was measured with filipin in 96-well plates, the protein concentration per well of samples was quantified for normalization of the cholesterol content. For this purpose, immediately after the measurement with filipin, PBS was removed, and the cells were lysed with $125 \mu \mathrm{L}$ of $\mathrm{dd}_{2} \mathrm{H}_{2} \mathrm{O}$ for 60 minutes with mild shaking. After the lysis, protein concentrations were determined using the Pierce BCA Protein Assay kit. To measure the protein concentration, $100 \mu$ of double concentrated Pierce BCA working reagent (reagents A and B, 25:1) was added to each well-containing sample and blank. Samples were mixed and incubated for 30 minutes at $37^{\circ} \mathrm{C}$. Finally, the absorbance was measured at $562 \mathrm{~nm}$ in a microplate reader. The protein concentration was calculated from a standard curve made from albumin standards.

\subsubsection{Lipids extraction}

The lipid extraction was done according to the extraction procedure described by Folch and colleagues (Folch et al., 1957). The extraction of lipids was done from harvested cells and isolated mitochondrial fractions. In the case of extraction from harvested cells, they were collected with $5 \mathrm{~mL}$ PBS (50 $\mu \mathrm{L}$ of the sample was kept for protein quantification by Bradford assay) and after centrifugation for 5 minutes at $3600 \mathrm{rpm}, 4^{\circ} \mathrm{C}$. The obtained pellet or $100 \mu \mathrm{L}$ of the isolated mitochondrial fraction were resuspended in $250 \mu \mathrm{L}$ of a mix made of chloroform/methanol (2/1). Immediately, the cell suspension was transferred to a glass tube and vortexed briefly. The solvent was washed with 0.2 volumes of $\mathrm{dd}_{2} \mathrm{O}$, in this case, $50 \mu \mathrm{L}$ was used. After vortexing for a few seconds, the suspension was centrifuged for 5 minutes at $2000 \mathrm{rpm}$ to separate the two phases. The lower chloroform phase contains the lipids, and this phase was collected, taking $100 \mu \mathrm{L}$ directly from the bottom of the tube. The chloroform was evaporated under vacuum for at least 30 minutes and the lipids resuspended in "Reaction Buffer" provided from Amplex ${ }^{\circledR}$ Red Cholesterol Assay Kit.

\subsubsection{Cholesterol quantification using Amplex ${ }^{\circledR}$ Red Cholesterol Assay Kit}

Total cholesterol content was determined using the Amplex ${ }^{\circledR}$ Red Cholesterol Assay Kit, the procedure according to the manufacturer specifications was followed. The experiments were done in a 96-well plate and with at least technical triplicates. To prepare the samples, to each well $5 \mu \mathrm{L}$ of the previously extracted lipid samples was added to $45 \mu \mathrm{L}$ of Reaction Buffer. To start the reaction, $50 \mu \mathrm{L}$ of Amplex Red working solution was added (working solution contains 300 $\mu \mathrm{M}$ Amplex ${ }^{\circledR}$ Red reagent, $2 \mathrm{U} / \mathrm{mL} \mathrm{HRP}, 2 \mathrm{U} / \mathrm{mL}$ cholesterol oxidase, and $0.2 \mathrm{U} / \mathrm{mL}$ cholesterol esterase) and the microplate was incubated during 30 minutes protected from the light. The fluorescence corresponding to cholesterol content was measured at excitation and emission wavelengths of $530 \mathrm{~nm}$ and $590 \mathrm{~nm}$ respectively using, a microplate reader. The cholesterol content was calculated from a standard curve made from cholesterol and then normalized to the protein concentration of each sample.

\subsubsection{Statistical analyses and graphs}

For statistical analysis, Microsoft Excel 2016 was used. Graphs were plotted with GraphPad Prism version 7. In all cases, the graphs show mean normalized to loading control (western blot) or 
control gene (qPCR). The mean of controls was always fixed to 1 . The error bars correspond to standard deviation or standard error of the mean for at least biological triplicates as stated in the legends for each figure. The differences between the means were determined by the Student's t-test for two-parameter comparisons. These differences were displayed in the graphs as * $\mathrm{p}<$ $0.05 ; * * p<0.01 ; * * * p 0.001$.

\subsubsection{Bioinformatic analysis}

RNA sequencing was performed following the protocol described by Murdoch et al., 2016. The pathway analysis was done as described in Raimundo et al., 2012. Briefly, RNA samples were purified from NDUFS3, UQCRC1, GAA, and CTSB knock down HeLa cell lines along with scrambled controls using CRYSTAL RNA mini kit. The RNA concentration and quality were determined by Nanodrop. Samples were sequenced on HiSeq 2000 (Illumina) with support from the Transcriptome and Genome Analysis Laboratory (TAL) of the University of Goettingen Medical Center (UMG).

Read alignment and quality assessment, as well as normalization, were done using Strand NGS software. Differentially expressed genes in the respective knock downs relative to the scrambled control with adjusted $p$-value less than 0.05 were imported into the Ingenuity Pathway Analysis (IPA) software for functional enrichment analysis. In the result section, the top ten significantly altered (Fischer exact test $<0.05$ ) pathways are displayed. 


\section{RESULTS}

\subsection{Generation of the stable knock downs}

Initially, we generated stable knock downs (KDs) of our genes of interest using short hairpin RNAs (shRNAs) delivered by lentiviral vectors to HeLa cells. We selected two mitochondrial and two lysosomal genes. In the case of the mitochondrial genes, we selected NADH:Ubiquinone Oxidoreductase Core Subunit S3 (NDUFS3, a subunit of the complex I of the respiratory chain) and Ubiquinol-Cytochrome C Reductase Core Protein I (UQCRC1, a subunit of the complex III of the respiratory chain). From the lysosomal genes, we selected Cathepsin B (CTSB) and Acid Alpha Glucosidase (GAA) both involved in the macromolecules in the lysosome. In all cases, the protein selected has been studied as different diseases models. For the mitochondrial models: NDUFS3 deficient cells are a mitochondrial complex I deficiency model (Haack et al., 2012), UQCRC1 is studied as a model of chronic mitochondrial dysfunction (Fernandez-Mosquera et al., 2019). In the case of the lysosomal models: cells lacking GAA are Pompe's disease model (Parenti et al., 2015), and CTSB is enzymes with huge importance in cancer (Olson and Joyce, 2015). KDs of these four genes were generated as described above, thus creating two model cell lines of mitochondrial dysfunction, and two of lysosomal dysfunction.

A

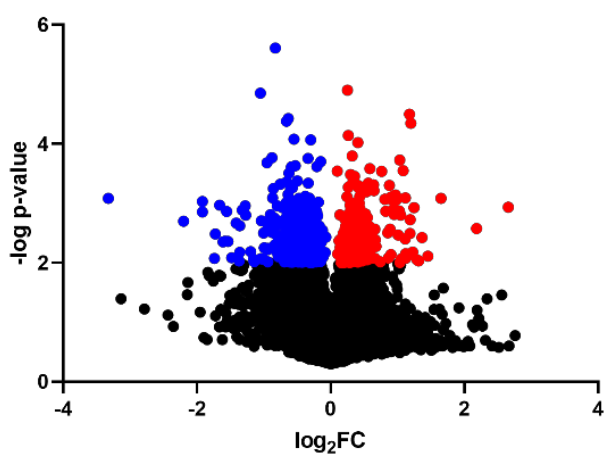

C

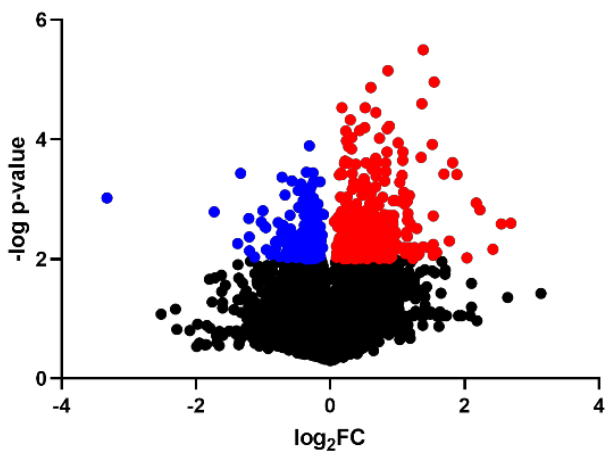

B

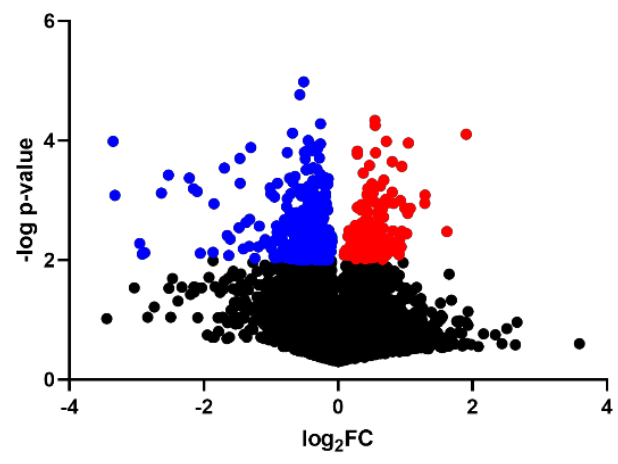

D

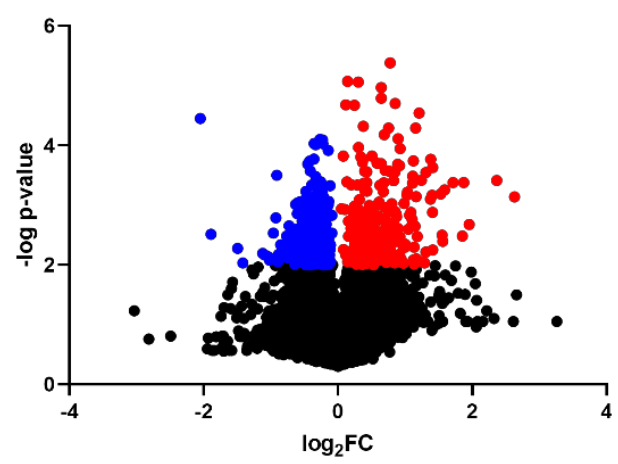


Figure 12. Transcriptome data analyses. Volcano plots corresponding to NDUFS3 KD (A), UQCRC1 KD (B), GAA KD (C) and CTSB KD (D). Downregulated genes are shown in blue, upregulated genes in red and unchanged genes in black. Differentially expressed genes were identified by a cut-off of adjusted $p$-value $<0.01$.

We tested five different shRNAs for each gene and determined the two most efficient ones for each target. Once we confirmed the individual KDs at both transcript and protein levels, RNA was extracted from all the cell lines (UQRCR1 KD, NDUFS3 KD, CTSB KD, and GAA KD) and a control cell (scrambled shRNA). Next, the RNA was sent to the next generation sequencing (NGS) facility available in Göttingen for RNA sequencing (RNA-Seq). The data that we obtained from the RNASeq was analyzed in the lab using the most recent bioinformatic tools available comparing the expression levels of the genes in each stable KD with the control cell line., in this way we obtained a differentially regulated gene list.

\section{Lysosome KDs}

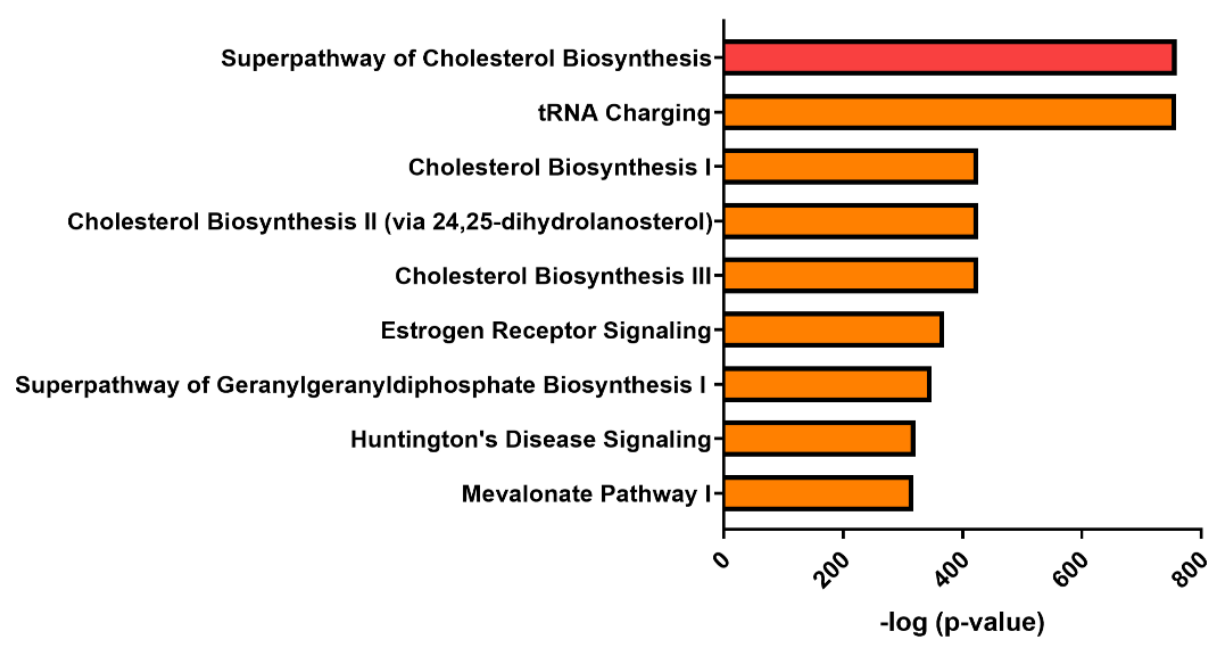

Mitochondria KDs

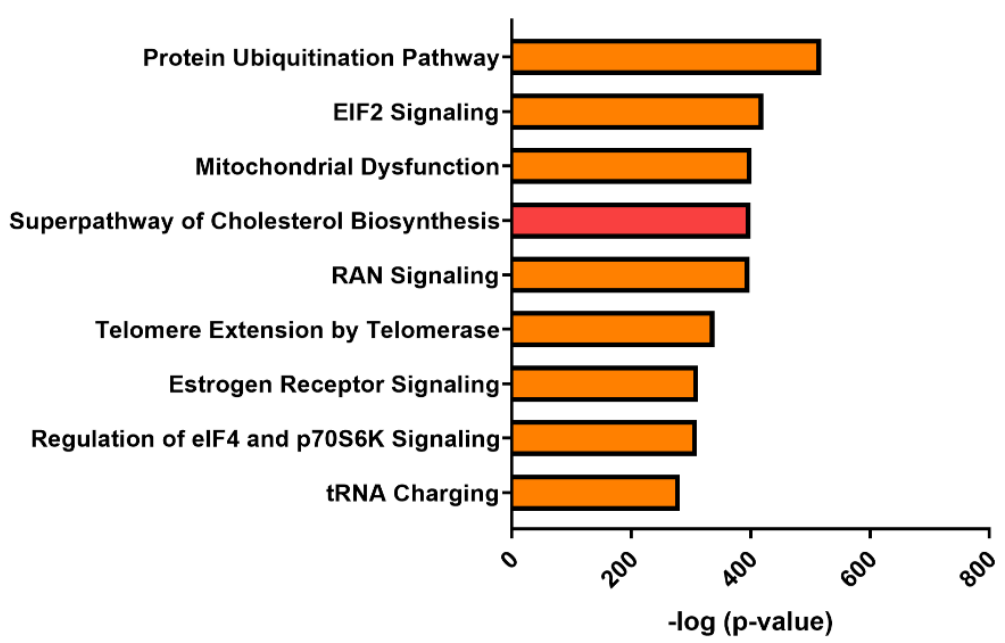


Figure 13. Top 10 canonical pathways affected in each model. Top panel: Canonical pathways altered in lysosomal KDs. Bottom panel: Canonical pathways impaired in mitochondrial KDs. Superpathway of cholesterol biosynthesis is marked in red. Associated $\mathrm{P}$ values were determined according to analysis in the database for annotation, visualization, and integrated discovery (Fisher exact P-value).

The analyzed data showed KD-specific differentially expressed genes (Fig. 12), which we fed through our multi-dimensional genomics analyses pipeline. One of the analyzed dimensions is the pathways enriched in our differentially regulated gene list. The top pathways perturbed by all the KDs were lipid biosynthetic pathways, particularly the cholesterol pathway (Fig. 13). Cholesterol is an essential lipid in the cell membrane, controlling and contributing to its fluidity (Goldstein et al., 2006).
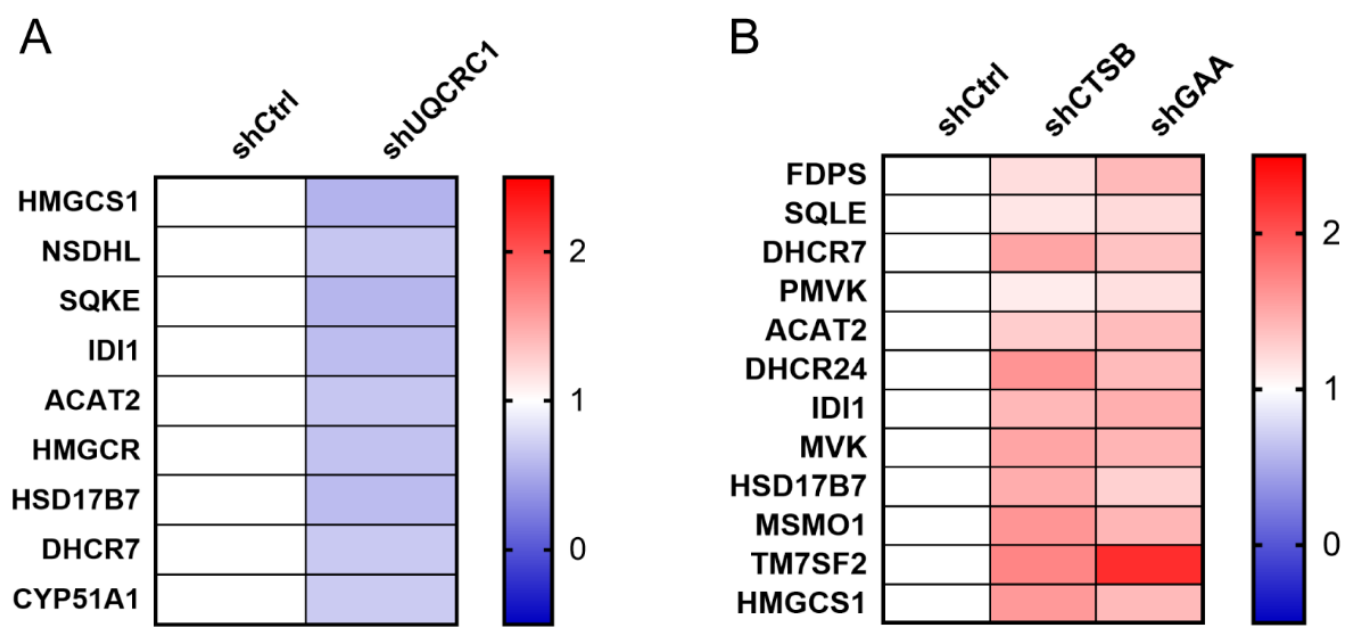

Figure 14. Transcriptome data analyses. The selected transcripts, represented in the heatmaps, were obtained from the canonical pathway of cholesterol biosynthesis significantly change in the pathway analysis. Heatmaps, showing reduced transcript levels of enzymes necessaries for the cholesterol biosynthesis in UQCRC1 KD (A) and increased in the transcript levels of cholesterol biosynthesis in GAA and CTSB KDs (B).

The KD of one key mitochondrial protein, UQCRC1 KD resulted in a downregulation of $40 \%$ of the transcripts involved in the cholesterol biosynthesis pathway (Fig. 14A). On the other hand, lysosomal perturbations (CTSB and GAA KDs) triggered an increase of approximately $45 \%$ in the transcript levels of the genes from the cholesterol biosynthesis pathway. This increase suggests an activation of the cholesterol biosynthesis pathway (Fig. 14B).

Next, we aimed to validate using quantitative real-time PCR (qPCR) the transcript levels for our genes of interest.

\subsection{Validation of the data obtained from the analyses of the RNA Seq results}

\subsubsection{Mitochondrial dysfunction model}


We validated by qPCR the results obtained from the transcriptomic analyses by evaluating the expression levels of the key genes of the enzymes involved in the cholesterol biosynthesis pathway. The main enzymes selected were Acetoacetyl-CoA Synthetase (ACAT2), HMG-CoA Synthase (HMGCS1), HMG-CoA Reductase (HMGCR), Mevalonate Kinase (MVK), Phosphomevalonate Kinase (PMVK) and 7-Dehydrocholesterol Reductase (DHCR7).

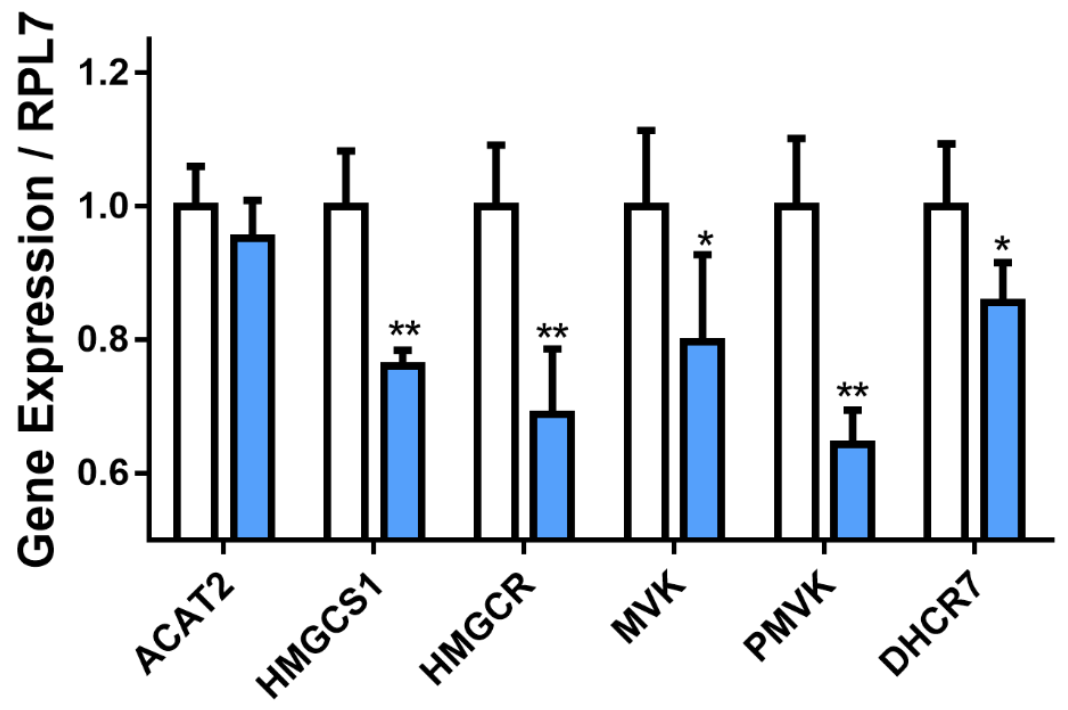

$\square$ SCRAMBLED $\square$ UQCRC1 KD

Figure 15. Validation of RNA Seq results in HeLa UQCRC 1 KD. Expression levels of the main enzymes involved in the cholesterol biosynthesis pathway. The transcript levels were determined by the $\Delta \Delta C T$ method using RPL7 (Ribosomal Protein L7) as a reference gene; the plotted value corresponds to the relative expression of the gene (fold change). Samples were centered to one by normalization of the gene expression of the experimental sample to its corresponding control, in this case, scrambled. Graphs show mean \pm SD; $p<0.05\left({ }^{*}\right), p<0.01(* *)$; Student's t-test; SD: standard deviation. $\mathrm{n}=5$.

As expected, at transcript levels of all the enzymes previously mentioned except ACAT2 were at least between $20-30 \%$ significant decreased. The results we obtained from UQCRC1 KDs were consistent with the outcomes of the NGS data. Indeed, the cholesterol biosynthesis pathway was downregulated in the HeLa UQCRC1 KD (Fig. 15).

\subsubsection{Models of lysosomal dysfunction}

A similar strategy was employed in the case of the models of lysosomal dysfunction that we generated, HeLa CTSB KD and HeLa GAA KD. 

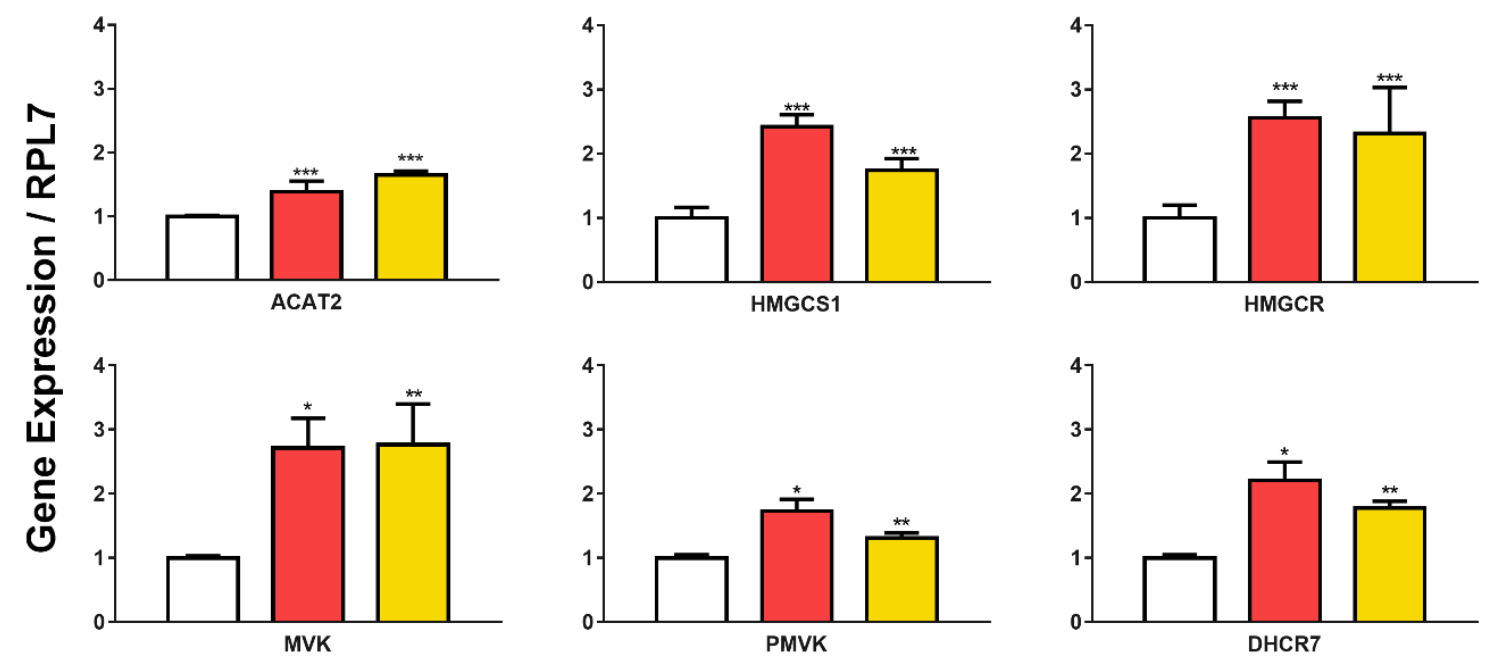

SCRAMBLED $\square$ CTSB KD $\square$ GAA KD

Figure 16. Validation of RNA Seq results in HeLa CTSB and GAA KDs. Expression levels of the principal enzymes of the cholesterol biosynthesis pathway. The transcript levels were determined by the $\Delta \Delta C T$ method using RPL7 (Ribosomal Protein L7) as a reference gene; the plotted value corresponds to the relative expression of the gene (fold change). Samples were centered to one by normalization of the gene expression of the experimental sample to its corresponding control, in this case, scrambled. Graphs show mean \pm SD; $p<0.05(*), p<0.01(* *), p<$ $0.001(* * *)$; Student's t-test; SD: standard deviation. $\mathrm{n}=4$.

Similarly, the qPCR transcript level measurement of the cholesterol biosynthesis pathway in CTSB and GAA KDs were consistent with NGS data of the models of lysosomal malfunction. The activity of the cholesterol biosynthesis pathway was affected in this case (Fig. 16) showing an average of 2 times fold increase in the expression levels of ACAT2 and PMVK; and an increase of between 2 to 3 times fold in HMGCS1, HMGCR, MVK, and DHCR7. Altogether, points towards upregulation of the pathway.

Based on the described results, we conclude that the relevant transcript levels are consistent between RNA-Seq and qPCR measurement. Therefore, we next aimed to unravel the mechanistic steps of the observed phenotypes.

\subsection{Regulation of cholesterol pathway by SREBP1}

We next measured the protein levels, one of the main transcription factors for the synthesis of the lipid, sterol regulatory binding protein 1 (SREBP1) associated with the activation of the cholesterol biosynthesis pathway. When the levels of active SREBP1 are increased, SREBP1 acts as a transcription factor that translocates to the nucleus activating the cholesterol biosynthesis pathway (Shao and Espenshade, 2012). 
In a cellular model of mitochondrial dysfunction such as UQCRC1 KD, SREBP1 was inactive, as measured by WB, (Fig. 17A), in agreement with the downregulation of the pathway.

A

SREBP1

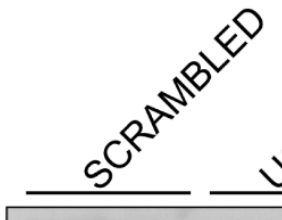

GAPDH

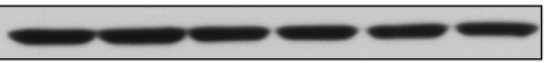

B

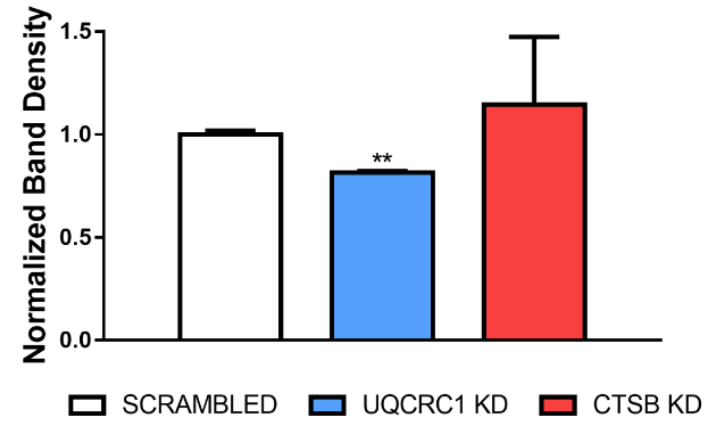

Figure 17. UQCRC1 KD HeLa decreases the protein levels of active SREBP1. A) UQCRC1 KD HeLa showed a significant decrease in the protein levels of SREBP1; in CTSB KD HeLa there was no change observed. B) Quantification of the Western blot. For the quantification, two biological replicates were used. Control (scrambled) value was centered at 1 , and the HeLa KDs values were normalized to the control (scrambled). Graph presents mean \pm SD; $p<0.01(* *)$; Student's t-test; SD: standard deviation.

Simultaneously, we tested if the differential regulation of cholesterol biosynthesis is conserved in other cell lines, considering that HeLa cells are cancer cells and have physiology far from that of healthy cells in tissues (Hyman and Simons, 2011). We, therefore, introduced mouse embryonic fibroblasts (MEFs) since fibroblasts are cells from mesenchymal origin located in several tissues and can respond to multiple stimuli (Qiu et al., 2016).

\subsection{Cholesterol biosynthesis pathway is downregulated in a mouse model of mitochondrial dysfunction}

By employing standard procedures, we have obtained MEFs from the Ndufs $4 \mathrm{KO}$ mouse, a mouse model of mitochondrial malfunction lacking the NDUFS4 subunit of the respiratory chain complex I. Using these MEFs we obtained similar results to those presented for the UQCRC1 KDs in HeLa cells. 
A

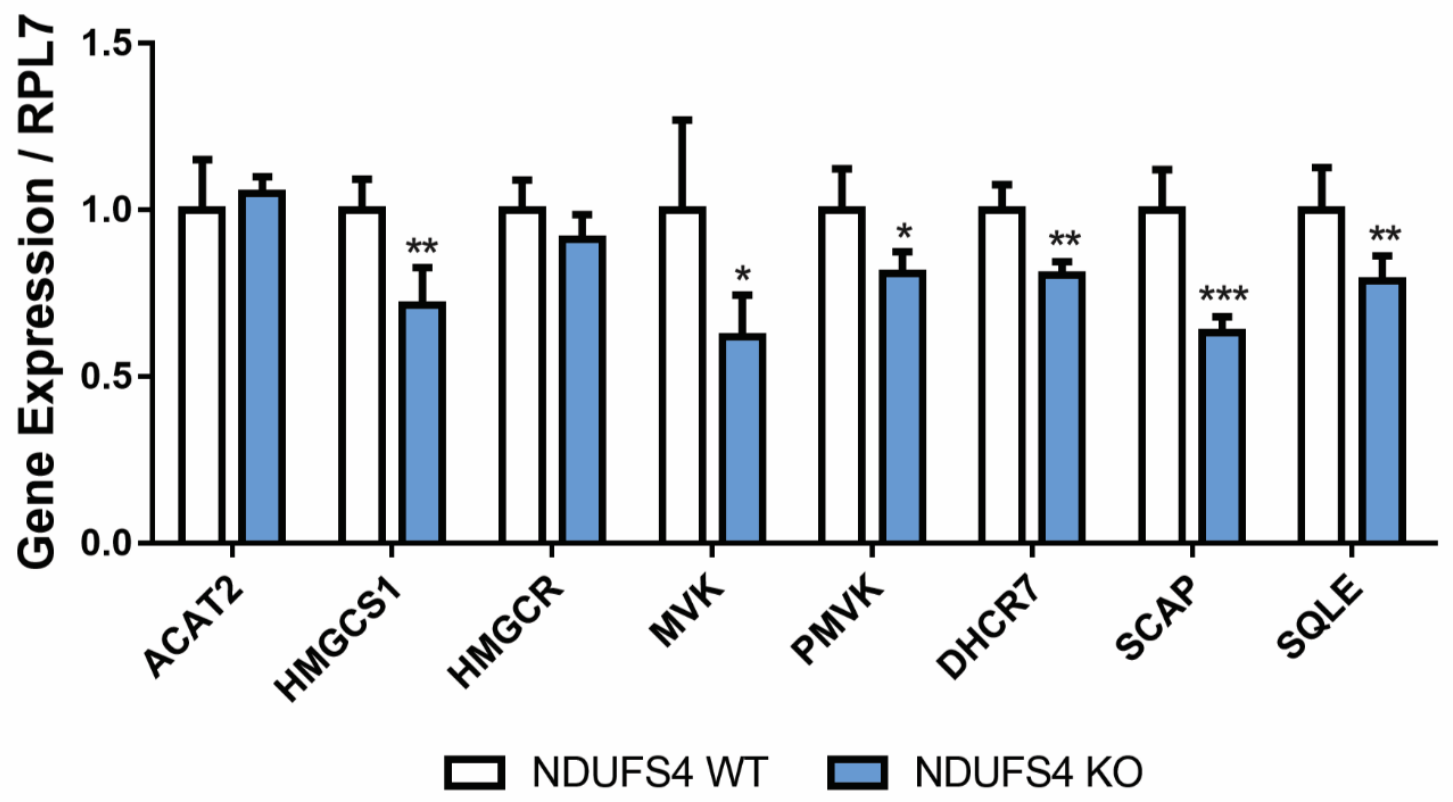

B
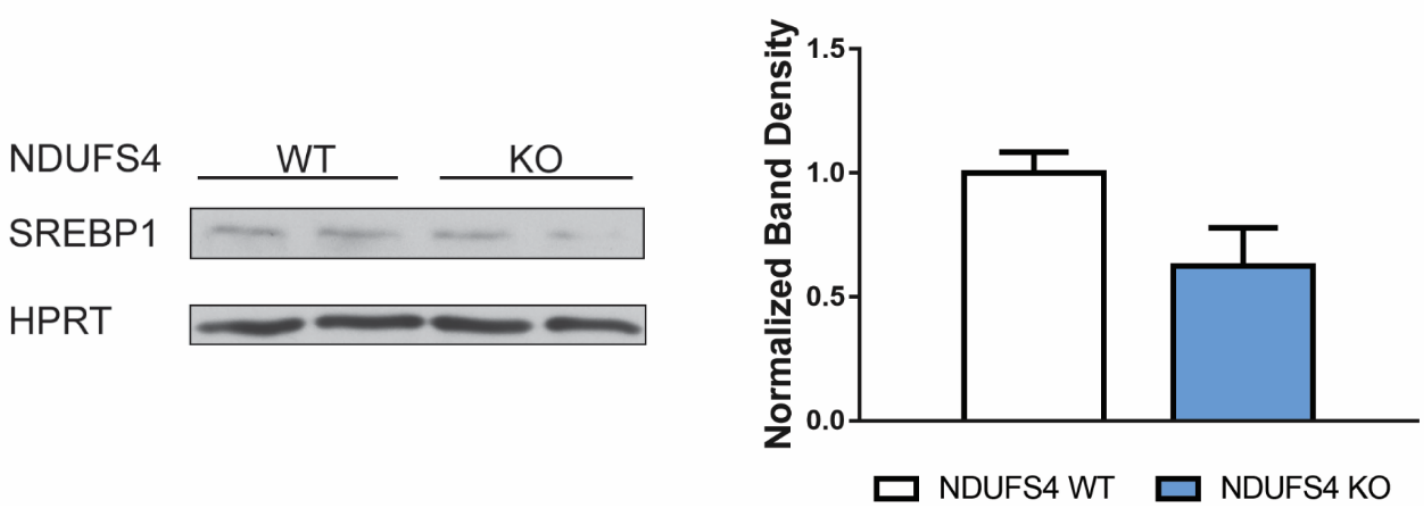

Figure 18. Downregulation of cholesterol synthesis in Ndufs4 KO MEFs. A) Downregulation of the transcript levels of the main enzymes involved in the synthesis of cholesterol in Ndufs 4 KO MEFs. The transcript levels were determined by the $\triangle \triangle C T$ method using RPL7 (Ribosomal Protein L7) as a reference gene; the plotted value corresponds to the relative expression of the gene (fold change). Samples were centered to one by normalization of the gene expression of the experimental sample to its corresponding control, in this case, WT. B) Western blot of SREBP1 and its quantification, showing a decreasing trend in the levels of the protein. For the quantification, two technical replicates were used. WT value was centered in one, and the KO value was normalized to the WT. Graphs show mean \pm SD; $\mathrm{p}<0.05\left({ }^{*}\right), \mathrm{p}<0.01\left({ }^{* *}\right), \mathrm{p}<0.001\left(^{* * *}\right)$; Student's t-test; SD: standard deviation. $\mathrm{n}=5$.

There is a reduction (at least 20\%) in the transcript levels of most of the genes from the cholesterol biosynthesis pathway (Fig. 18A). The levels of active SREBP1 tends to decrease when compared with wild-type cells, indicating that the pathway of biosynthesis of cholesterol displays reduced activity (Fig. 18B). 


\subsection{Cholesterol biosynthesis is upregulated in three independent mouse models of lysosomal dysfunction}

To investigate additional models of lysosomal dysfunction, we used three different kinds of MEFs, each a knock out for lysosomal proteins: two corresponding cysteine cathepsins (CtsB and CtsL) and acid alpha glucosidase (Gaa).

A

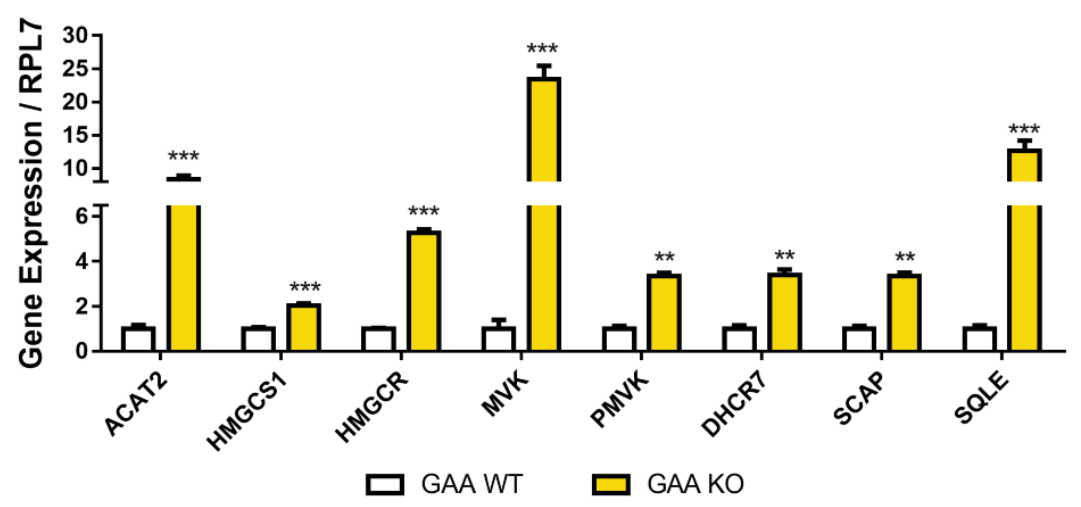

B

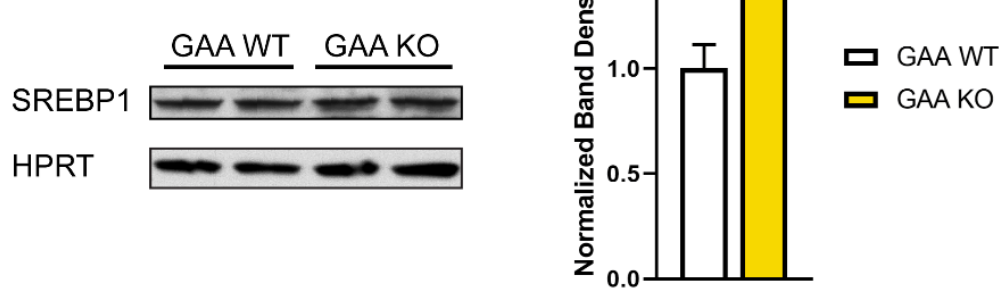

Figure 19. Upregulation of the synthesis of cholesterol Gaa KO MEFs. (A) Upregulation of the levels of the enzymes involved in the cholesterol synthesis pathway in Gaa KO MEFs. The transcript levels were determined by the $\Delta \Delta C T$ method using RPL7 (Ribosomal Protein L7) as a reference gene; the plotted value corresponds to the relative expression of the gene (fold change). Samples were centered to one by normalization of the gene expression of the experimental sample to its corresponding control, in this case, WT (B) Western blot and quantification of SREBP1 active in Gaa KO MEFs, showing a significant increase in the SREBP1 protein levels. For the quantification two technical replicates were used. WT value was centered in one, and the KO value was normalized to the WT. Graphs show mean \pm SEM; $p<0.05\left({ }^{*}\right), p<0.01\left({ }^{* *}\right), p<0.001\left({ }^{* * *}\right)$; Student's t-test; SEM: standard error of the mean. $n=$ 4.

Confirming our predictions, in MEFs, deletion of necessaries enzymes for the normal function of the lysosome such as Gaa, and CtsB and CtsL (Fig. 20) led to an upregulation of genes from the synthesis of cholesterol pathway, suggesting pathway activation. In the case of the Gaa KO, we observed a strong increase in most of the transcript levels of 4 times fold in the genes from the cholesterol biosynthesis pathway (Fig. 19A). Although not as strong as in the Gaa KO MEFs, we also observed a significant increase in the cholesterol biosynthetic pathway in CtsB and CtsL KO (Fig. 20A) even though the increase of the transcript levels was more in CtsB than in CtsL. 
Concerning the levels of active SREBP1, we observed a significant increase in its levels in all lysosomal dysfunction model in MEF, Gaa KO MEFS (Fig. 19B), and CtsB and CtsL KO MEFs (Fig. 20B).

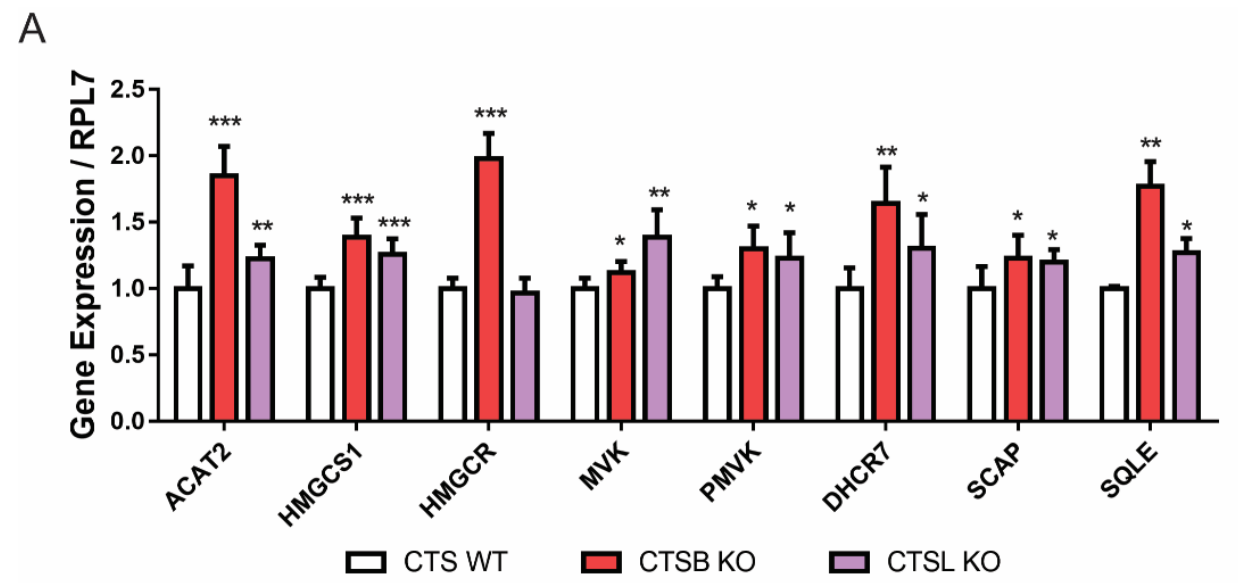

B

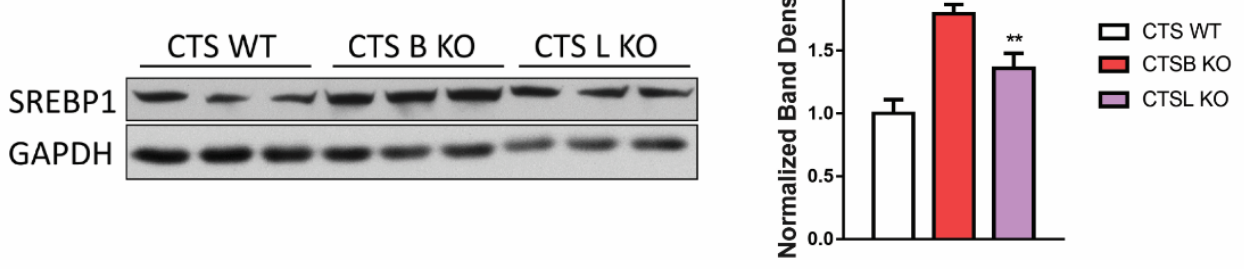

Figure 20. Upregulation of the synthesis of cholesterol in two different models of lysosomal dysfunction. (A) Upregulation of the levels of the enzymes involved in the cholesterol synthesis pathway in CtsB and CtsL KO MEFs. The transcript levels were determined by the $\triangle \triangle C T$ method using RPL7 (Ribosomal Protein L7) as a reference gene; the plotted value corresponds to the relative expression of the gene (fold change). Samples were centered to one by normalization of the gene expression of the experimental sample to its corresponding control, in this case, WT (B) Western blot and quantification of SREBP1 active in CtsB and CtsL MEFs, showing a significant increase in the SREBP1 protein levels. (C) Quantification of western blot. For the quantification, three technical replicates and three biological replicates were used. WT value was centered in one, and the KO value was normalized to the WT. Graphs show mean \pm SD; $p<0.05\left({ }^{*}\right), p<0.01(* *), p<0.001\left({ }^{* *}\right)$; Student's t-test; SD: standard deviation. $n=5$.

Taken together, all results obtained from the different MEFs KO are comparable to the obtained from the HeLa KDs cells. Mitochondrial dysfunction cells repress the cholesterol synthesis pathway, and lysosomal dysfunction activates the pathway.

\subsection{Cholesterol biosynthesis activity is downregulated in Ndufs4 $\mathrm{KO}$ mice and upregulated in Gaa KO mice}

To further analyze if this pattern was reproducible in vivo, not only in HeLa or MEF. We analyzed the expression data of the main enzymes involved in the cholesterol biosynthesis pathway in liver samples of Ndufs $4 \mathrm{KO}$ and Gaa KO. 
A

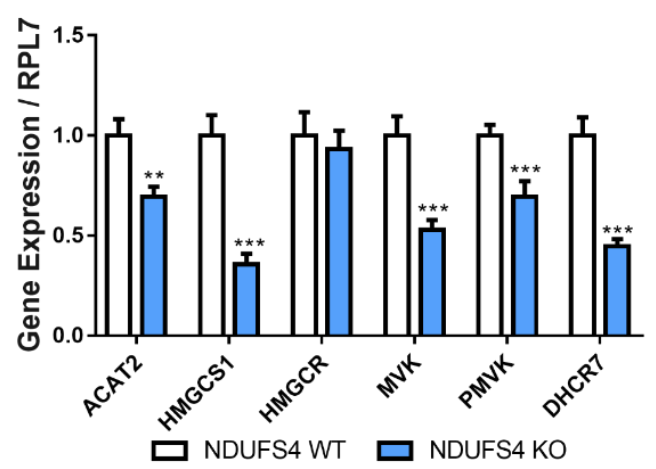

B

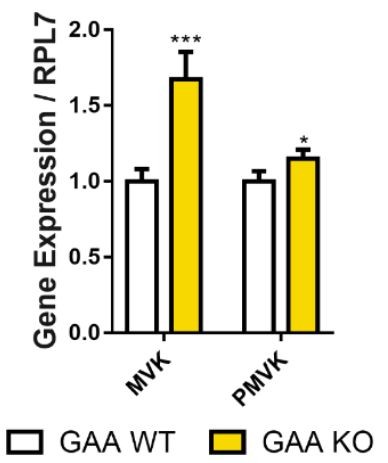

Figure 21. Transcript levels of tissue samples. Transcript levels of enzymes involved in the cholesterol biosynthesis pathway in 6 weeks Ndufs4 KO mice (A) and six months Gaa KO mice (B). The model of mitochondrial dysfunction (A) shows a decrease in the expression levels, and the opposite result was obtained in the lysosomal model (B). The transcript levels were determined by the $\triangle \Delta C T$ method using RPL7 (Ribosomal Protein L7) as a reference gene; the plotted value corresponds to the relative expression of the gene (fold change). Samples were centered to one by normalization of the gene expression of the experimental sample to its corresponding control, in this case, Ndufs 4 WT or Gaa WT respectively. Graphs present mean \pm SEM; $p<0.05\left({ }^{*}\right), p<0.01\left({ }^{* *}\right), p<0.001\left({ }^{* *}\right)$; Student's t-test; SEM: standard error of the mean. $n=8$.

The obtained results were comparable to the data from the different MEF cells, thus confirming that the regulation of the cholesterol biosynthesis could be affected by organelle dysfunction in liver cells. Lysosomal dysfunction triggers a small increase of $10 \%$ of transcript levels of MVK and PMVK that encode for enzymes of the cholesterol biosynthesis pathway (Fig. 21B). On the contrary, the model of mitochondrial dysfunction displays a decrease between $30-50 \%$ in transcript levels of the cholesterol biosynthesis pathway enzymes, but no of HMGCR (Fig. 21A).

Once our models were validated not only in HeLa but also in MEFs and murine liver samples, we proceeded to identify the main mechanistic steps involved in this process. For this reason, we induced mitochondrial dysfunction in the lysosomal dysfunction models and vice versa. We achieved these aims using a pharmacological approach (detailed below).

\subsection{Pharmacological induction of mitochondrial or lysosome dysfunction}

\subsubsection{Mitochondrial respiratory chain inhibition does not affect the enhanced activity of cholesterol synthesis in lysosomal defects}

Using rotenone (an inhibitor of the mitochondrial complex I), we have impaired the mitochondrial function in CTSB and GAA KD HeLa cells. This treatment resembles the Ndufs4 KO. We analyzed the expression of genes involved in the cholesterol synthesis (Fig. 22) to determine the effect of this treatment. 
SCRAMBLED

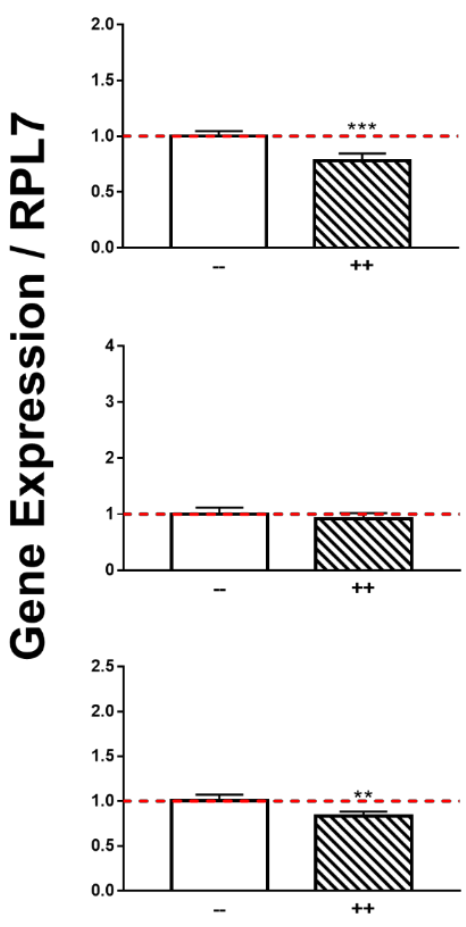

CTSB KD
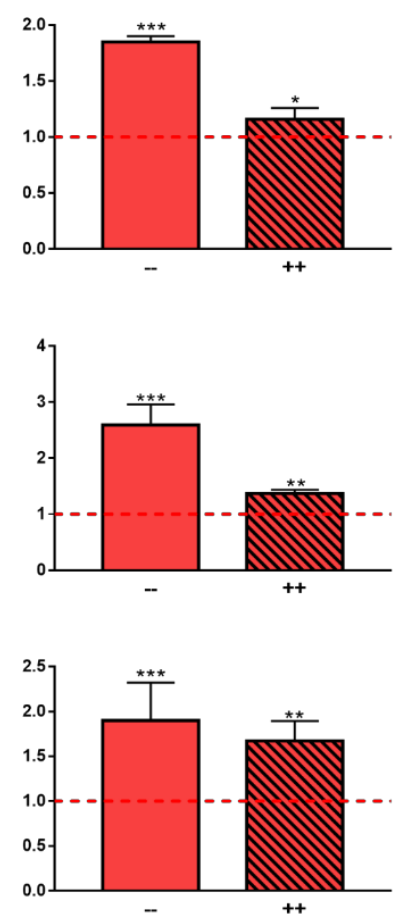

GAA KD
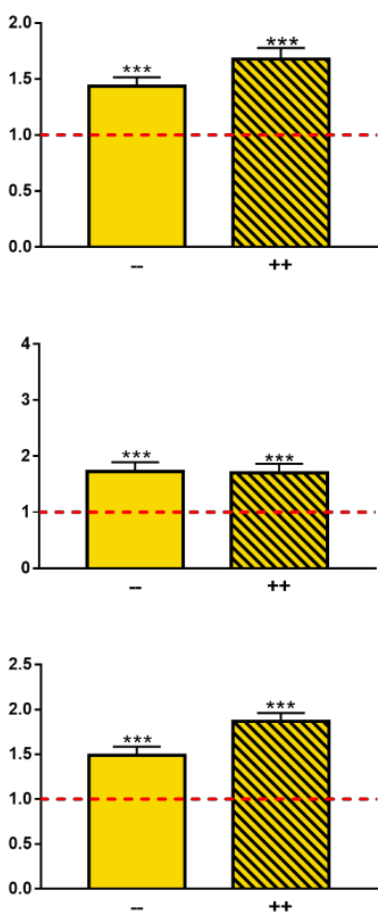

HMGCS1

ACAT2

HMGCR

Figure 22. Lysosomal dysfunction model cells treated with rotenone. Cells were treated with $250 \mu \mathrm{M}$ rotenone during $24 \mathrm{~h}(++)$. Measurement of transcript levels of ACAT2, HMGCS1 and HMGCR in scrambled, CTSB KD and GAA KD. Lysosomal KDs showed a significant increase in the transcript levels even when they are treated with rotenone. The transcript levels were determined by the $\triangle \Delta C T$ method using RPL7 (Ribosomal Protein L7) as a reference gene; the plotted value corresponds to the relative expression of the gene (fold change). Samples were centered to one by normalization of the gene expression of the experimental sample to its corresponding control; in this case, scrambled (-). Graph shows mean \pm SD; $p<0.05(*), p<0.01\left({ }^{*}\right), p<0.001\left(^{* *}\right)$; Student's t-test; SD: standard deviation. $n=4$.

The treatment with rotenone had a different effect in each cell line. In the control cells (with scrambled shRNA applied), the monitored transcript levels were decreased. However, the effect was not consistent in the cellular models of lysosomal dysfunction were not affected by the treatment with rotenone. Only in CTSB KD HeLa, there was a trend to decrease the monitored transcript levels upon rotenone exposure.

To clarify these results, we decided to perform a similar experiment, whereby the trafficking of cholesterol from the lysosome towards the ER would be inhibited in cells with mitochondrial dysfunction. Presumably, such experimental design may help us to elucidate which organellar dysfunction was predominant, the mitochondrial or lysosomal dysfunction.

\subsubsection{Treatment with U18666A increases the transcript levels of main cholesterol biosynthesis enzymes in Ndufs4 KO MEFs}


We measured the transcript levels of genes encoding for enzymes involved in the cholesterol pathway, in non-transfected HeLa cells (Fig. 23A), as well as treated with U18666A (a drug that blocks the transport of cholesterol from the lysosome to the ER). Transcripts were increased more than 5-fold in all of the genes analyzed except in phosphomevalonate kinase (PMVK).

A

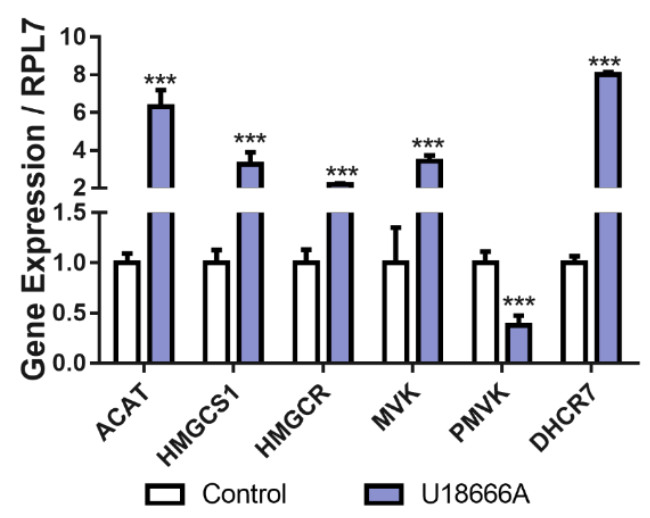

B

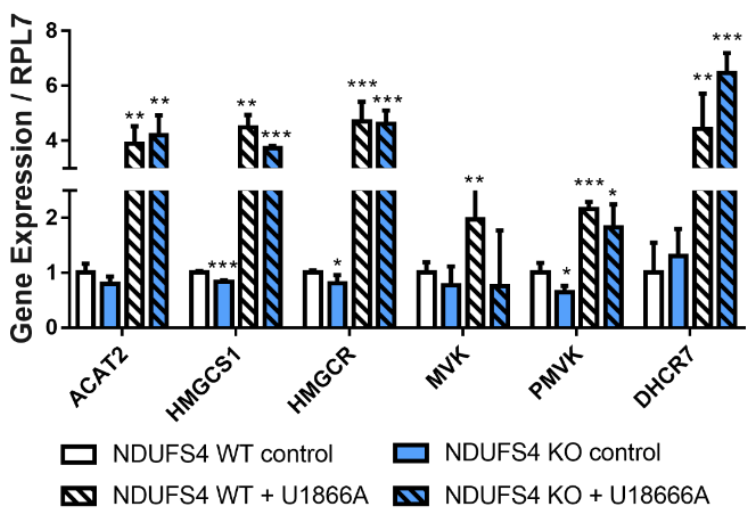

Figure 23. Pharmacological induction of cholesterol biosynthesis pathway. Measurement of transcript levels of main enzymes involved in the cholesterol biosynthesis. (A) HeLa cells treated during $72 \mathrm{~h}$ and (B) Ndufs4 KO MEFs treated during $48 \mathrm{~h}$. Both graphs show a clear upregulation of the transcript levels. The transcript levels were determined by the $\triangle \triangle C T$ method using RPL7 (Ribosomal Protein L7) as a reference gene; the plotted value corresponds to the relative expression of the gene (fold change). Samples were centered to one by normalization of the gene expression of the experimental sample to its corresponding control, in this case, for (A) control and (B) Ndufs4 control. Graphs present mean \pm SD; $p<0.05\left({ }^{*}\right), p<0.01\left({ }^{* *}\right), p<0.001\left({ }^{* *}\right)$; Student's t-test; SD: standard deviation. $n=6$.

Transcript levels of our genes of interest were increased by the treatment in Ndufs4 KO MEFs (Fig. 23B). Here, we expected to observe a reduction in these transcript levels due to the presence of mitochondrial dysfunction in this cell line. However, the phenotype of the mutation was shielded by the effect of the drug.

The amount of cholesterol in the cell is determined in the ER: when the cholesterol content decreases under $5 \%$ of total cholesterol content in the ER membrane, the lipid synthesis is activated (Goldstein et al., 2006). Because of this, our next step was to measure the total content of cholesterol in the cells using different methods.

\subsection{Cholesterol: measurement and reduction}

\subsubsection{Measurement of free cholesterol content in HeLa model cells and CTS KO MEFs by filipin}

We measured unesterified (free) cellular cholesterol content using filipin. This dye has been used for a long time for the measurement of cholesterol in cells, thus helping also in the diagnosis of 
Niemann-Pick disease type C (NPC), a disease caused by defects in cholesterol efflux from lysosomes due to mutations of genes coding for NPC1 and NPC2 proteins (Tängemo et al., 2011).

A

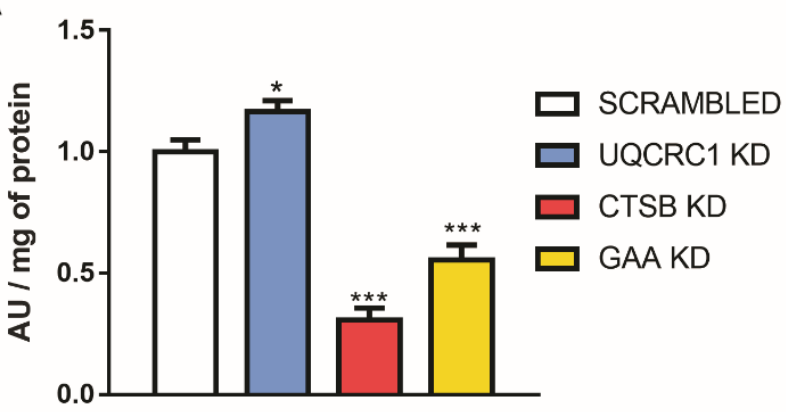

B

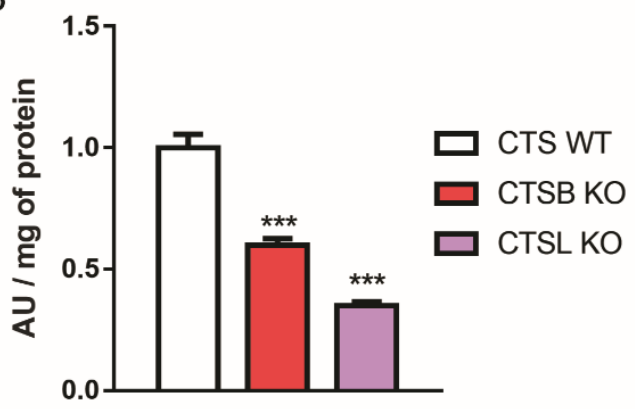

Figure 24. Measurement of free cholesterol content in HeLa KDs and CtsB and CtsL KO MEFs. A) Increase in free content of cholesterol in the UQCRC1 KD cells and decrease in the lysosomal dysfunction models. B) The decrease in free content of cholesterol in Cts KOs MEFs. In all cases, the free cholesterol content was normalized first to protein levels and then to their respective controls (scrambled in HeLa cells and WT in MEFs cells). Graphs show mean \pm SEM; $\mathrm{p}<0.05\left(^{*}\right), \mathrm{p}<0.001\left(^{* * *}\right)$; Student's t-test; SEM: standard error of the mean. $\mathrm{n}=10$.

In the case of UQCRC1 KD HeLa, free content of cholesterol in the cell was increased (Fig. 24A). On the contrary, CTSB KD and GAA KD HeLa present a significant decrease in the free content of cholesterol (Fig. 24A). The same result was observed in Cts KOs MEFs (Fig. 24B). These results may explain the differential response at the transcript level to cholesterol synthesis depending on which organelle is affected.

However, since filipin is rapidly photobleached by UV light and can bind other lipids (Maxfileld and Wüstner, 2012), we decided to use an additional method for total cholesterol detection, implementing the extraction of lipids before the measurement.

\subsubsection{Total cholesterol content is reduced in Ndufs4 KO MEFs and not affected in lysosomal dysfunction}

Here, we employ a more specific method designed for cholesterol detection. The method is enzymatic, and it is based on the oxidation of cholesterol. The $\mathrm{H}_{2} \mathrm{O}_{2}$ produced in the reaction reacts with amplex red producing resorufin. Resorufin is highly fluorescent probe easily detectable (Amundson and Zhou, 1999) (more details in Methods). 
A

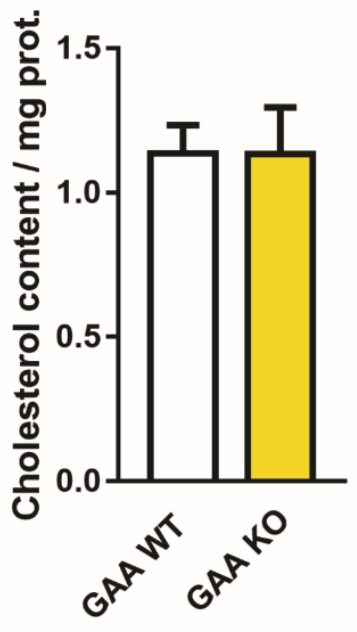

B

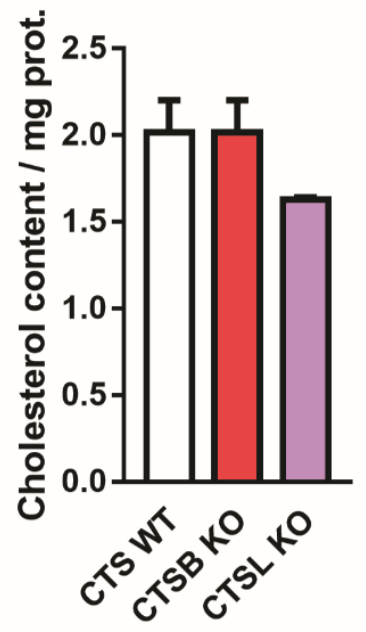

C

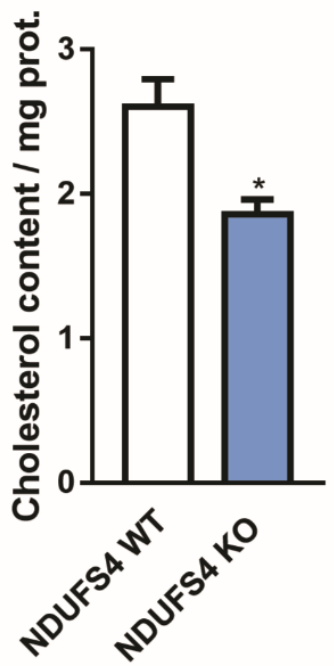

Figure 25. Measurement of cholesterol content by Amplex Red. Graphs displayin cholesterol content normalized to protein amount; in models of lysosomal dysfunction ( $A$ and $B$ ) and a model of mitochondrial dysfunction (C). $A$ and B) Cholesterol content in CtsB, CtsL, and Gaa KO MEFs is not changed. C) Cholesterol content is reduced in Ndufs4 KO MEFs. Quantification indicates mean \pm SEM; $p<0.05\left(^{*}\right)$; Student's t-test; SEM = standard error of the mean; $n=4$.

Using this method, the cholesterol content of the cells was not affected in the case of CtsB, CtsL, and Gaa KO MEFs, although different results were obtained previously with the filipin staining. The Ndufs4 KO MEFs showed a decrease in the cholesterol content compared to the WT (Fig. 25C).

Based on the presented results, we consider the possibility that, in our model cell lines, the total cholesterol content remains unchanged, however, that its content in the different organelles is affected. Therefore, we further measured the cholesterol content in mitochondria.

\subsubsection{Mitochondrial cholesterol is increased in Ndufs4 KO MEFs}

In order to tackle the question defined above, we first extracted the mitochondria enriched fraction form Ndufs4 and Gaa KO MEFs. Next, we performed the measurement of cholesterol contends using the amplex red method. 
A

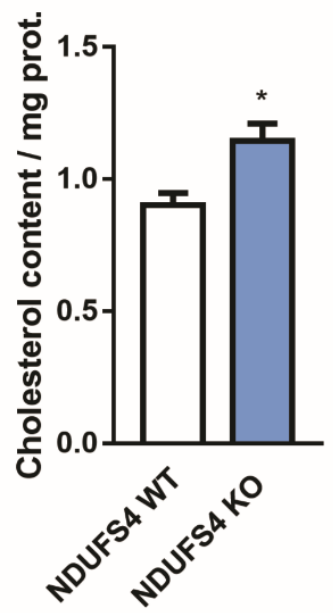

B

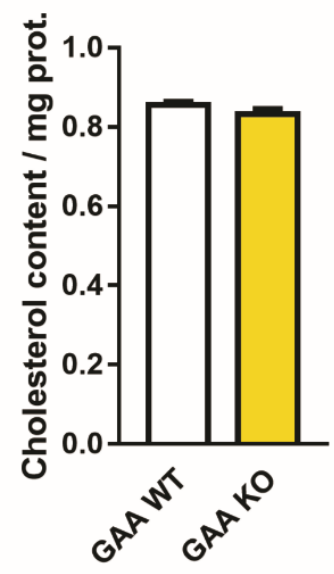

Figure 26. Measurement of mitochondrial cholesterol content by Amplex Red. Mitochondria were extracted from cells obtained from a cell extract fraction enriched in mitochondria. From this fraction, the cholesterol content was measured, and the protein concentration was determined. The cholesterol content of the mitochondrial fraction was significantly increased in Ndufs4 KO MEFs(A) and not changed in Gaa KO MEFs(B). Cholesterol content was normalized to the protein concentration of each sample. Quantification shows mean \pm SEM; Student's t-test; $p<0.05$ $(*) ;$ SEM = standard error of the mean; $n=3$

Cholesterol content of the enriched mitochondrial fraction in Ndufs 4 KO cells (Fig. 26A) displayed a significant increase. The opposite result was observed in total cholesterol content. However, there was no change in cholesterol content in Gaa KO mitochondria (Fig. 26B).

For the next set of experiments, we aimed to remove or add cholesterol to the cells. One of the methods used to remove cholesterol is the treatment with cyclodextrin, the compound with the ability to bind cholesterol. Moreover, because cholesterol is not soluble in water, one of the most used forms of cholesterol delivery to the cells is using cyclodextrins as carriers.

\subsection{Treatment with hydroxypropyl- $\beta$-cyclodextrin (HPCD) upregulates the cholesterol biosynthesis in CtsB and $L$ KO}

To achieve the aim of depleting cholesterol from the cells, we used a cyclodextrin to subtract the cholesterol from the cells, thus lowering the overall cellular cholesterol content. In this case, we use hydroxypropyl- $\beta$-cyclodextrin (HPCD) (Infante and Radhakrishnan, 2017). 


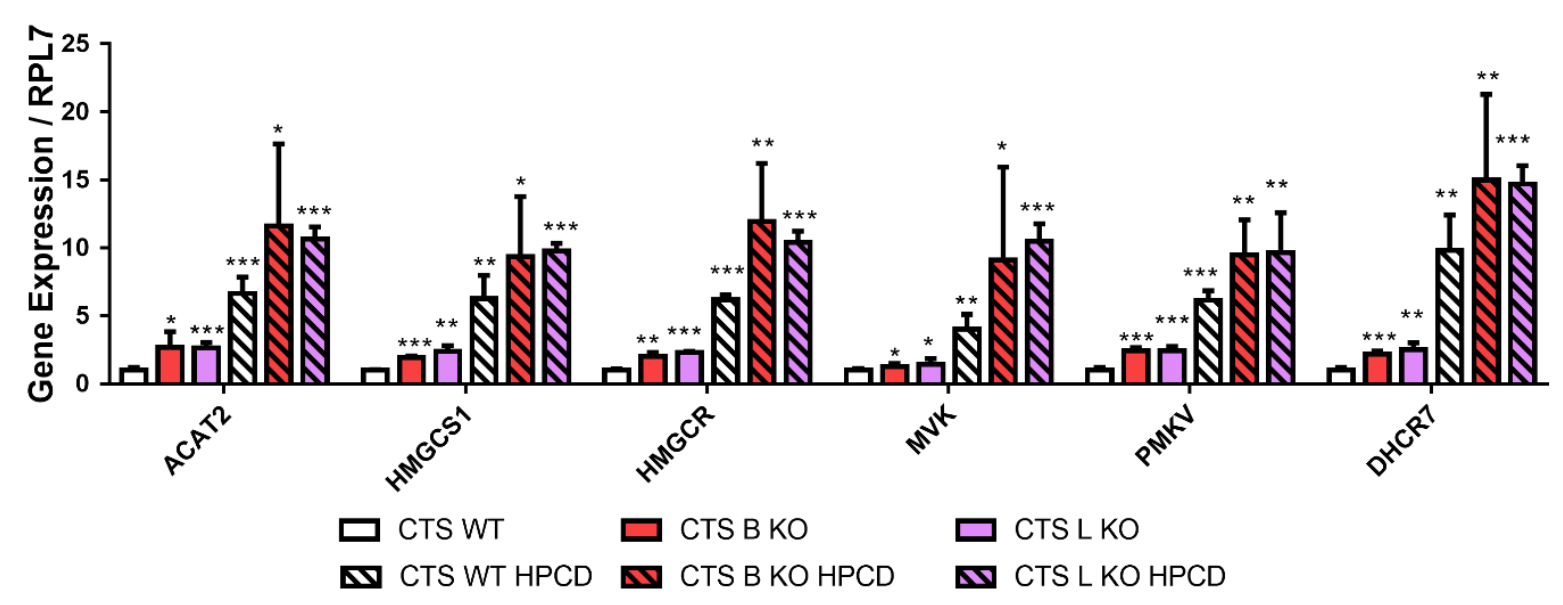

Figure 27. Analysis of the transcript levels of cholesterol-related genes in CtsB and CtsL KO MEFs treated with HPCD. Cells were treated with $1 \% \mathrm{w} / \mathrm{v}$ HPCD during $4 \mathrm{~h}$. CtsB and CtsL KO MEFs showed an upregulation of the transcript level in the presence of HPDC. The transcript levels were determined by the $\triangle \triangle C T$ method using RPL7 (Ribosomal Protein L7) as a reference gene; the plotted value corresponds to the relative expression of the gene (fold change). Samples were centered to one by normalization of the gene expression of the experimental sample to its corresponding control, in this case, CTS WT. Graph shows mean \pm SD; $p<0.05\left({ }^{*}\right), p<0.01(* *), p<0.001\left(^{* * *}\right)$; Student's t-test; SD: standard deviation. $n=6$.

The treatment with HPCD led to increased transcript levels of all the enzymes involved in the cholesterol synthesis in CtsB and CtsL KO MEFs (Fig. 27).

\subsection{Treatment with HPCD in Ndufs4 KO MEFs increased the transcript levels of genes from cholesterol biosynthesis}

Our models of mitochondrial dysfunction downregulate the expression of the enzymes involved in cholesterol synthesis. On the contrary, the addition of HPCD to the medium upregulates the cholesterol synthesis. The treatment with HPCD in the Ndufs4 KO MEFs produced a clear upregulation of the transcript levels (Fig. 17). The effect of the mitochondrial dysfunction in the cholesterol biosynthesis is suppressed by the addition of HPCD.

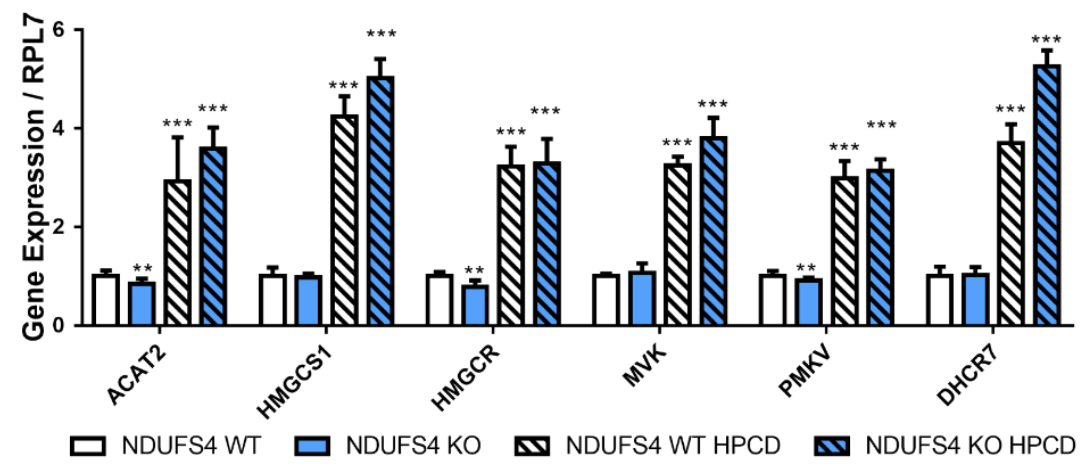

Figure 28. Analysis of the transcript levels of cholesterol-related genes in Ndufs4 MEFs treated with HPCD. Treatment with $1 \% \mathrm{w} / \mathrm{v}$ HPCD during $4 \mathrm{~h}$. The treatment upregulates the studied genes in Ndufs4 MEFs. The transcript 
levels were determined by the $\Delta \Delta C T$ method using RPL7 (Ribosomal Protein L7) as a reference gene; the plotted value corresponds to the relative expression of the gene (fold change). Samples were centered to one by normalization of the gene expression of the experimental sample to its corresponding control, in this case, Ndufs 4 WT. Graph presents mean \pm SEM; $p<0.01(* *), p<0.001\left({ }^{* *}\right)$; Student's t-test; SEM: standard error of the mean. $n=6$.

In both models, cells with mitochondrial (Fig. 28) and lysosomal dysfunction (Fig. 27), the depletion of cholesterol from the cell triggers upregulation of the cholesterol biosynthesis pathway.

One of our goals was to determinate the possible mechanism involved in our models of organelle dysfunction that modulates the cholesterol biosynthesis. Our hypotheses involve two central kinases that regulate the metabolism in the cell (Inoki et al., 2011): (i) the pathway regulated by mTORC complex is leading to the direct activation of SREBP1 and inhibition of lipin1; or (ii) AMPK regulates the pathway in an inhibitory way by phosphorylating HMGCR and ACC (acetyl CoA carboxylase).

\subsection{Determination of signaling pathways involved}

\subsubsection{Blocking mTORC activity enhances the cholesterol biosynthesis pathway in CtsB KO MEFs}

To address the role of $\mathrm{mTORC}$ inhibition in the regulation of cholesterol biosynthesis, we treated CtsB KO MEFs with $250 \mathrm{~nm}$ Torin-1 (Fig. 18). Torin-1 is a potent inhibitor of mTORC1 and mTORC2, which in turn regulates the expression of SREBPs. We measured the transcript levels of genes encoding for the enzymes of the cholesterol biosynthesis pathway.

These cells showed no change in the basal activity of mTORC, determined by the WB measurement of the phosphorylation level of ribosomal protein S6 (S6), an established target of mTORC1 (Fig. 29A). Under basal conditions, the relevant transcript levels in CtsB KO cells are increased by approximately $20 \%$. However, when CtsB KO cells are treated with Torin-1, the same transcript levels display a 2-4 fold increase (Fig. 29B). This indicates that the effect produced by the treatment with Torin-1 has a stronger effect on cholesterol biosynthesis than the absence of CtsB (Fig. 29B).

Next, we performed the same experiment in the model of mitochondrial dysfunction. 
A

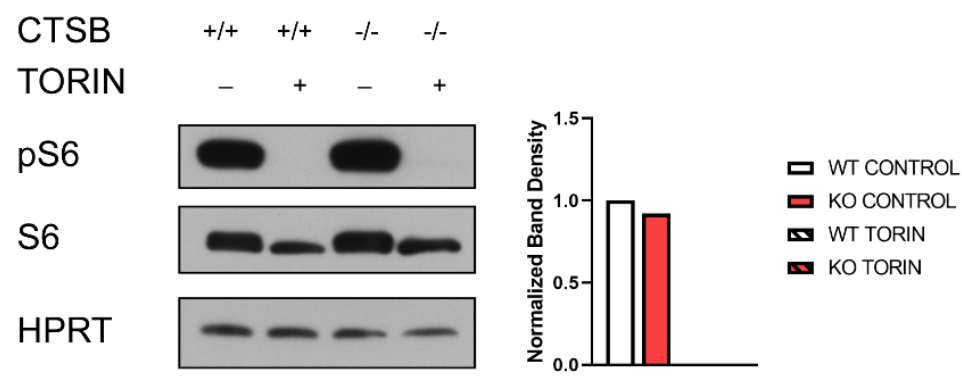

B

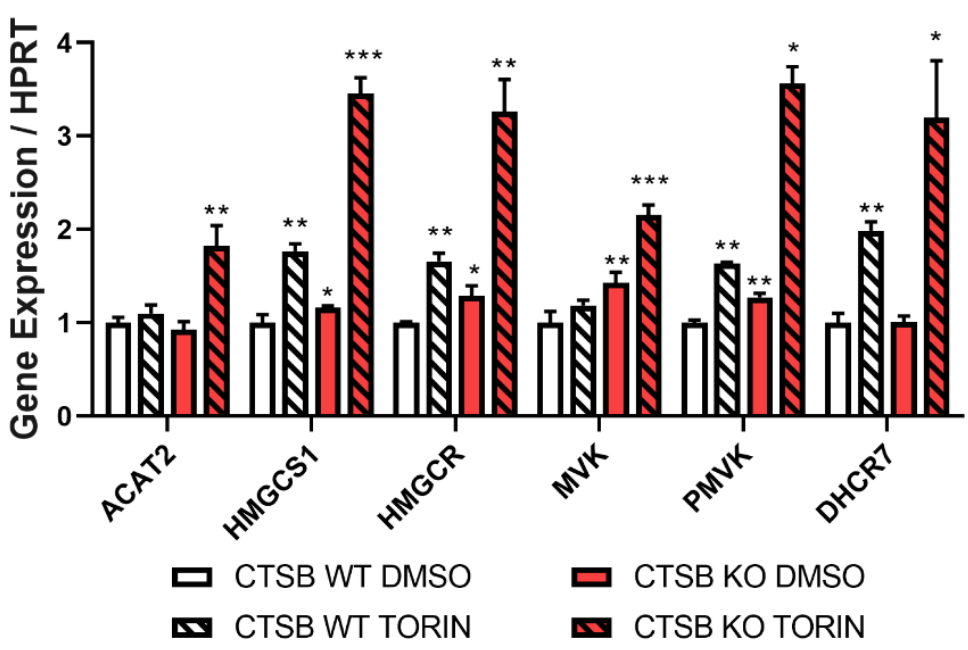

Figure 29. Treatment with Torin increases the cholesterol biosynthesis pathway in CtsB KO MEFs. A) Left) representative western blot of ribosomal protein $\mathrm{S6}$ (S6) and its phosphorylated form. Right) quantification. Samples values were normalized to CTS WT (-) which was centered in one. B) Data showing the upregulation of transcripts of the main genes involved in the cholesterol biosynthesis. The transcript levels were determined by the $\Delta \Delta C T$ method using HPRT (Hypoxanthine Phosphoribosyltransferase 1) as a reference gene; the plotted value corresponds to the relative expression of the gene (fold change). Samples were centered to one by normalization of the gene expression of the experimental sample to its corresponding control, in this case, CTS WT DMSO. Values are normalized to the WT control. Graph shows mean \pm SD; $p<0.05\left({ }^{*}\right), p<0.01\left({ }^{* *}\right) \mathrm{p}<0.001\left({ }^{* *}\right)$; Student's t-test; SD: standard deviation. $n=4$.

\subsubsection{Blocking mTORC activity does not affect the cholesterol biosynthesis pathway in Ndufs4 KO MEFs}

The Torin 1 treatment was also applied to Ndufs4 KO MEFs (250 nM Torin 1 during 4h). In this system, we could not observe a significant change in the expression levels of cholesterol biosynthesis genes upon Torin 1 treatment, compared to the non-treated cells (Fig. 30B). A further change in the relevant transcript levels, detected in KO cells, is absent upon Torin 1 treatment (Fig. 30B). 
Measurement of the MTORC activity via the phosphorylation level of ribosomal protein S6 (S6) (Fig. 30A) revealed an increased mTORC activity in Ndufs4 KO MEFs. Therefore, we could have expected an increase in the cholesterol biosynthesis enzymes transcript levels due to mTORC activation. Because of that, we hypothesize that the repression of the cholesterol synthesis in this model of mitochondrial dysfunction is independent of the regulation of mTORC.

A
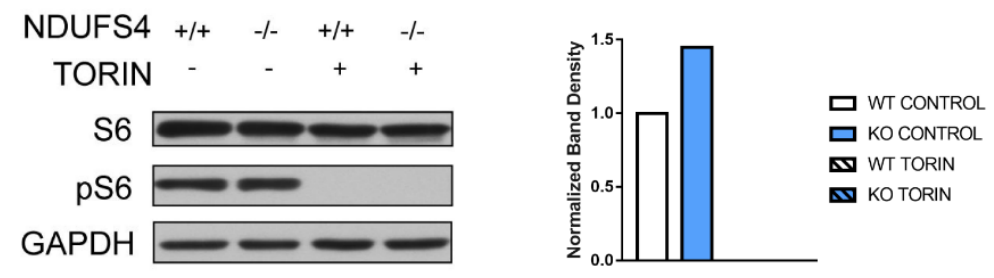

B

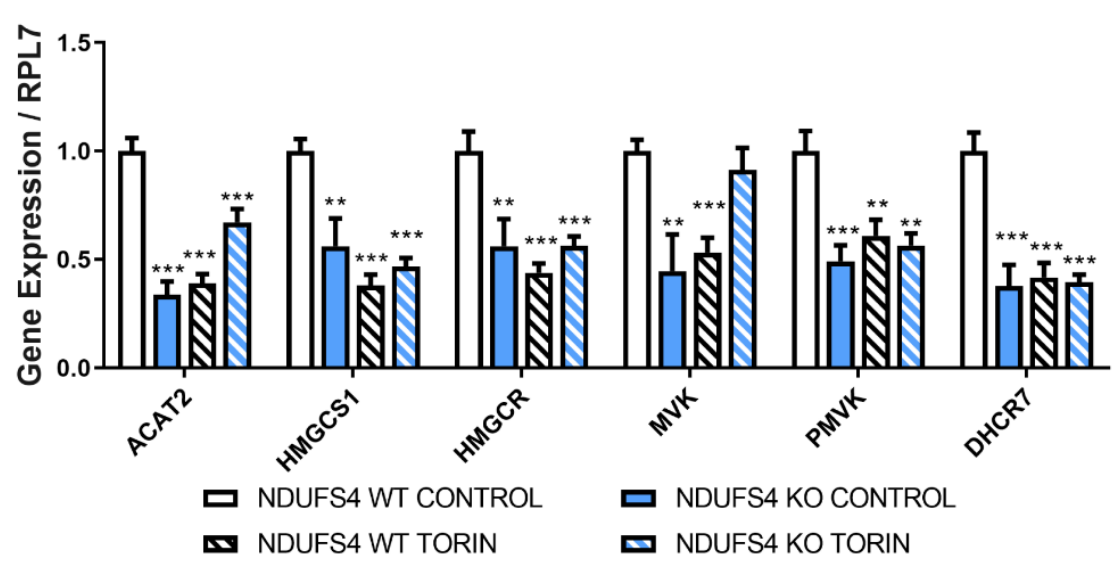

Figure 30. Treatment with Torin does not affect the cholesterol biosynthesis pathway in Ndufs4 KO MEFs. A) Left) representative western blot of ribosomal protein $\mathrm{S6}$ (S6) and its phosphorylated form. Right) quantification of the western blot. Samples values were normalized to NDUFS WT (-) which was centered in one. B) Data showing the downregulation of transcripts of the main genes involved in the cholesterol biosynthesis. The transcript levels were determined by the $\triangle \Delta C T$ method using RPL7 as a reference gene; the plotted value corresponds to the relative expression of the gene (fold change). Samples were centered to one by normalization of the gene expression of the experimental sample to its corresponding control, in this case, NDUFS4 WT CONTROL. Graphs present mean \pm SD; $p$ $<0.05\left(^{*}\right), \mathrm{p}<0.001\left(^{* *}\right) \mathrm{p}<0.001\left({ }^{* *}\right)$; Student's t-test; SD: standard deviation. $\mathrm{n}=6$.

The effect of the treatment with Torin 1 varied in each model cell line: CtsB KO cells showed an increase in the transcript levels of interest, but Ndufs $4 \mathrm{KO}$ cells were not affected even despite the increased activity of mTORC. Taken together, we propose that the mTORC pathway is an unlikely main contributor to this differential regulation of cholesterol biosynthesis.

However, another main kinase regulates the cholesterol synthesis, AMP-activated protein kinase (AMPK). Its effect on the metabolic activity of cells can be divided into two main contributions: i) the inhibition of anabolism to minimize ATP consumption (inhibition of lipids synthesis) and ii) the stimulation of catabolism to stimulate ATP production (activating fatty acid oxidation) (Herzig and Shaw, 2018; Kim et al., 2016)(Kim et al., 2016)(Kim et al., 2016)(Kim et al., 2016)(Kim et al., 
2016)(Kim et al., 2016)(Kim et al., 2016)(Kim et al., 2016)(Kim et al., 2016)(Kim et al., 2016)(Kim et al., 2016)(Kim et al., 2016)(Kim et al., 2016)(Kim et al., 2016)(Kim et al., 2016)(Kim et al., 2016).

\subsection{Upregulation of the cholesterol biosynthesis pathway in AMPK DKO MEFs}

To test the effect of mitochondrial or lysosomal dysfunction on the activity of AMPK, first, we obtained MEF cells from mice with knock-outs of both AMPK subunit $\alpha 1$ and AMPK subunit $\alpha 2$ (AMPK DKO). Second, we induced a perturbation in the organelles using different drugs. We treated AMPK DKO cells during $24 \mathrm{~h}$ with rotenone (inhibitor of complex I), U18666A (inhibitor of the cholesterol transport from the lysosome to the rest of the cell) or co-treatment. After the incubation period, the cells were collected for protein and RNA extraction.

We further analyzed transcript levels of three enzymes involved in the cholesterol synthesis (HMGCS1, HMGCR, and MVK) (Fig. 25). It has previously been reported that AMPK represses the cholesterol biosynthesis. As we expected, AMPK DKO cells present an increase in the monitored transcript levels, compared to the WT cells.

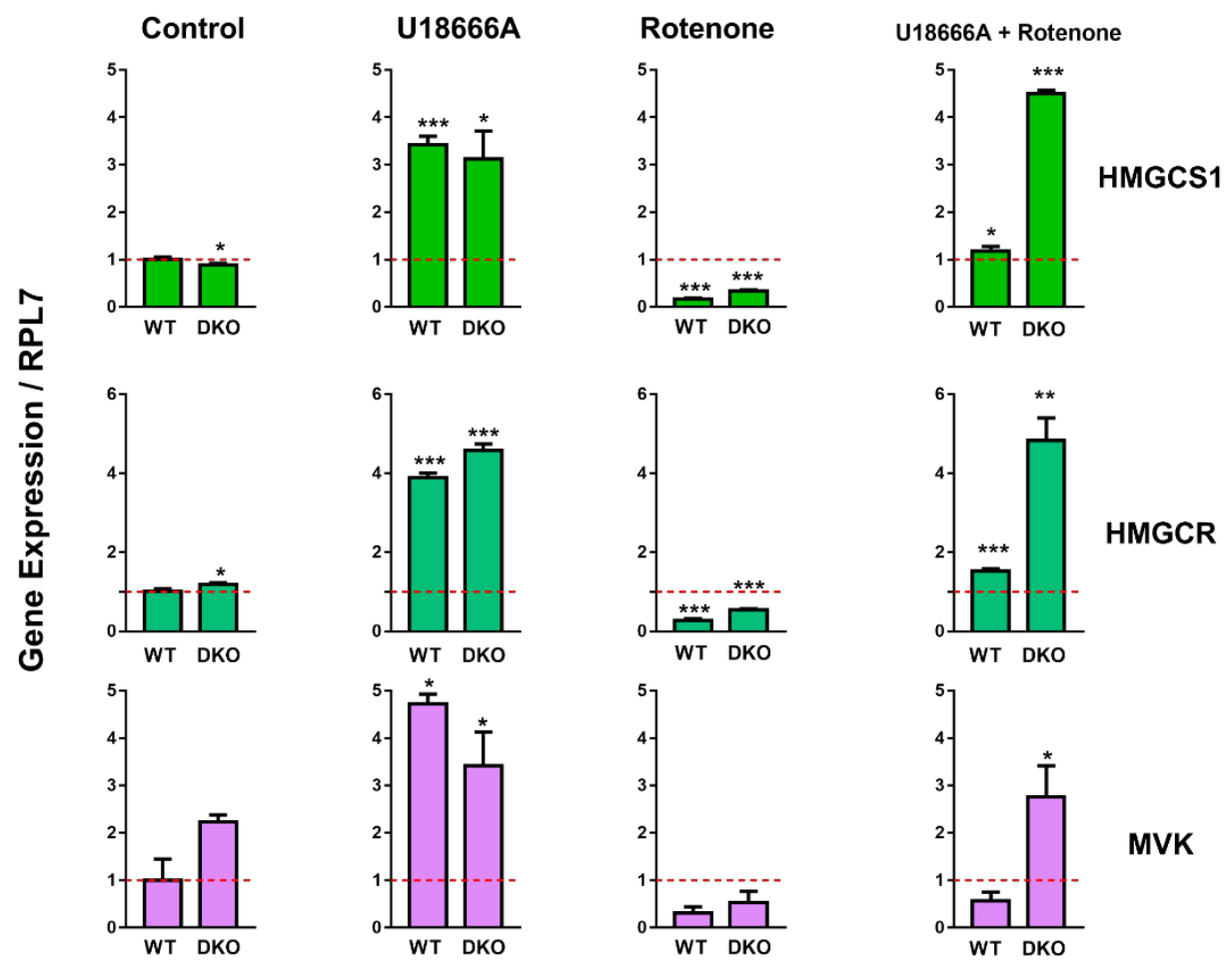

Figure 31. AMPK DKO MEFs cannot restore normal transcript levels of cholesterol biosynthesis genes under

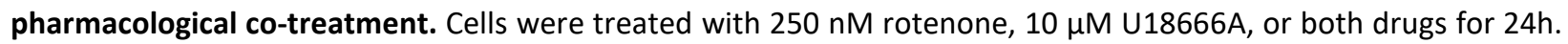
The transcript levels were determined by the $\triangle \triangle C T$ method using RPL7 as a reference gene; the plotted value corresponds to the relative expression of the gene (fold change). Samples were centered to one by normalization of the gene expression of the experimental sample to its corresponding control; in this case, CONTROL WT. Graphs show mean $\pm \mathrm{SD} ; \mathrm{p}<0.05\left({ }^{*}\right), \mathrm{p}<0.01\left({ }^{* *}\right) \mathrm{p}<0.001\left({ }^{* *}\right)$; Student's t-test; SD: standard deviation. $\mathrm{n}=6$. 
The exposure to U18666A generated a significant increase in the monitored transcript levels, in both the WT cells and AMPK DKO. Moreover, cells that underwent rotenone treatment display significantly reduced transcript levels of the HMGCS1 and HMGCR; however, MVK was not affected.

The co-treatment led to an increase in the monitored transcript levels in both cell lines. WT cells treated with U18666A and rotenone showed a 20-50\% increase in the transcript levels compared to WT control cells. However, in AMPK DKO cells, the expression levels of the same transcripts are 4-5 fold increase compared to WT control cells and three times higher than in AMPK DKO control. Based on these results, we showed that following the co-treatment with U18666A and rotenone, AMPK DKO cells are not affected in the same way as WT cells (Fig 31).

Individual treatments with each drug reveal their opposite effects. In WT cells, the treatment with rotenone alone decreased the monitored transcript levels, while the same transcript levels are increased as a result of U18666A treatment.

On the other hand, the treatment with rotenone led to a decrease of the monitored transcript levels by at least $50 \%$ in AMPK DKO MEFs compared with WT control cells (Fig. 31). However, the treatment with U18666A did not additionally increase the transcript levels compared with the WT MEFs under the same conditions.

A

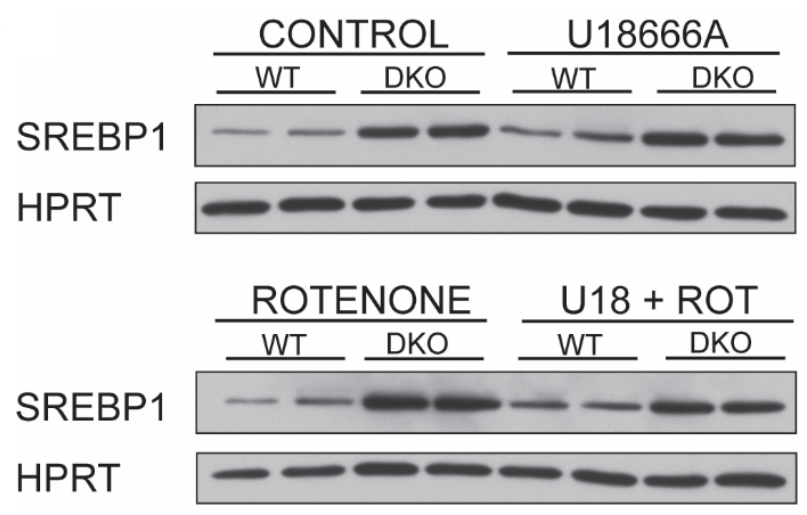

B

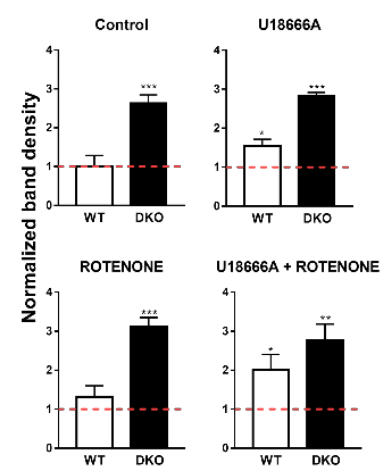

Figure 32. Active SREPB1 protein levels are not affected by pharmacological treatments AMPK DKO MEFs. A) Western blot showing the protein levels of mature SREBP1 under the different treatments, no change was observed in any treatment. The quantification was done using two technical replicates. B) Quantification of the western blot, values were normalized to WT control cells (which was centered in one). Graph presents mean \pm SD; $p<0.05\left({ }^{*}\right), p<$ $0.01\left({ }^{* *}\right) \mathrm{p}<0.001\left({ }^{* *}\right)$; Student's t-test; SD: standard deviation. $\mathrm{n}=2$

Although the expression of the studied genes was affected by the treatments with the applied drugs (Fig. 31), the levels of active SREBP1 were not affected by any treatment (Fig. 32). This 
could be a consequence of inactivation of AMPK, which activates the cholesterol synthesis, thus affecting directly the amount of active SREBP1.

Because of these results, we consider the existence of a mechanism involved in the regulation of cholesterol biosynthesis that is activated when the mitochondria or the lysosome are dysfunctional. This could be a direct regulation (repressing the activity of enzymes involved in the pathway) or indirect (activating another pathway that represses the lipid synthesis) by AMPK. Therefore, we decided to turn to measure the AMPK activity in our lysosomal models.

\subsection{Measurement of AMPK activity in several models of lysosomal dysfunction}

To measure the activity of AMPK in our model cell lines, we quantified the intensity of the band corresponding to AMPK $\alpha$ Thr172 phosphorylation, which is indicative of its activity.

In most of our lysosomal models, the activity of AMPK does not appear to be decreased (Fig. 33). Only Gaa KO MEFs show a significant decrease in AMPK activity. Although not significant, a suggestive trend of decreased AMPK activity is observed in CTSB KD HeLa. 
A

pAMPK

AMPK

HPRT
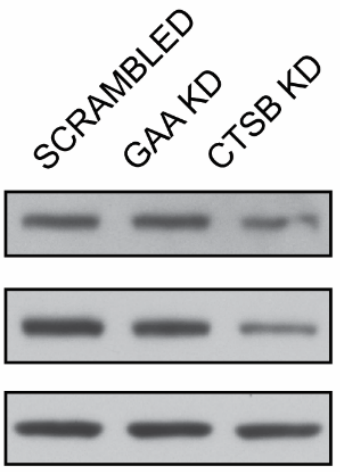

B

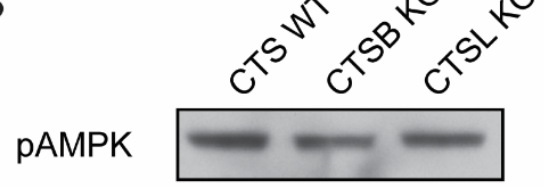

AMPK

HPRT
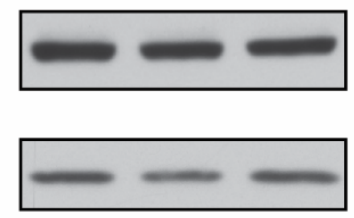

C

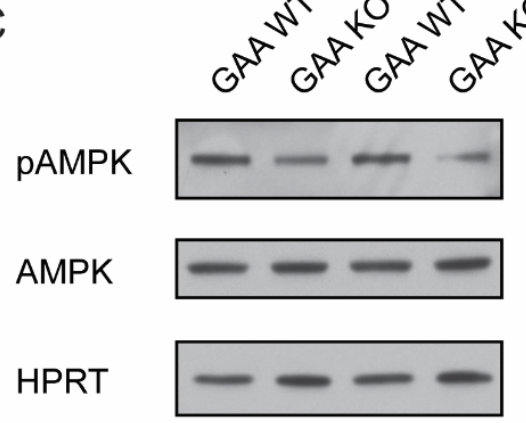

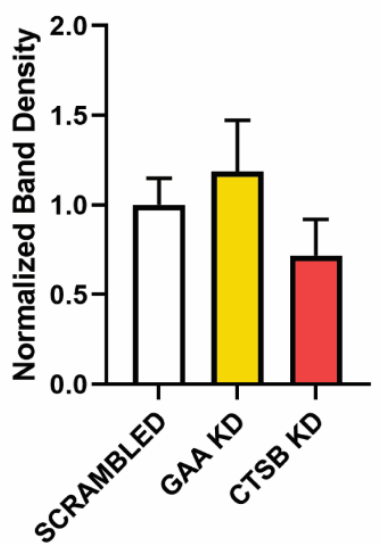
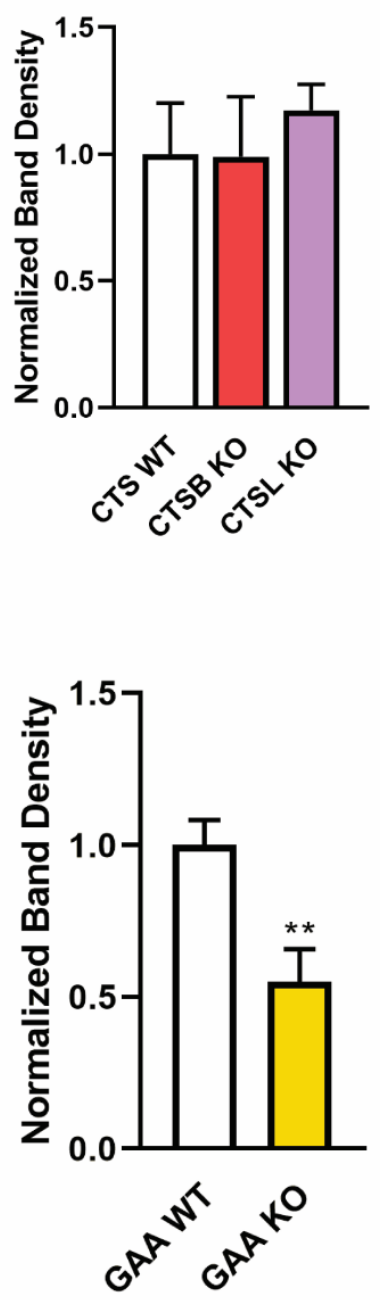

Figure 33. AMPK activity is not decreased in all the studied models of lysosomal dysfunction. Western blot of $A$ ) CTSB and GAA KD HeLa, B) CtsB and CtsL KO MEFs and C) Gaa KO MEFs. Each figure corresponds to western blot (left) and its quantification (right). For the quantification, two technical replicates and two biological replicates were used. WT or scrambled value was centered in one, and the KO or KD value was normalized to the WT. Graph were used presents mean \pm SEM; $\mathrm{p}<0.01(* *)$; Student's t-test; SEM: standard error of the mean. $\mathrm{n}=4$ 
Next, we decided to repeat this experiment with a different model of lysosomal dysfunction. Therefore, we used cells with a knock out of lysosomal membrane glycoprotein 2 (LAMP2). This protein is present in lysosomal membrane and was associated to several functions, such as fusion of lysosomes with phagosomes(Huynh et al., 2007) and has also been proposed to serve as a receptor for chaperone-mediated autophagy (Bandyopadhyay et al., 2008; Cuervo and Dice, 1996).

Recently, LAMP2 has been associated with the binding of lipids in the lysosome, particularly cholesterol (Li and Pfeffer, 2016). Hence, we used Lamp2 KO MEFs as a new model cell line.

\subsection{Characterization of Lamp2 KO MEFs in the context of cholesterol transport}

To further test our hypothesis that cells carrying a lysosomal deficiency display upregulation of the cholesterol biosynthesis pathway, we measured the transcript levels of the main enzymes of the cholesterol biosynthesis pathway in the Lamp2 KO MEFs.

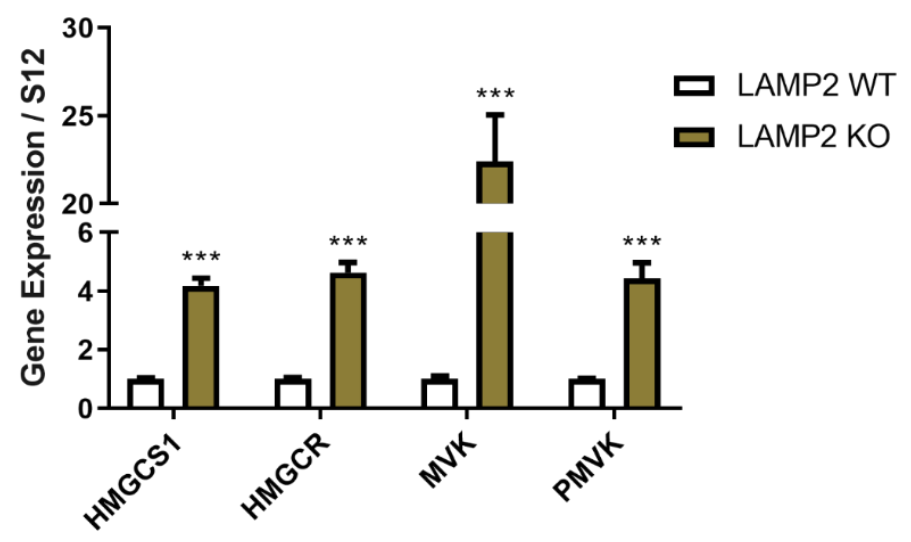

Figure 34. Upregulation of transcript levels involved in the cholesterol biosynthesis pathway in Lamp2 KO MEFs. Increase of the expression levels of the main enzymes involved in the cholesterol biosynthesis pathway. The transcript levels were determined by the $\triangle \Delta C T$ method using RPL7 as a reference gene; the plotted value corresponds to the relative expression of the gene (fold change). Samples were centered to one by normalization of the gene expression of the experimental sample to its corresponding control, in this case, LAMP2 WT. Graph shows mean $\pm S D ; p<0.001\left({ }^{* * *}\right)$; Student's t-test; SD: standard deviation. $\mathrm{n}=5$

The transcript levels of the enzymes of interest displayed a significant increase in Lamp2 KO MEFs (Fig. 34). Next, we evaluated the activity of AMPK by quantification of phosphorylation of AMPK $\alpha$ Thr 172. 

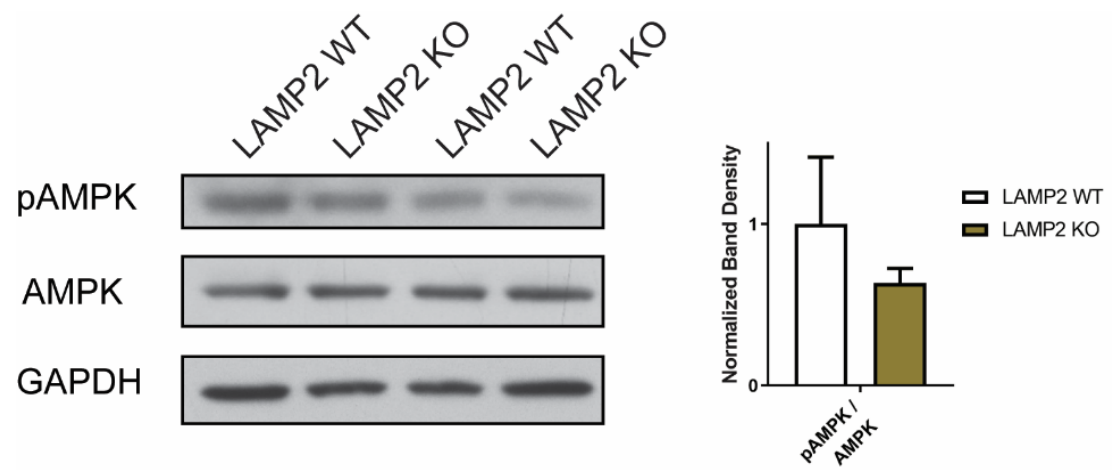

Figure 35. Evaluation of the AMPK activity through the phosphorylation AMPK $\boldsymbol{\alpha}$ Thr 172 . AMPK activity is repressed in Lamp2 KO MEFs. Western blot is showing a trend to decrease (left) and its quantification (right). For the quantification, two technical replicates and two biological replicates were used. WT value was centered in one, and the KO value was normalized to the WT. Graphs show mean \pm SD; SD: standard deviation. $n=4$

AMPK activity tended to decrease (Fig. 35). However, there was no significant change.

One of the essential enzymes in cholesterol synthesis is HMGCR. This enzyme is regulated in several ways, one of them being phosphorylation of Ser 871 (Ser 872 in humans) by AMPK. Once the enzyme is phosphorylated, its activity is repressed.

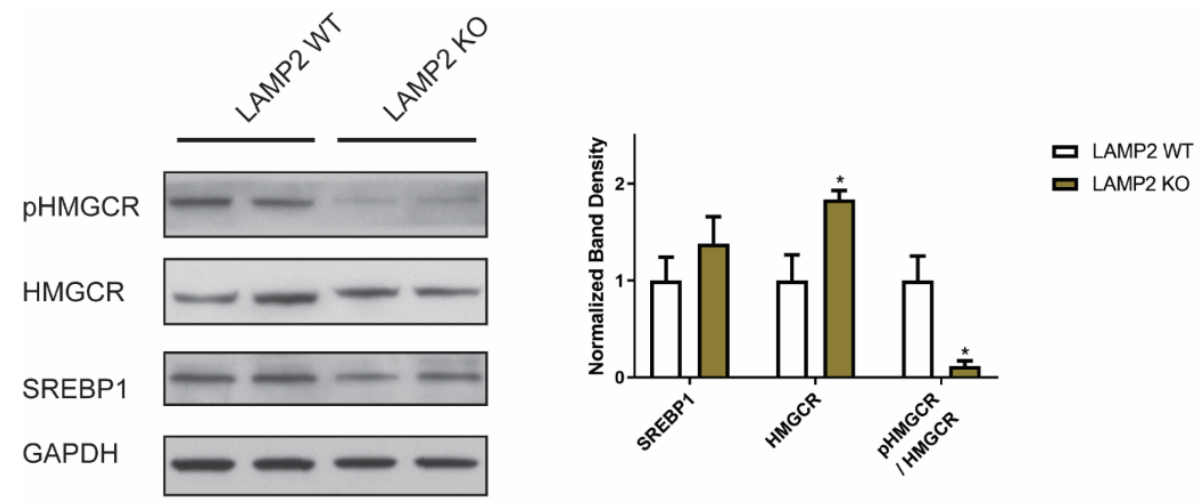

Figure 36. Evaluation of cholesterol pathway activation by SREBP1 and pHMGCR in Lamp2 KO MEFs. The protein levels of SREBP1 were increased, as well as HMGCR. HMGCR was less phosphorylated in Lamp2 KO MEFs than in WT, suggesting activation of cholesterol pathway. For the quantification, two technical replicates and two biological replicates were used. WT value was centered in one, and the KO value was normalized to the WT. Western blot (left) and quantification (right). Graph presents mean \pm SEM; $p<0.05$ (*); Student's t-test; SEM: standard error of the mean. $n=4$.

Analyzing the phosphorylation protein levels of HMGCR, we could observe a decrease. This could be explained due to the decreases in the activity of AMPK. A decrease in the activity correlates with an increase in cholesterol synthesis. Additionally, to support the hypothesis of activation of the cholesterol pathway, the main transcription factor of the cholesterol synthesis, SREBP1, was increased (Fig. 36). 
We further aimed to compare our models of mitochondrial dysfunction (Ndufs4 KO MEFs) with Lamp2 KO MEFs, to investigate if the variation in the AMPK activity regulates the cholesterol synthesis.

\subsection{Comparison of AMPK activity between Ndufs4 KO MEFs and Lamp2 KO MEFs}

Comparing the AMPK activity of Lamp2 KO MEF and Ndufs4 KO MEFs (Fig. 37B), we observed a significant difference. The activity was decreased in Lamp2 KO MEF and increased in Ndufs4 KO MEFs, consistent with our expectations. The levels of active SREBP1 are not changed (Fig. 37D). Regarding HMGCR, Lamp2 KO cells showed a tendency to decrease its levels, but in Ndufs4 KO cells, no changes were observed (Fig. 37C).

From this part of the experiments, we could propose that regulation by AMPK exists in our models through the inhibitory phosphorylation of HMGCR. However, further experiments need to be done in order to clarify this part of the mechanism and also to test it in our initial model of HeLa cells. 
A

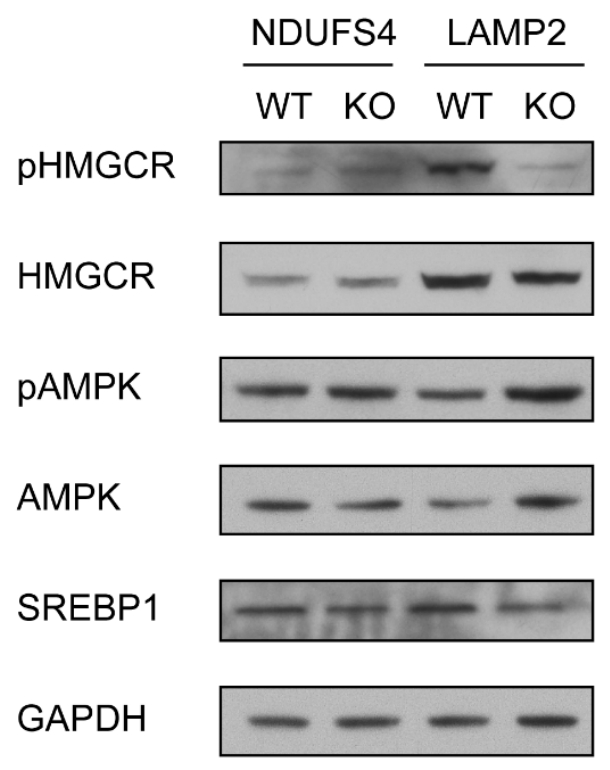

C HMGCR

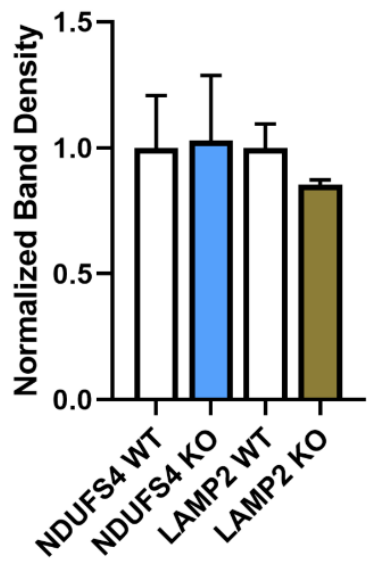

B

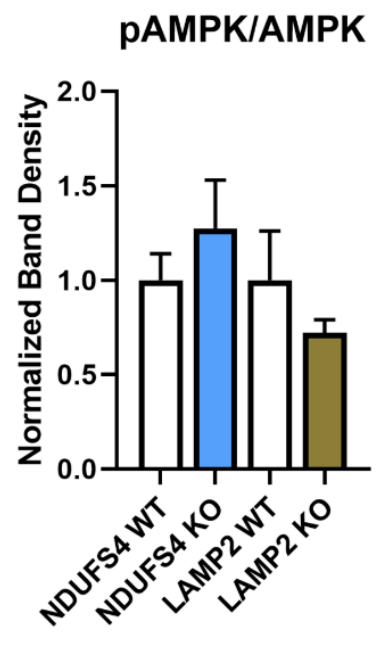

$\mathrm{D}$ SREBP1

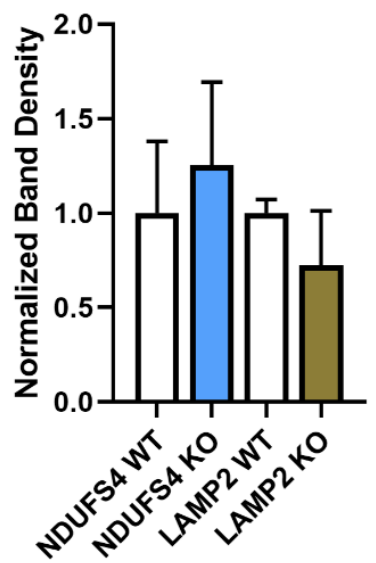

Figure 37. Comparison of Ndufs4 KO and Lamp2 KO MEFs in the activation of the cholesterol biosynthesis pathway. A) Western blot. Quantification of B) AMPK activity. For the quantification were used two technical replicates and three biological replicates; C) total level of HMGCR and D) levels of active SREBP1. For the quantification, two technical replicates and two biological replicates were used. In all the cases, the wild-type values were centered in one, and each KO was normalized to its WT. Graphs show mean \pm SEM; $p<0.05\left({ }^{*}\right)$; Student's t-test; SEM: standard error of the mean. $n=4,{ }^{*} n=6$. 


\section{DISCUSSION}

In this project, we have found that mitochondrial dysfunction and lysosomal dysfunction, both yield consequences on the activity of the cholesterol biosynthesis pathway, albeit of opposite effects. In summary, mitochondrial dysfunction represses the cholesterol biosynthesis pathway while lysosomal dysfunction brought to its enhanced activity. Such differential regulation of the cholesterol biosynthesis pathway is consistent with the phenotypes observed in these models, like a reduction of energy production in mitochondrial dysfunction (Suomalainen and Battersby, 2018), and inability to degrade the macromolecules in lysosomal dysfunction (Parenti et al., 2015). Moreover, our results strongly suggest that AMPK is a likely regulator of the cholesterol biosynthesis pathway. We hypostatize that HMGCR plays a key role in the mechanistic steps linking mitochondria and lysosomes with the cholesterol biosynthesis since it is directly regulated by AMPK via phosphorylation. However, additional mechanistic steps remain to be elucidated, which would allow us for a better understanding of the link between organellar dysfunction and cholesterol availability.

\subsection{Validation of the data obtained from NGS analysis}

\subsubsection{HeLa cells}

We aimed to elucidate the mechanism(s) behind the regulation of cholesterol synthesis in the models of lysosomal and mitochondrial dysfunction (Fig. 12). For this reason, we generated models of lysosomal dysfunction (CTSB and GAA KDs) and mitochondrial dysfunction (NDUFS3 and UQCRC1 KDs). In the case of the mitochondrial dysfunction, we only pursued the experiments with UQCRC1 KD since this model was extensively described by our group, describing the interplay between mitochondria and lysosomes (Fernandez-Mosquera et al., 2019). By taking advantage of available novel technologies, such as next-generation sequencing, which allows us to explore globally the pathways that respond to perturbations of interest, we found the cholesterol biosynthesis pathway to be one of the top affected pathways in the models of mitochondrial and lysosomal dysfunction.

Interestingly, our analyses showed the existence of differential regulation of cholesterol synthesis, which displayed an organelle-specific pattern (Fig. 14). In summary, in the case of mitochondrial dysfunction model, a decline in the synthesis was observed. On the other hand, impaired lysosomal function produced an upregulation in the cholesterol synthesis pathway. Taken together, these results can point to the different role of cholesterol in each of the dysfunction models, i.e., in each of the affected organelles. In the case of mitochondrial dysfunction, where ATP production is reduced (Suomalainen and Battersby, 2018), it is expected 
that most of the cellular anabolic processes (e.g., cholesterol synthesis) would be inhibited or attenuated due to their high energy demand (Boyle, 2005).

The inability of cholesterol to be exported from the lysosome to the ER membrane, like in some lysosomal storages disorder (LSDs), might explain the increase of cholesterol synthesis in the case of lysosomal dysfunction. In this condition, cells would sense a decrease in the cholesterol content in the ER membrane, even though there is plenty of cholesterol in the lysosomes.

We then set out to validate by qPCR the outcomes of the NGS data analyses. At the transcript level, in the HeLa model of mitochondrial dysfunction (UQCRC1 KD), we observed a decrease in the expression of genes of the cholesterol biosynthesis pathway (Fig. 15). This suggests repression in the cholesterol biosynthesis pathway. On the other hand, a different result was reported by (Kühl et al., 2017) where some of the main enzymes involved in the cholesterol biosynthesis, e.g., HMG-CoA synthase (HMGCS1) and farnesyl pyrophosphate synthase (FDPS), had a normal or slightly increased transcript levels when mtDNA gene expression is disrupted. However, the authors are focused on the murine heart tissue, which could be the most likely reason for the discrepancy between the two sets of results.

The canonical pathway of cholesterol biosynthesis activation is regulated by SREBPs. In mammals, two variants of SREBPs are known: SREBP1-a and SREBP1-c. Both variants are produced from the same gene through the use of an alternative transcription start site. The third member of the family is SREBP2, encoded by a separate gene (Brown and Goldstein, 1997). SREBP1-a and SREBP2 are the most predominant isoforms of SREBPs in cultured cell lines; SREBP2 mostly regulates the transcription of the genes involved in the cholesterol metabolism. However, SREBP1a strongly activates global lipid metabolism in rapidly growing cells (Horton et al., 2002). By evaluating the canonical pathway in our model of mitochondrial dysfunction, we found out that SREBP1 was decreased, as expected based on the reduced relevant transcript levels. These results are consistent with the expression data we obtained from the UQCRC1 KDs by qPCR, as wells with the NGS data (Fig. 17).

In some cases, although lysosomes are able to process cholesterol for further use, the inability of lysosomes to degrade some macromolecules, e.g., glycosphingolipids, triggers an accumulation of cholesterol in lysosomes (Walkley and Vanier, 2009). This is consistent with the results obtained from our models of lysosomal storage disorder such as GAA KD and CTSB KD (Fig. 16), which presented an opposite trend compared with the mitochondrial model. In our lysosomal dysfunction models, the results obtained from the qPCR and the activity of SREBP1 point towards the activation of cholesterol biosynthesis pathway (Fig. 17).

Despite the canonical regulator of cholesterol biosynthesis pathway being SREBP2, the consequences of organelle dysfunction are mostly visible at the protein levels of active SREBP1. 
At this point, we cannot discard the possibility that an additional lipid plays an important role in organelle dysfunctions. Because of that, this is an essential issue to elucidate in further studies.

In order to verify that the observed responses to organelle dysfunction were not a cell linespecific response, limited only to HeLa cells, we employed immortalized MEFs with dysfunction either in mitochondria or in lysosomes (Hyman and Simons, 2011).

\subsubsection{Mouse embryonic fibroblasts and mice}

For the model of mitochondrial malfunction, we used MEFs from the Ndufs 4 (core subunit of complex I) KO mice. The mutations in Ndufs 4 subunit and its subsequent dysfunction are studied as a model of Leigh syndrome (Ortigoza-Escobar et al., 2016). The syndrome is a progressive neurodegenerative condition with highly variable symptoms, usually including hypotonia, dystonia, seizures, respiratory failure, and vomiting. Results obtained in this model system were similar to those of UQCRC1 KD in HeLa cells (Fig. 18). This suggests that the effects observed in HeLa cells are not limited to that model and that a similar mechanism is activated in humans and mice when mitochondria are compromised. Interestingly, the result also showed that irrespective of the perturbed complex of the respiratory chain (complex I in mice and complex III in humans) the impact on cholesterol synthesis was the same. Also, in this case, we could elucidate that the response generated by a mitochondrial malfunction in mice is SREBP1 dependent (Fig. 18B).

The mouse models of LSD that we evaluated in this project corresponded to knock out of three different lysosomal genes in MEFs: Gaa (Fig. 19A) (a model of lysosomal Pompe's disease (Leborgne et al., 2017)), as well as CtsB and CtsL (Fig. 20A). Only three of these disorders can be classified as LSD, while the others are caused by defective cleavage of specific protein substrates (Ketterer et al., 2017). In all the MEF models, we obtained results similar to those that we found in lysosomal KDs in HeLa cells. CtsB and CtsL dysfunction could lead to a similar phenotype observed in Niemann Pick Type C (NPC) cells, where the cholesterol gets accumulated in the Iysosome (Cermak et al., 2016). This could mean that a similar mechanism is employed in all these cases; however, the main reason for this phenotype observed in NPC cells is not well understood, which prevents us from exploring a similar alternative. Consistently, upregulation of most of the genes of the cholesterol synthesis pathway was found in these models, and this increase was SREBP1-dependent (Fig. 20B). Taken together, our results show that similar mechanisms regulate the response to cholesterol biosynthesis in HeLa cells and MEFs. These mechanisms are independent of the enzyme that is inhibited.

The expression levels of the studied genes for the cholesterol synthesis in mouse liver, the most important organ for the de novo synthesis of lipids (Nguyen et al., 2008), followed the same trend observed in the MEF cells (Fig. 21). However, the regulation of the cholesterol pathway in the liver is not only dependent on SREBPs, but can also partially rely on nuclear receptors such as 
liver $X$ receptors (LXRs). These receptors are nuclear proteins that play central roles in the transcriptional control of lipid metabolism; once activated, they induce the synthesis of an array of genes involved in the cholesterol absorption, efflux, transport, and excretion (Zelcer, 2006). Even though we obtained similar results in liver and our models of organelle dysfunction, we cannot conclude that the regulation of the pathway in the tissue is that same as in cells. Further experiments need to be done to clarify if LXRs are critical for the observed regulation in tissue.

\subsubsection{Effect of lysosomal dysfunction in a model of mitochondrial dysfunction and vice versa}

Administration of rotenone to cells produces inhibition of the mitochondrial complex I. The effect of complex I inhibition can be observed in the decrease in oxygen consumption (FernándezMosquera et al., 2017). Besides, it leads to a decrease in the transcript levels of the enzymes involved in cholesterol synthesis (Fig. 22). However, the same treatment does not trigger the decrease in the same transcript levels in CTSB and GAA KD HeLa (Fig. 22 CTSB and GAA). This result suggests that the effect of the lysosomal dysfunction triggers a mechanism of cholesterol synthesis upregulation that suppresses the effect produced by the mitochondrial dysfunction.

On the other hand, when cells with mitochondrial dysfunction are treated with an inhibitor of cholesterol export from the lysosomes, such as U18666A (Eid et al., 2017), the relevant transcript levels are not restored to those observed in control conditions (Fig. 23B). From these results, we can conclude that the effect of the lysosomal dysfunction in the cholesterol pathway has a stronger effect than the one of mitochondrial dysfunction.

The observed results can be explained by analyzing the possible mechanisms in which the cell is able to obtain cholesterol. Lysosomes are essential for the processing of cholesterol absorbed during endocytosis, while mitochondria are organelles important for the production of AcetylCoA, a precursor of most of the lipid molecules. However, Acetyl-CoA can also be produced in the cytoplasm. In the mitochondrial dysfunction model, the cells would not be able to produce the same amount of cholesterol due to a decrease in the production of Acetyl-CoA (Lozoya et al., 2019), but they could use the cholesterol absorbed through endocytosis. If the lysosomes are dysfunctional, the absorption of cholesterol would be hampered, thus triggering an evident upregulation of the cholesterol synthesis pathway.

\subsection{Cholesterol: measurement and reduction}

\subsubsection{Measurement of cholesterol content}

We then evaluated the cellular cholesterol content to verify how it was impacted by the changes in the regulation of cholesterol synthesis. For this purpose, we employed the Filipin stain, which binds to free cholesterol (Fig. 24). 
As we expected, the free content of cholesterol was increased in our models of mitochondrial malfunction and decreased in the case of the lysosomal dysfunction. The ER is the place where the cholesterol levels in the cell are sensed. When the content of cholesterol is lower than $5 \%$, the cholesterol synthesis pathway is activated to restore the cholesterol levels (Brown et al., 2002). So, in the case of the mitochondrial dysfunction, downregulation of the cholesterol pathway is consistent with a higher content of cholesterol in the cell (Fig. 24A). Conversely, the reduced cellular cholesterol content in lysosomal dysfunction models is consistent with the increase in cholesterol biosynthesis (Fig. 24A and B).

The upregulation of the cholesterol biosynthesis pathway in the models of lysosomal malfunction could be explained by the inhibition of cholesterol transport out of the lysosomes. Then, the cholesterol cannot be transported to the ER membrane, and the cells sense its low content (Vance and Karten, 2014). This will, in turn, induce the de novo synthesis, although the lysosomes are full of cholesterol that cannot leave this organelle (Kristiana et al., 2008). In this context, the total content of cholesterol increases due to de novo synthesis and the inability of the cell to use cholesterol storage in the lysosomes.

The free cholesterol measurement by Filipin has several important practical considerations. Filipin is rapidly photobleached by the UV light intensity available in most fluorescence microplate readers (Vanier and Latour, 2015), it can cause deformation in the membrane altering the interaction membrane:protein (Steer et al., 1984), and it can label other lipids such as GM1 ganglioside (Maxfileld and Wüstner, 2012). Due to these concerns, we decided to use a biochemical assay, where the lipids are extracted from the cell, to evaluate total cholesterol content. The lipids are mixed with a cholesterol oxidase enzyme-producing hydrogen peroxide $\left(\mathrm{H}_{2} \mathrm{O}_{2}\right)$. The $\mathrm{H}_{2} \mathrm{O}_{2}$ reacts with a probe forming a new compound that can be easily detected by measuring its fluoresce (Infante and Radhakrishnan, 2017).

Interestingly, the results obtained using this technique were different from those obtained using Filipin. Models of lysosomal dysfunction did not show any change in the cholesterol content (Fig. 25A and B). Nevertheless, the model of mitochondrial dysfunction showed the opposite result, a significant reduction of the cholesterol content (Fig. 25C). The difference in the results could be explained by the ability of Filipin to bind only the unesterified cholesterol (Vanier and Latour, 2015), while the kit allows us also to measure cholesterol esters.

In conditions of some LSDs, e.g., Niemann Pick Type C, it has been reported that there is an increase of the cholesterol levels in the mitochondrial membrane (Charman et al., 2010). For this reason, we decided to measure the cholesterol content also in mitochondria. Gaa KO MEFs show no change (Fig. 26B), but Ndufs4 KO MEFs, there is a significant increase in the mitochondrial cholesterol content. It has been reported that an increase of cholesterol content in the mitochondrial membrane could induce the decrease in the glutathione levels, which results in 
increased ROS levels, further triggering apoptosis (García-Ruiz et al., 2016). Additionally, changes

in the cholesterol content of the mitochondrial membrane influence the mechanism of membrane permeabilization, also leading to apoptosis (Bosch et al., 2011).

\subsubsection{Cholesterol extraction with hydroxypropyl- $\beta$-cyclodextrin (HPCD)}

Using cyclodextrins, cyclic oligosaccharides consisting of multiple glucopyranose water-soluble units along with a hydrophobic pocket, we have performed cholesterol extraction (Zidovetzki and Levitan, 2007). The hydrophobic pocket is the cholesterol-binding site. For example, supplementing medium with 5-10 mM $\beta$-cyclodextrin for more than two hours removes $80-90 \%$ of cellular cholesterol (Yancey et al., 1996). For this reason, we used a milder cyclodextrin, 2Hydroxypropyl- $\beta$-cyclodextrin (HPCD) (Shen et al., 2014).

The addition of HPCD into the medium produced an increase in the transcript levels of the main enzymes involved in the cholesterol biosynthesis in both models of organelle dysfunction. Interestingly, HPCD was able to rescue the effect of the mitochondrial dysfunction on the cholesterol biosynthesis pathway (Fig. 28); sequestration of cholesterol from the cell appears to reactivate the cholesterol biosynthesis despite the mitochondrial dysfunction. The group of Brown (Abi-Mosleh et al., 2009) demonstrated that the treatment with HPCD could rescue the accumulation of cholesterol in the lysosomes observed in NPC1/2 KO human fibroblast. HPCD releases the cholesterol from the lysosomes after which a portion of the released cholesterol can be transferred to ER for esterification. CtsB and CtsL KO MEFs showed the opposite result (Fig. 27): the transcript levels were increased, suggesting an activation of the cholesterol pathway. This experimental difference leads to the conclusion that even if an accumulation of cholesterol is present in CTSB/CTSL deficient cells, the mechanism involved should be different from the NPC cells.

\subsection{Determination of signaling pathways involved}

\subsubsection{Lipid pathway biosynthesis induced by mTORC}

mTORC has an essential function as nutrient/energy/stress sensor. It can induce the lipogenesis in hepatocytes by directly activating SREBPs (Eid et al., 2017). In cells, mTORC1 promotes SREBPs translocation from the ER to Golgi, where SREBPs are cleaved and activated (Peterson et al., 2011). However, it is unclear whether and how mTORC1 influences ER cholesterol to regulate SREBPs (Eid et al., 2017).

For the inhibition of the MTORC pathway, we used TORIN1, an inhibitor of MTORC1 and mTORC2 (Thoreen et al., 2009). MEF cells exposed to TORIN1 show a different response to the effect of the drug. CtsB KO MEFs (Fig. 29B) increased considerably the transcript levels of the enzymes from cholesterol synthesis; on the other hand, there was no change in their transcript levels in Ndufs4 KO MEFs (Fig. 30B). Ndufs4 KO mice, studied as a model of Leigh Syndrome (mitochondrial 
disease), show signs of symptoms alleviation upon the treatment with rapamycin (an inhibitor or mTORC) (Cherezov et al., 2013). As expected in our Ndufs4 KO MEFs, an increase in the activity of mTORC was observed (Fig. 30A). Despite the increase of mTORC activity, which would lead to an increase in the cholesterol synthesis, in Ndufs 4 KO MEF, the synthesis of cholesterol is repressed. This result suggests that the functional complex I, and likely functional mitochondria, are essential to activate cholesterol synthesis and underscores the importance of mitochondrial function in cholesterol metabolism. In summary, the inhibition of mTORC seems not to affect the cholesterol pathway in Ndufs4 KO MEFs.

CTSB regulates lysosomal dynamics. When the enzyme is not present due to a knock out or is pharmacologically inhibited, the activity of $\mathrm{mTORC}$ is reduced, resulting in overt activation of TFEB (Man and Kanneganti, 2016). Despite the reduction in the activity of mTORC, when the activity of CTSB is blocked, we could not observe a reduced activity of MTORC in normal conditions (Fig. 29A). In CtsB KO MEFs, the inhibition of mTORC increased the transcript levels of the main enzymes involved in the cholesterol biosynthesis to the extent higher than in normal conditions. The predicted result based on the previous experiment would have been a reduction in the cholesterol biosynthesis; however, in this case, an opposite result was observed. For this reason, we contemplate the possibility that a different regulator of the pathway is responsible for the increase or decrease of the cholesterol biosynthesis in either mitochondrial or lysosomal dysfunction.

\subsubsection{Lipid pathway biosynthesis repressed by AMPK}

The first pathway ever that was identified as a target of AMPK was the lipid and sterol pathway. AMPK inhibits these pathways through inhibitory phosphorylation of the acetyl-CoA carboxylases ACC1 and ACC2, which catalyze the first step of de novo lipid synthesis, and HMGCR (Carling et al., 1987; Munday et al., 1988).

To identify the role of AMPK in our models of organelle dysfunction, we took advantage of AMPK double KO MEFs, MEF cells lacking the AMPK catalytic subunit $\alpha 1$ and $\alpha 2$. Cells were treated with rotenone and U18666A. Consistent with our expectations, cells lacking AMPK activity in normal conditions showed an increase in the of HMGCS1, HMGCR and MVK transcript levels (Fig. 31 Control) and an increase of active SREBP1 protein levels (Fig. 31). The inactivity of AMPK triggers the cholesterol biosynthesis due to the inhibitory role of AMPK in the lipid synthesis.

The treatment with rotenone (Fig. 31 Rotenone) reproduces the results obtained in our models of mitochondrial dysfunction (Fig. 15 and Fig. 18A). Addition of U18666A increased the relevant transcript levels (Fig. 31 U18666A) in the same way as lysosomal dysfunction. These results confirm that the drug treatments mimic the organelle dysfunction models generated by genetic manipulations. 
Nonetheless, the co-treatment (U18666A and Rotenone, Fig. 31) showed a different trend: WT cells showed an increase in the transcript levels of HMGCS1, HMGCR, and MVK compared with the control WT. The effect of U18666A treatment could not be reverted in any of the KO cell lines. The levels of SREBP1 are not affected by any drug treatment (Fig. 32). This would suggest an essential role of AMPK in the studied mechanism since in the conditions where we could have expected a restoration of the transcript levels to basal values, co-treatment, the cells were not able to restore the normal regulation of cholesterol synthesis in the absence of AMPK. This significant result gave us a hint that AMPK could be essential to explain the differential regulation of the cholesterol pathway in our models of lysosomal and mitochondrial dysfunction.

\subsubsection{Regulation of HMGCR}

To test the hypothesis that differential regulation of AMPK is essential for the differential regulation of the cholesterol synthesis, we decided to measure the AMPK activity in our models. The AMPK activity in CTSB KD HeLa (Fig. 33A), CtsB and CtsL KO MEFs (Fig. 33B) were not changed. Nevertheless, it was previously reported that inhibition of CTSB produced an increase in the AMPK activity (Man and Kanneganti, 2016). GAA KD HeLa (Fig. 33A) showed no significant increase in AMPK activity, but a significant decrease was observed in Gaa KO MEF.

Due to this difference in the AMPK activity between our models of lysosomal dysfunction, we decided to use another model of LSD - Danon disease. The origin of this LSD is associated with a glycogen storage disease due to LAMP2 deficiency (Onyenwoke and Brenman, 2015) and the phenotype is characterized by severe cardiomyopathy and skeletal muscle weakness (Lieberman et al., 2012). LAMP2 has been associated with cholesterol stability and transport itself (Li and Pfeffer, 2016). The transcript levels of the cholesterol pathway in Lamp2 KO MEFs were significantly increased (Fig. 34), and the protein levels of SREBP1 were increased as well (Fig. 37). Most of the lysosomal dysfunction models used in this study were deletions of hydrolytic enzymes, e.g., CTSB and GAA. However, in the case of LAMP2, the protein is a constitutive membrane protein of the lysosome, and the result obtained was consistent with the previously described models: an increase in the main transcript levels of the enzymes involved in the cholesterol biosynthesis and an increase of the protein levels of SREBP1.

In our study, there was a trend to decrease AMPK activity in Lamp2 KO MEFs (Fig. 36). One possible way to inhibit the cholesterol pathway by AMPK is the phosphorylation of HMGCR on Ser871 (Munday et al., 1988). As we expected, the phosphorylation levels of HMGCR were decreased (Fig. 36), supporting our hypothesis of the role of AMPK in the studied mechanism.

To further validate our hypothesis, we measured the AMPK activity in the model of mitochondrial dysfunction, Ndufs4 KO MEFs (Fig. 37B). There was a trend towards increasing the AMPK activity in MEF cells. However, these results are different from those previously reported by our group, where AMPK activity was reduced in brain homogenates of Ndufs 4 KO mice (Fernandez- 
Mosquera et al., 2019). This might be due to the high importance that the cholesterol has for the brain metabolism and since the blood-brain barrier prevents the entry of cholesterol-rich lipoproteins all cholesterol must be made locally (Björkhem and Meaney, 2004). Cholesterol brain metabolism is separated from the rest of the body (Martin et al., 2014).

In the interest of time, we were not able to keep working on our hypothesis concerning the role of AMPK in our models or discover any other signaling pathway affected. Further experiments are required to confirm of the mechanistic details underlying the found pathway. 


\section{SUMMARY AND CONCLUSIONS}

Nowadays, there are several studies done in the field of mitochondrial diseases and lysosomal storage diseases and how these organelle dysfunctions affect the organism, triggering several disorders and pathologies according to the affected tissue. However, how and why these diseases affect the lipid synthesis is not well defined. Therefore, this project was based on understanding the main mechanism involved in cholesterol synthesis.

This thesis describes the effect that mitochondrial dysfunction models (cell lines deficient in UQCRC1 and NDUFS4 (Model of Leigh Syndrome)), and lysosomal storage diseases, (deficiency of lysosomal hydrolases GAA, CTSB, and CTSL or glycoprotein of the membrane, e.g., LAMP2) yield on cholesterol biosynthesis. Taking advantage of the next-generation sequencing, we could observe a differential regulation of the cholesterol pathway between the two organelle dysfunctions. We not only validated the data obtained from NGS, analyzing the transcript levels of the main enzymes involved in the cholesterol pathway but also extended the findings to different models and cell types. Additionally, we reported the importance of the regulation of the pathway by SREBP1 in our models. Despite changes in the activity of SREBP1, according to the studied models of organelle dysfunction, we reported a change of free cholesterol levels, even though the total amount of cholesterol was not altered (except in Ndufs4 KO MEF). To understand the change in the cholesterol content and the activation/inhibition of SREBP1, we evaluated the role of the two main kinases involved in cellular metabolism, mTORC, and AMPK. The results obtained from the inhibition of mTORC do not explain the changes reported in our models. On the other hand, AMPK activity trend to be increased in the mitochondrial models and decreased in our lysosomal models could explain the changes in the cholesterol biosynthesis pathway through the regulatory phosphorylation of HMGCR.

As the conclusion of this thesis project, we could report the possible role of AMPK in the differential regulation of the cholesterol pathway in the models of mitochondria and lysosomal dysfunction. However, further experiments need to be done to finally elucidate and explain the origin of the differential regulation. 


\section{BIBLIOGRAPHY}

Abi-Mosleh, L., Infante, R.E., Radhakrishnan, A., Goldstein, J.L., and Brown, M.S. (2009). Cyclodextrin overcomes deficient lysosome-to-endoplasmic reticulum transport of cholesterol in Niemann-Pick type C cells. Proc. Natl. Acad. Sci. 106, 19316-19321.

Aggarwal, N., and Sloane, B.F. (2014). Cathepsin B: Multiple roles in cancer. PROTEOMICS - Clin. Appl. 8, 427-437. Akram, M. (2014). Citric Acid Cycle and Role of its Intermediates in Metabolism. Cell Biochem. Biophys. 68, 475478.

Alberts, B. (2014). Molecular Biology of The Cell.

Amundson, D.M., and Zhou, M. (1999). Fluorometric method for the enzymatic determination of cholesterol. J. Biochem. Biophys. Methods 38, 43-52.

Amuthan, G., Biswas, G., Ananadatheerthavarada, H.K., Vijayasarathy, C., Shephard, H.M., and Avadhani, N.G. (2002). Mitochondrial stress-induced calcium signaling, phenotypic changes and invasive behavior in human lung carcinoma A549 cells. Oncogene 21, 7839-7849.

Askanas, V., Engel, W.K., DiMauro, S., Brooks, B.R., and Mehler, M. (1976). Adult-Onset Acid Maltase Deficiency. N. Engl. J. Med. 294, 573-578.

Bahl, J.J., Matsuda, M., DeFronzo, R.A., and Bressler, R. (1997). In vitro and in vivo suppression of gluconeogenesis by inhibition of pyruvate carboxylase. Biochem. Pharmacol. 53, 67-74.

Balboa, E., Castro, J., Pinochet, M.-J., Cancino, G.I., Matías, N., José Sáez, P., Martínez, A., Álvarez, A.R., GarciaRuiz, C., Fernandez-Checa, J.C., et al. (2017). MLN64 induces mitochondrial dysfunction associated with increased mitochondrial cholesterol content. Redox Biol. 12, 274-284.

Bandyopadhyay, U., Kaushik, S., Varticovski, L., and Cuervo, A.M. (2008). The Chaperone-Mediated Autophagy Receptor Organizes in Dynamic Protein Complexes at the Lysosomal Membrane. Mol. Cell. Biol. 28, 5747-5763.

Benard, G., Bellance, N., James, D., Parrone, P., Fernandez, H., Letellier, T., and Rossignol, R. (2007). Mitochondrial bioenergetics and structural network organization. J. Cell Sci. 120, 838-848.

Bensinger, S.J., Bradley, M.N., Joseph, S.B., Zelcer, N., Janssen, E.M., Hausner, M.A., Shih, R., Parks, J.S., Edwards, P.A., Jamieson, B.D., et al. (2008). LXR Signaling Couples Sterol Metabolism to Proliferation in the Acquired Immune Response. Cell 134, 97-111.

Bernardi, P., Di Lisa, F., Fogolari, F., and Lippe, G. (2015). From ATP to PTP and Back. Circ. Res. 116, 1850-1862.

Bhosale, G., Sharpe, J.A., Sundier, S.Y., and Duchen, M.R. (2015). Calcium signaling as a mediator of cell energy 
demand and a trigger to cell death. Ann. N. Y. Acad. Sci. 1350, 107-116.

Björkhem, I., and Meaney, S. (2004). Brain Cholesterol: Long Secret Life Behind a Barrier. Arterioscler. Thromb. Vasc. Biol. 24, 806-815.

Bloch, K.E. (1983). Sterol structure and membrane function. CRC Crit. Rev. Biochem. 14, 47-92.

de Bock, C.E., and Thorne, R.F. (2016). A mitochondrial brake on vascular repair. Nature 539, 503-504.

Bonora, M., Wieckowski, M.R., Chinopoulos, C., Kepp, O., Kroemer, G., Galluzzi, L., and Pinton, P. (2015). Molecular mechanisms of cell death: central implication of ATP synthase in mitochondrial permeability transition. Oncogene 34, 1475-1486.

Borena, W., Strohmaier, S., Lukanova, A., Bjørge, T., Lindkvist, B., Hallmans, G., Edlinger, M., Stocks, T., Nagel, G., Manjer, J., et al. (2012). Metabolic risk factors and primary liver cancer in a prospective study of 578,700 adults. Int. J. Cancer 131, 193-200.

Bosch, M., Marí, M., Gross, S.P., Fernández-Checa, J.C., and Pol, A. (2011). Mitochondrial cholesterol: A connection between caveolin, metabolism, and disease. Traffic 12, 1483-1489.

Boyle, J. (2005). Lehninger principles of biochemistry (4th ed.): Nelson, D., and Cox, M. Biochem. Mol. Biol. Educ. 33, 74-75.

Brenner, C., and Moulin, M. (2012). Physiological roles of the permeability transition pore. Circ. Res. 111, 12371247.

Brown, M.S., and Goldstein, J.L. (1986). A receptor-mediated pathway for cholesterol homeostasis. Science 232, 34-47.

Brown, M.S., and Goldstein, J.L. (1997a). The SREBP Pathway: Regulation of Cholesterol Metabolism by Proteolysis of a Membrane-Bound Transcription Factor. Cell 89, 331-340.

Brown, M.S., and Goldstein, J.L. (1997b). The SREBP pathway: Regulation of cholesterol metabolism by proteolysis of a membrane-bound transcription factor. Cell 89, 331-340.

Brown, A.J., Sun, L., Feramisco, J.D., Brown, M.S., and Goldstein, J.L. (2002). Cholesterol addition to ER membranes alters conformation of SCAP, the SREBP escort protein that regulates cholesterol metabolism. Mol. Cell 10, 237245.

Brown, M.S., Radhakrishnan, A., and Goldstein, J.L. (2018). Retrospective on Cholesterol Homeostasis: The Central Role of Scap. Annu. Rev. Biochem. 87, annurev-biochem-062917-011852.

Brown, T.A., Tkachuk, A.N., Shtengel, G., Kopek, B.G., Bogenhagen, D.F., Hess, H.F., and Clayton, D.A. (2011). 
Superresolution fluorescence imaging of mitochondrial nucleoids reveals their spatial range, limits, and membrane interaction. Mol. Cell. Biol. 31, 4994-5010.

Cabello, J., and Marsden, D. (2016). Pompe disease: clinical perspectives. Orphan Drugs Res. Rev. Volume 7, 1-10.

Carling, D., Zammit, V.A., and Hardie, D.G. (1987). A common bicyclic protein kinase cascade inactivates the regulatory enzymes of fatty acid and cholesterol biosynthesis. FEBS Lett. 223, 217-222.

Caron, A., Richard, D., and Laplante, M. (2015). The Roles of mTOR Complexes in Lipid Metabolism. Annu. Rev. Nutr. 35, 321-348.

Carroll, B., and Dunlop, E.A. (2017). The lysosome: a crucial hub for AMPK and mTORC1 signalling. Biochem. J. 474, 1453-1466.

Castellano, B.M., Thelen, A.M., Moldavski, O., Feltes, M., van der Welle, R.E.N., Mydock-McGrane, L., Jiang, X., van Eijkeren, R.J., Davis, O.B., Louie, S.M., et al. (2017). Lysosomal cholesterol activates mTORC1 via an SLC38A9Niemann-Pick C1 signaling complex. Science (80-. ). 355, 1306-1311.

Cermak, S., Kosicek, M., Mladenovic-Djordjevic, A., Smiljanic, K., Kanazir, S., and Hecimovic, S. (2016). Loss of cathepsin B and L leads to lysosomal dysfunction, NPC-like cholesterol sequestration and accumulation of the key Alzheimer's proteins. PLoS One 11, 1-17.

Cerqueira, N.M.F.S.A., Oliveira, E.F., Gesto, D.S., Santos-Martins, D., Moreira, C., Moorthy, H.N., Ramos, M.J., and Fernandes, P.A. (2016). Cholesterol Biosynthesis: A Mechanistic Overview. Biochemistry 55, 5483-5506.

Chae, S., Ahn, B.Y., Byun, K., Cho, Y.M., Yu, M.-H., Lee, B., Hwang, D., and Park, K.S. (2013). A Systems Approach for Decoding Mitochondrial Retrograde Signaling Pathways. Sci. Signal. 6, rs4-rs4.

Chan, D.C. (2012). Fusion and Fission: Interlinked Processes Critical for Mitochondrial Health. Annu. Rev. Genet. 46, 265-287.

Chance, B., and Williams, G.R. (2006). The Respiratory Chain and Oxidative Phosphorylation. pp. 65-134.

Chandel, N.S. (2015). Evolution of Mitochondria as Signaling Organelles. Cell Metab. 22, $204-206$.

Charman, M., Kennedy, B.E., Osborne, N., and Karten, B. (2010). MLN64 mediates egress of cholesterol from endosomes to mitochondria in the absence of functional Niemann-Pick Type C1 protein. J. Lipid Res. 51, 10231034.

Cherezov, V., Stevens, R.C., Bouvier, M., Rosenbusch, J.P., Li, D., Dukkipati, A., Fischetti, R.F., Yamamoto, M., Williams, G.J., Pryor, A.W., et al. (2013). mTOR Inhibition Alleviates Mitochondrial Disease in a Mouse Model of Leigh Syndrome. Science (80-. ). 342, 1524-1529. 
Chinopoulos, C., and Szabadkai, G. (2014). What Makes You Can also Break You, Part III: Mitochondrial Permeability Transition Pore Formation by an Uncoupling Channel within the C-Subunit Ring of the F1FO ATP Synthase? Front. Oncol. 4, 235.

Clayton, D.A. (1982). Replication of animal mitochondrial DNA. Cell 28, 693-705.

Clendening, J.W., Pandyra, A., Boutros, P.C., El Ghamrasni, S., Khosravi, F., Trentin, G.A., Martirosyan, A., Hakem, A., Hakem, R., Jurisica, I., et al. (2010). Dysregulation of the mevalonate pathway promotes transformation. Proc. Natl. Acad. Sci. U. S. A. 107, 15051-15056.

Cogliati, S., Frezza, C., Soriano, M.E., Varanita, T., Quintana-Cabrera, R., Corrado, M., Cipolat, S., Costa, V., Casarin, A., Gomes, L.C., et al. (2013). Mitochondrial cristae shape determines respiratory chain supercomplexes assembly and respiratory efficiency. Cell 155, 160-171.

Cogliati, S., Enriquez, J.A., and Scorrano, L. (2016). Mitochondrial Cristae: Where Beauty Meets Functionality. Trends Biochem. Sci. 41, 261-273.

Cooper, G.M. (2000). The cell : a molecular approach (ASM Press).

Cooper, R.A., and Shattil, S.J. (1980). Membrane Cholesterol - Is Enough Too Much? N. Engl. J. Med. 302, $49-51$. Cuervo, A.M., and Dice, J.F. (1996). A Receptor for the Selective Uptake and Degradation of Proteins by Lysosomes. Science (80-. ). 273, 501-503.

DeBerardinis, R.J., and Cheng, T. (2010). Q's next: the diverse functions of glutamine in metabolism, cell biology and cancer. Oncogene 29, 313-324.

Devanathan, G., Turnbull, J.L., Ziomek, E., Purisima, E.O., Ménard, R., and Sulea, T. (2005). Carboxymonopeptidase substrate specificity of human cathepsin X. Biochem. Biophys. Res. Commun. 329, 445-452.

Dowhan, W. (1997). MOLECULAR BASIS FOR MEMBRANE PHOSPHOLIPID DIVERSITY:Why Are There So Many Lipids? Annu. Rev. Biochem. 66, 199-232.

Ducker, G.S., and Rabinowitz, J.D. (2017). One-Carbon Metabolism in Health and Disease. Cell Metab. 25, $27-42$. de Duve, C. (1983). Lysosomes revisited. Eur. J. Biochem. 137, 391-397.

de Duve, C. (2005). The lysosome turns fifty. Nat. Cell Biol. 7, 847-849.

de Duve, C., Pressman, B.C., Gianetto, R., Wattiaux, R., and Appelmans, F. (1955). Tissue fractionation studies. 6. Intracellular distribution patterns of enzymes in rat-liver tissue. Biochem. J. 60, 604-617.

Ebner, M., Sinkovics, B., Szczygiet, M., Ribeiro, D.W., and Yudushkin, I. (2017). Localization of mTORC2 activity inside cells. J. Cell Biol. 216, 343-353. 
Egan, D.F., Shackelford, D.B., Mihaylova, M.M., Gelino, S., Kohnz, R.A., Mair, W., Vasquez, D.S., Joshi, A., Gwinn, D.M., Taylor, R., et al. (2011). Phosphorylation of ULK1 (hATG1) by AMP-Activated Protein Kinase Connects Energy Sensing to Mitophagy. Science (80-. ). 331, 456-461.

Eid, W., Dauner, K., Courtney, K.C., Gagnon, A., Parks, R.J., Sorisky, A., and Zha, X. (2017). mTORC1 activates SREBP-2 by suppressing cholesterol trafficking to lysosomes in mammalian cells. Proc. Natl. Acad. Sci. 114, 79998004.

El-Hattab, A.W., and Scaglia, F. (2016). Mitochondrial Cardiomyopathies. Front. Cardiovasc. Med. 3, 1-9.

Ernster, L., and Schatz, G. (1981). Mitochondria : A Historical Review. 91.

Felbor, U., Kessler, B., Mothes, W., Goebel, H.H., Ploegh, H.L., Bronson, R.T., and Olsen, B.R. (2002). Neuronal loss and brain atrophy in mice lacking cathepsins B and L. Proc. Natl. Acad. Sci. 99, 7883-7888.

Fernandez-Mosquera, L., Yambire, K.F., Couto, R., Pereyra, L., Pabis, K., Ponsford, A.H., Diogo, C. V., Stagi, M., Milosevic, I., and Raimundo, N. (2019). Mitochondrial respiratory chain deficiency inhibits lysosomal hydrolysis. Autophagy 0, 1-20.

Fernández-Mosquera, L., Diogo, C. V., Yambire, K.F., Santos, G.L., Luna Sánchez, M., Bénit, P., Rustin, P., Lopez, L.C., Milosevic, I., and Raimundo, N. (2017). Acute and chronic mitochondrial respiratory chain deficiency differentially regulate lysosomal biogenesis. Sci. Rep. 7, 45076.

Filadi, R., Pendin, Di., and Pizzo, P. (2018). Mitofusin 2: From functions to disease. Cell Death Dis. 9.

Folch, J., Lees, M., and Sloane Stanley, G.H. (1957). A simple method for the isolation and purification of total lipides from animal tissues. J. Biol. Chem. 226, 497-509.

Fonović, M., and Turk, B. (2014). Cysteine cathepsins and extracellular matrix degradation. Biochim. Biophys. Acta - Gen. Subj. 1840, 2560-2570.

Formentini, L., Sánchez-Aragó, M., Sánchez-Cenizo, L., and Cuezva, J.M. (2012). The Mitochondrial ATPase Inhibitory Factor 1 Triggers a ROS-Mediated Retrograde Prosurvival and Proliferative Response. Mol. Cell 45, 731742.

Frey, T.G., and Mannella, C.A. (2000). The internal structure of mitochondria. Trends Biochem. Sci. $0004,319-324$. Friedman, J.R., and Nunnari, J. (2014). Mitochondrial form and function. Nature 505, 335-343.

Fuller, M., Meikle, P.J., and Hopwood, J.J. (2006). Epidemiology of lysosomal storage diseases: an overview.

Futerman, A.H., and Van Meer, G. (2004). The cell biology of lysosomal storage disorders. Nat. Rev. Mol. Cell Biol. 5, 554-565. 
Garcia-Roves, P.M., Osler, M.E., Holmström, M.H., and Zierath, J.R. (2008). Gain-of-function R225Q mutation in AMP-activated protein kinase $\gamma 3$ subunit increases mitochondrial biogenesis in glycolytic skeletal muscle. J. Biol. Chem. 283, 35724-35734.

García-Ruiz, C., Ribas, V., Baulies, A., and Fernández-Checa, J.C. (2016). Mitochondrial Cholesterol and the Paradox in Cell Death. pp. 1-22.

Ghosh, P., Dahms, N.M., and Kornfeld, S. (2003). Mannose 6-phosphate receptors: new twists in the tale. Nat. Rev. Mol. Cell Biol. 4, 202-213.

Goldstein, J.L., and Brown, M.S. (2015). A Century of Cholesterol and Coronaries: From Plaques to Genes to Statins. Cell 161, 161-172.

Goldstein, J.L., DeBose-Boyd, R.A., and Brown, M.S. (2006). Protein sensors for membrane sterols. Cell 124, 35-46.

Gorman, G.S., Schaefer, A.M., Ng, Y., Gomez, N., Blakely, E.L., Alston, C.L., Feeney, C., Horvath, R., Yu-Wai-Man, P., Chinnery, P.F., et al. (2015). Prevalence of nuclear and mitochondrial DNA mutations related to adult mitochondrial disease. Ann. Neurol. 77, 753-759.

Gorman, G.S., Chinnery, P.F., DiMauro, S., Hirano, M., Koga, Y., McFarland, R., Suomalainen, A., Thorburn, D.R., Zeviani, M., and Turnbull, D.M. (2016). Mitochondrial diseases. Nat. Rev. Dis. Prim. 2, 16080.

Griffin, J.L. (1984). Infantile acid maltase deficiency. I. Muscle fiber destruction after lysosomal rupture. Virchows Arch. B. Cell Pathol. Incl. Mol. Pathol. 45, 23-36.

Gustafsson, C.M., Falkenberg, M., and Larsson, N.-G. (2016). Maintenance and Expression of Mammalian Mitochondrial DNA. Annu. Rev. Biochem. 85, 133-160.

Haack, T.B., Haberberger, B., Frisch, E.M., Wieland, T., Iuso, A., Gorza, M., Strecker, V., Graf, E., Mayr, J.A., Herberg, U., et al. (2012). Molecular diagnosis in mitochondrial complex I deficiency using exome sequencing. J. Med. Genet. 49, 277-283.

Hällberg, B.M., and Larsson, N.G. (2014). Making proteins in the powerhouse. Cell Metab. 20, $226-240$.

Hamanaka, R.B., and Chandel, N.S. (2010). Mitochondrial reactive oxygen species regulate cellular signaling and dictate biological outcomes. Trends Biochem. Sci. 35, 505-513.

Han, Y., Hu, Z., Cui, A., Liu, Z., Ma, F., Xue, Y., Liu, Y., Zhang, F., Zhao, Z., Yu, Y., et al. (2019). Post-translational regulation of lipogenesis via AMPK-dependent phosphorylation of insulin-induced gene. Nat. Commun. $10,1-13$.

Hansen, M., Rubinsztein, D.C., and Walker, D.W. (2018). Autophagy as a promoter of longevity: insights from model organisms. Nat. Rev. Mol. Cell Biol. 19, 579-593. 
Hardie, D.G. (2007). AMP-activated/SNF1 protein kinases: conserved guardians of cellular energy. Nat. Rev. Mol. Cell Biol. 8, 774-785.

Hardie, D.G., Carling, D., and Carlson, M. (1998). THE AMP-ACTIVATED/SNF1 PROTEIN KINASE SUBFAMILY: Metabolic Sensors of the Eukaryotic Cell? Annu. Rev. Biochem. 67, 821-855.

Hatzivassiliou, G., Zhao, F., Bauer, D.E., Andreadis, C., Shaw, A.N., Dhanak, D., Hingorani, S.R., Tuveson, D.A., and Thompson, C.B. (2005). ATP citrate lyase inhibition can suppress tumor cell growth. Cancer Cell 8, 311-321.

Heiden, M.G. Vander, Cantley, L.C., and Thompson, C.B. (2009). Understanding the warburg effect: The metabolic requirements of cell proliferation. Science (80-. ). 324, 1029-1033.

Herzig, S., and Shaw, R.J. (2018). AMPK: Guardian of metabolism and mitochondrial homeostasis. Nat. Rev. Mol. Cell Biol. 19, 121-135.

Holt, I.J., Lorimer, H.E., and Jacobs, H.T. (2000). Coupled Leading-and Lagging-Strand Synthesis of Mammalian Mitochondrial DNA eubacteria. Replication of the $\mathrm{H}$ strand on this model is proposed to initiate at a single site $(O$ $\mathrm{H}$ ), and to proceed unidirectionally until two-thirds of the way around the These.

Holtzman, E. (1989). Lysosomes (Boston, MA: Springer US).

Hoppins, S., Lackner, L., and Nunnari, J. (2007). The Machines that Divide and Fuse Mitochondria. Annu. Rev. Biochem. 76, 751-780.

Horton, J.D., Goldstein, J.L., and Brown, M.S. (2002). SREBPs: activators of the complete program of cholesterol and fatty acid synthesis in the liver. J. Clin. Invest. 109, 1125-1131.

Hsu, V.W., and Prekeris, R. (2010). Transport at the recycling endosome. Curr. Opin. Cell Biol. 22, 528-534.

Hu, Y.-B., Dammer, E.B., Ren, R.-J., and Wang, G. (2015). The endosomal-lysosomal system: from acidification and cargo sorting to neurodegeneration. Transl. Neurodegener. 4, 18.

Hua, X., Nohturfft, A., Goldstein, J.L., and Brown, M.S. (1996). Sterol Resistance in CHO Cells Traced to Point Mutation in SREBP Cleavage-Activating Protein. Cell 87, 415-426.

Huotari, J., and Helenius, A. (2011). Endosome maturation. EMBO J. 30, 3481-3500.

Huynh, K.K., Eskelinen, E., Scott, C.C., Malevanets, A., Saftig, P., and Grinstein, S. (2007). LAMP proteins are required for fusion of lysosomes with phagosomes. EMBO J. 26, 313-324.

Hyman, A.H., and Simons, K. (2011). Beyond HeLa cells. Nature 480, 34-34.

Infante, R.E., and Radhakrishnan, A. (2017). Continuous transport of a small fraction of plasma membrane cholesterol to endoplasmic reticulum regulates total cellular cholesterol. Elife 6, 1-23. 
Inoki, K., Kim, J., and Guan, K.-L. (2011). AMPK and mTOR in Cellular Energy Homeostasis and Drug Targets. Annu. Rev. Pharmacol. Toxicol. 52, 381-400.

Ishibashi, O., Mori, Y., Kurokawa, T., and Kumegawa, M. (1999). Breast cancer cells express cathepsins B and L but not cathepsins $\mathrm{K}$ or H. Cancer Biochem. Biophys. 17, 69-78.

Jaishy, B., and Abel, E.D. (2016). Lipids, lysosomes, and autophagy. J. Lipid Res. 57, 1619-1635.

Jastrzebski, K., Hannan, K.M., Tchoubrieva, E.B., Hannan, R.D., and Pearson, R.B. (2007). Coordinate regulation of ribosome biogenesis and function by the ribosomal protein S6 kinase, a key mediator of mTOR function. Growth Factors 25, 209-226.

Jazwinski, S.M. (2013). The retrograde response: When mitochondrial quality control is not enough. Biochim. Biophys. Acta - Mol. Cell Res. 1833, 400-409.

Kanayama, T., Arito, M., So, K., Hachimura, S., Inoue, J., and Sato, R. (2007). Interaction between Sterol Regulatory Element-binding Proteins and Liver Receptor Homolog-1 Reciprocally Suppresses Their Transcriptional Activities. J. Biol. Chem. 282, 10290-10298.

Kaushik, S., and Cuervo, A.M. (2018). The coming of age of chaperone-mediated autophagy. Nat. Rev. Mol. Cell Biol. 19, 365-381.

Kelly, D.P. (2004). Transcriptional regulatory circuits controlling mitochondrial biogenesis and function. Genes Dev. 18, 357-368.

Ketterer, S., Gomez-Auli, A., Hillebrand, L.E., Petrera, A., Ketscher, A., and Reinheckel, T. (2017). Inherited diseases caused by mutations in cathepsin protease genes. FEBS J. 284, 1437-1454.

Kilpatrick, B.S., Eden, E.R., Hockey, L.N., Futter, C.E., and Patel, S. (2015). Methods for monitoring lysosomal morphology (Elsevier Ltd).

Kim, J., and Guan, K.-L. (2019). mTOR as a central hub of nutrient signalling and cell growth. Nat. Cell Biol. 21, 6371.

Kim, J., Yang, G., Kim, Y., Kim, J., and Ha, J. (2016). AMPK activators: mechanisms of action and physiological activities. Exp. Mol. Med. 48, e224.

Kolter, T., and Sandhoff, K. (2005). PRINCIPLES OF LYSOSOMAL MEMBRANE DIGESTION: Stimulation of Sphingolipid Degradation by Sphingolipid Activator Proteins and Anionic Lysosomal Lipids. Annu. Rev. Cell Dev. Biol. 21, 81-103.

Kolter, T., and Sandhoff, K. (2010). Lysosomal degradation of membrane lipids. FEBS Lett. 584, $1700-1712$. 
Koshiba, T., Detmer, S.A., Kaiser, J.T., Chen, H., McCaffery, J.M., and Chan, D.C. (2004). Structural basis of mitochondrial tethering by mitofusin complexes. Science (80-. ). 305, 858-862.

Kristiana, I., Yang, H., and Brown, A.J. (2008). Different kinetics of cholesterol delivery to components of the cholesterol homeostatic machinery: Implications for cholesterol trafficking to the endoplasmic reticulum. Biochim. Biophys. Acta - Mol. Cell Biol. Lipids 1781, 724-730.

Kroos, M.A., Mullaart, R.A., Van Vliet, L., Pomponio, R.J., Amartino, H., Kolodny, E.H., Pastores, G.M., Wevers, R.A., Van der Ploeg, A.T., Halley, D.J.J., et al. (2008). p.[G576S; E689K]: pathogenic combination or polymorphism in Pompe disease? Eur. J. Hum. Genet. 16, 875-879.

Krycer, J.R., Sharpe, L.J., Luu, W., and Brown, A.J. (2010). The Akt-SREBP nexus: cell signaling meets lipid metabolism. Trends Endocrinol. Metab. 21, 268-276.

Kühl, I., Miranda, M., Atanassov, I., Kuznetsova, I., Hinze, Y., Mourier, A., Filipovska, A., and Larsson, N.G. (2017). Transcriptomic and proteomic landscape of mitochondrial dysfunction reveals secondary coenzyme $Q$ deficiency in mammals. Elife 6, 1-33.

Kukat, C., Wurm, C.A., Spahr, H., Falkenberg, M., Larsson, N.-G., and Jakobs, S. (2011). Super-resolution microscopy reveals that mammalian mitochondrial nucleoids have a uniform size and frequently contain a single copy of mtDNA. Proc. Natl. Acad. Sci. 108, 13534-13539.

Kumari, S., MG, S., and Mayor, S. (2010). Endocytosis unplugged: multiple ways to enter the cell. Cell Res. 20, 256275.

Laifenfeld, D., Patzek, L.J., McPhie, D.L., Chen, Y., Levites, Y., Cataldo, A.M., and Neve, R.L. (2007). Rab5 Mediates an Amyloid Precursor Protein Signaling Pathway That Leads to Apoptosis. J. Neurosci. 27, 7141-7153.

Lake, N.J., Compton, A.G., Rahman, S., and Thorburn, D.R. (2016). Leigh syndrome: One disorder, more than 75 monogenic causes. Ann. Neurol. 79, 190-203.

Larsson, N.-G., Wang, J., Wilhelmsson, H., Oldfors, A., Rustin, P., Lewandoski, M., Barsh, G.S., and Clayton, D.A. (1998). Mitochondrial transcription factor $A$ is necessary for mtDNA maintance and embryogenesis in mice. Nat. Genet. 18, 231-236.

Lawrence, R.E., and Zoncu, R. (2019). The lysosome as a cellular centre for signalling, metabolism and quality control. Nat. Cell Biol. 21, 133-142.

Leborgne, C., Barkats, M., Mingozzi, F., Kay, M.A., Puzzo, F., Hardet, R., Collaud, F., Boisgerault, F., Gjata, B., Vidal, P., et al. (2017). Rescue of Pompe disease in mice by AAV-mediated liver delivery of secretable acid $\alpha$-glucosidase. Sci. Transl. Med. 9, eaam6375. 
Li, J., and Pfeffer, S.R. (2016). Lysosomal membrane glycoproteins bind cholesterol and contribute to lysosomal cholesterol export. Elife 5, 1-16.

Li, W.W., Li, J., and Bao, J.K. (2012). Microautophagy: Lesser-known self-eating. Cell. Mol. Life Sci. 69, 1125-1136. Li, X., Gu, J., and Zhou, Q. (2015). Review of aerobic glycolysis and its key enzymes - new targets for lung cancer therapy. Thorac. Cancer 6, 17-24.

Li, Y., Xu, S., Mihaylova, M.M., Zheng, B., Hou, X., Jiang, B., Park, O., Luo, Z., Lefai, E., Shyy, J.Y.J., et al. (2011). AMPK phosphorylates and inhibits SREBP activity to attenuate hepatic steatosis and atherosclerosis in dietinduced insulin-resistant mice. Cell Metab. 13, 376-388.

Lieberman, A.P., Puertollano, R., Raben, N., Slaugenhaupt, S., Walkley, S.U., and Ballabio, A. (2012). Autophagy in lysosomal storage disorders. Autophagy 8, 719-730.

Lightowlers, R.N., Taylor, R.W., and Turnbull, D.M. (2015). Mutations causing mitochondrial disease: What is new and what challenges remain? Science (80-. ). 349, 1494-1499.

Lim, J.-A., Li, L., Kakhlon, O., Myerowitz, R., and Raben, N. (2015). Defects in calcium homeostasis and mitochondria can be reversed in Pompe disease. Autophagy 11, 385-402.

Loewith, R., Jacinto, E., Wullschleger, S., Lorberg, A., Crespo, J.L., Bonenfant, D., Oppliger, W., Jenoe, P., and Hall, M.N. (2002). Two TOR complexes, only one of which is rapamycin sensitive, have distinct roles in cell growth control. Mol. Cell 10, 457-468.

Lozoya, O.A., Wang, T., Grenet, D., Wolfgang, T.C., Sobhany, M., Ganini da Silva, D., Riadi, G., Chandel, N., Woychik, R.P., and Santos, J.H. (2019). Mitochondrial acetyl-CoA reversibly regulates locus-specific histone acetylation and gene expression. Life Sci. Alliance 2, e201800228.

Man, S.M., and Kanneganti, T.D. (2016). Regulation of lysosomal dynamics and autophagy by CTSB/cathepsin B. Autophagy 12, 2504-2505.

Mannella, C.A. (2006). Structure and dynamics of the mitochondrial inner membrane cristae. Biochim. Biophys. Acta - Mol. Cell Res. 1763, 542-548.

Martin, M.G., Pfrieger, F., and Dotti, C.G. (2014). Cholesterol in brain disease: sometimes determinant and frequently implicated. EMBO Rep. 15, 1036-1052.

Martina, J.A., Diab, H.I., Brady, O.A., and Puertollano, R. (2016). TFEB and TFE3 are novel components of the integrated stress response. EMBO J. 35, 479-495.

Maxfield, F.R., and Tabas, I. (2005). Role of cholesterol and lipid organization in disease. Nature 438, 612-621. 
Maxfileld, F., and Wüstner, D. (2012). Analysis of cholesterol trafficking with fluorescent probes. Methods Cell Biol 108, 367-393.

McFarland, R., Taylor, R.W., and Turnbull, D.M. (2010). A neurological perspective on mitochondrial disease. Lancet Neurol. 9, 829-840.

Meeusen, S., McCaffery, J.M., and Nunnari, J. (2004). Mitochondrial Fusion Intermediates Revealed in Vitro. Science (80-. ). 305, 1747-1752.

Merrill, A.H. (2013). Thematic Review Series on the Living History of Lipids. J. Lipid Res. 54, $2945-2945$.

Mindell, J.A. (2012). Lysosomal Acidification Mechanisms. Annu. Rev. Physiol. 74, 69-86.

Mohamed, A., Smith, K., and de Chaves, E.P. (2015). The Mevalonate Pathway in Alzheimer's Disease Cholesterol and Non-Sterol Isoprenoids. In Alzheimer's Disease - Challenges for the Future, (InTech), p. 64.

Munday, M.R., Campbell, D.G., Carling, D., and Herdie, D.G. (1988). Identification by amino acid sequencing of three major regulatory phosphorylation sites on rat acetyl-CoA carboxylase. Eur. J. Biochem. 175, 331-338.

Murdoch, J.D., Rostosky, C.M., Gowrisankaran, S., Arora, A.S., Soukup, S.F., Vidal, R., Capece, V., Freytag, S., Fischer, A., Verstreken, P., et al. (2016). Endophilin-A Deficiency Induces the Foxo3a-Fbxo32 Network in the Brain and Causes Dysregulation of Autophagy and the Ubiquitin-Proteasome System. Cell Rep. 17, 1071-1086.

Muro, E., Atilla-Gokcumen, G.E., and Eggert, U.S. (2014). Lipids in cell biology: how can we understand them better? Mol. Biol. Cell 25, 1819-1823.

Murphy, M.P. (2009). How mitochondria produce reactive oxygen species. Biochem. J. 417, 1-13.

Nägler, D.K., Zhang, R., Tam, W., Sulea, T., Purisima, E.O., and Ménard, R. (1999). Human Cathepsin X: A Cysteine Protease with Unique Carboxypeptidase Activity t. Biochemistry 38, 12648-12654.

Nakada, K., Inoue, K., Ono, T., Isobe, K., Ogura, A., Goto, Y.I., Nonaka, I., and Hayashi, J.I. (2001). Intermitochondrial complementation: Mitochondria-specific system preventing mice from expression of disease phenotypes by mutant mtDNA. Nat. Med. 7, 934-940.

Napolitano, G., and Ballabio, A. (2016). TFEB at a glance. J. Cell Sci. 129, 2475-2481.

Nazaret, C., Heiske, M., Thurley, K., and Mazat, J.-P. (2009). Mitochondrial energetic metabolism: A simplified model of TCA cycle with ATP production. J. Theor. Biol. 258, 455-464.

Needham, D., and Nunn, R.S. (1990). Elastic deformation and failure of lipid bilayer membranes containing cholesterol. Biophys. J. 58, 997-1009.

Nguyen, P., Leray, V., Diez, M., Serisier, S., Le Bloc'H, J., Siliart, B., and Dumon, H. (2008). Liver lipid metabolism. J. 
Anim. Physiol. Anim. Nutr. (Berl). 92, 272-283.

Nohturfft, A., Brown, M.S., and Goldstein, J.L. (1998). Sterols regulate processing of carbohydrate chains of wildtype SREBP cleavage-activating protein (SCAP), but not sterol-resistant mutants Y298C or D443N. Proc. Natl. Acad. Sci. U. S. A. 95, 12848-12853.

Nunnari, J., and Suomalainen, A. (2012). Mitochondria: In Sickness and in Health. Cell 148, 1145-1159.

Ohvo-Rekilä, H., Ramstedt, B., Leppimäki, P., and Peter Slotte, J. (2002). Cholesterol interactions with phospholipids in membranes. Prog. Lipid Res. 41, 66-97.

Olson, O.C., and Joyce, J.A. (2015). Cysteine cathepsin proteases: regulators of cancer progression and therapeutic response. Nat. Rev. Cancer 15, 712-729.

Onyenwoke, R.U., and Brenman, J.E. (2015). Lysosomal Storage Diseases-Regulating Neurodegeneration Supplementary Issue: Molecular and Cellular Mechanisms of Neurodegeneration. J. Exp. Neurosci. 2015, 81-91.

Ortigoza-Escobar, J.D., Oyarzabal, A., Montero, R., Artuch, R., Jou, C., Jiménez, C., Gort, L., Briones, P., Muchart, J., López-Gallardo, E., et al. (2016). Ndufs4 related Leigh syndrome: A case report and review of the literature. Mitochondrion 28, 73-78.

Parenti, G., Pignata, C., Vajro, P., and Salerno, M. (2013). New strategies for the treatment of lysosomal storage diseases (Review). Int. J. Mol. Med. 31, 11-20.

Parenti, G., Andria, G., and Ballabio, A. (2015). Lysosomal Storage Diseases: From Pathophysiology to Therapy. Annu. Rev. Med. 66, 471-486.

Patel, S., and Docampo, R. (2010). Acidic calcium stores open for business: expanding the potential for intracellular Ca2+ signaling. Trends Cell Biol. 20, 277-286.

Perera, R.M., and Zoncu, R. (2016). The Lysosome as a Regulatory Hub. Annu. Rev. Cell Dev. Biol. 32, $223-253$.

Pernas, L., and Scorrano, L. (2016). Mito-Morphosis: Mitochondrial Fusion, Fission, and Cristae Remodeling as Key Mediators of Cellular Function. Annu. Rev. Physiol. 78, 505-531.

Peterson, T.R., Sengupta, S.S., Harris, T.E., Carmack, A.E., Kang, S.A., Balderas, E., Guertin, D.A., Madden, K.L., Carpenter, A.E., Finck, B.N., et al. (2011). mTOR Complex 1 Regulates Lipin 1 Localization to Control the SREBP Pathway. Cell 146, 408-420.

Picca, A., and Lezza, A.M.S. (2015). Regulation of mitochondrial biogenesis through TFAM-mitochondrial DNA interactions. Useful insights from aging and calorie restriction studies. Mitochondrion 25, 67-75.

Pillay, C.S., Elliott, E., and Denninson, C. (2002). Endolysosomal proteolysis and its regulation. Biochem. J. $363,417$. 
Porstmann, T., Santos, C.R., Griffiths, B., Cully, M., Wu, M., Leevers, S., Griffiths, J.R., Chung, Y.-L., and Schulze, A. (2008). SREBP Activity Is Regulated by mTORC1 and Contributes to Akt-Dependent Cell Growth. Cell Metab. 8, 224236.

Poteryaev, D., Datta, S., Ackema, K., Zerial, M., and Spang, A. (2010). Identification of the Switch in Early-to-Late Endosome Transition. Cell 141, 497-508.

Qiu, L., Lai, W., Stumpo, D., and Blackshear, P. (2016). Mouse Embryonic Fibroblast Cell Culture and Stimulation. Bio-Protocol 6.

Raben, N., Baum, R., Schreiner, C., Takikita, S., Mizushima, N., Ralston, E., and Plotz, P.H. (2009). When more is less: Excess and deficiency of autophagy coexist in skeletal muscle in Pompe disease. Autophagy 5, $111-113$.

Radhakrishnan, A., Goldstein, J.L., McDonald, J.G., and Brown, M.S. (2008). Switch-like Control of SREBP-2 Transport Triggered by Small Changes in ER Cholesterol: A Delicate Balance. Cell Metab. 8, 512-521.

Raimundo, N. (2014). Mitochondrial pathology: Stress signals from the energy factory. Trends Mol. Med. 20, 282292.

Rambold, A.S., Kostelecky, B., Elia, N., and Lippincott-Schwartz, J. (2011). Tubular network formation protects mitochondria from autophagosomal degradation during nutrient starvation. Proc. Natl. Acad. Sci. 108, 1019010195.

Ribas, V., García-Ruiz, C., and Fernández-Checa, J.C. (2016). Mitochondria, cholesterol and cancer cell metabolism. Clin. Transl. Med. 5, 22.

Risley, J.M. (2009). Cholesterol Biosynthesis: Lanosterol to Cholesterol. J. Chem. Educ. 79, 377.

Rizzuto, R., De Stefani, D., Raffaello, A., and Mammucari, C. (2012). Mitochondria as sensors and regulators of calcium signalling. Nat. Rev. Mol. Cell Biol. 13, 566-578.

Robberson, D.L., and Clayton, D.A. (1972). Replication of Mitochondrial DNA in Mouse L Cells and Their Thymidine Kinase-Derivatives: Displacement Replication on a Covalently-Closed Circular Template (electron microscopy/mouse DNA/density gradient centrifugation).

Robitaille, A.M., Christen, S., Shimobayashi, M., Cornu, M., Fava, L.L., Moes, S., Prescianotto-Baschong, C., Sauer, U., Jenoe, P., and Hall, M.N. (2013). Quantitative Phosphoproteomics Reveal mTORC1 Activates de Novo Pyrimidine Synthesis. Science (80-. ). 339, 1320-1323.

Röhrig, F., and Schulze, A. (2016). The multifaceted roles of fatty acid synthesis in cancer. Nat. Rev. Cancer 16, 732-749.

Saftig, P., and Klumperman, J. (2009). Lysosome biogenesis and lysosomal membrane proteins: trafficking meets 
function. Nat. Rev. Mol. Cell Biol. 10, 623-635.

Sanz, P., Rubio, T., and Garcia-Gimeno, M.A. (2013). AMPKbeta subunits: More than just a scaffold in the formation of AMPK complex. FEBS J. 280, 3723-3733.

Lo Sasso, G., Celli, N., Caboni, M., Murzilli, S., Salvatore, L., Morgano, A., Vacca, M., Pagliani, T., Parini, P., and Moschetta, A. (2010). Down-regulation of the LXR transcriptome provides the requisite cholesterol levels to proliferating hepatocytes. Hepatology 51, 1334-1344.

Settembre, C., De Cegli, R., Mansueto, G., Saha, P.K., Vetrini, F., Visvikis, O., Huynh, T., Carissimo, A., Palmer, D., Jürgen Klisch, T., et al. (2013). TFEB controls cellular lipid metabolism through a starvation-induced autoregulatory loop. Nat. Cell Biol. 15, 647-658.

Shao, W., and Espenshade, P.J. (2012). Expanding Roles for SREBP in Metabolism. Cell Metab. 16, $414-419$.

Sharpe, L.J., and Brown, A.J. (2013). Controlling cholesterol synthesis beyond 3-hydroxy-3-methylglutaryl-CoA reductase (HMGCR). J. Biol. Chem. 288, 18707-18715.

Shen, H.-M., and Mizushima, N. (2014). At the end of the autophagic road: an emerging understanding of lysosomal functions in autophagy. Trends Biochem. Sci. 39, 61-71.

Shen, H., Giordano, F., Wu, Y., Chan, J., Zhu, C., Milosevic, I., Wu, X., Yao, K., Chen, B., Baumgart, T., et al. (2014). Coupling between endocytosis and sphingosine kinase 1 recruitment. Nat. Cell Biol. 16, 652-662.

Shimano, H., and Sato, R. (2017). SREBP-regulated lipid metabolism: convergent physiology - divergent pathophysiology. Nat. Publ. Gr.

Shimomura, I., Bashmakov, Y., Ikemoto, S., Horton, J.D., Brown, M.S., and Goldstein, J.L. (1999). Insulin selectively increases SREBP-1c MRNA in the livers of rats with streptozotocin-induced diabetes. Proc. Natl. Acad. Sci. 96, 13656-13661.

Shoshan-Barmatz, V., De Pinto, V., Zweckstetter, M., Raviv, Z., Keinan, N., and Arbel, N. (2010). VDAC, a multifunctional mitochondrial protein regulating cell life and death. Mol. Aspects Med. 31, 227-285.

Singer, S.J., and Nicolson, G.L. (1972). The Fluid Mosaic Model of the Structure of Cell Membranes. Source Sci. New Ser. 175, 720-731.

Steer, C.J., Bisher, M., Blumenthal, R., and Steven, A.C. (1984). Detection of membrane cholesterol by filipin in isolated rat liver coated vesicles is dependent upon removal of the clathrin coat. J. Cell Biol. 99, 315-319.

Suomalainen, A., and Battersby, B.J. (2018). Mitochondrial diseases: The contribution of organelle stress responses to pathology. Nat. Rev. Mol. Cell Biol. 19, 77-92. 
System, E., and Klumperman, J. (2015). The Complex Ultrastructure of the. Cold Spring Harb. Perspect. Biol. 1-22. Tängemo, C., Weber, D., Theiss, S., Mengel, E., and Runz, H. (2011). Niemann-Pick Type C disease: characterizing lipid levels in patients with variant lysosomal cholesterol storage. J. Lipid Res. 52, 813-825.

Thoreen, C.C., Kang, S.A., Chang, J.W., Liu, Q., Zhang, J., Gao, Y., Reichling, L.J., Sim, T., Sabatini, D.M., and Gray, N.S. (2009). An ATP-competitive mammalian target of rapamycin inhibitor reveals rapamycin-resistant functions of mTORC1. J. Biol. Chem. 284, 8023-8032.

Troen, B.R. The role of cathepsin K in normal bone resorption. Drug News Perspect. 17, $19-28$.

Urich, K. (1994). Comparative Animal Biochemistry (Berlin, Heidelberg: Springer Berlin Heidelberg).

Vance, J.E., and Karten, B. (2014). Niemann-Pick C disease and mobilization of lysosomal cholesterol by cyclodextrin. J. Lipid Res. 55, 1609-1621.

Vanier, M.T., and Latour, P. (2015). Laboratory diagnosis of Niemann-Pick disease type C: The filipin staining test (Elsevier Ltd).

Viscomi, C., Bottani, E., Civiletto, G., Cerutti, R., Moggio, M., Fagiolari, G., Schon, E.A., Lamperti, C., and Zeviani, M. (2011). In vivo correction of COX deficiency by activation of the AMPK/PGC-1a axis. Cell Metab. $14,80-90$.

Voelker, D.R. (2005). Bridging gaps in phospholipid transport. Trends Biochem. Sci. 30, 396-404.

Vogel, F., Bornhövd, C., Neupert, W., and Reichert, A.S. (2006). Dynamic subcompartmentalization of the mitochondrial inner membrane. J. Cell Biol. 175, 237-247.

Walkley, S.U., and Vanier, M.T. (2009). Secondary lipid accumulation in lysosomal disease. Biochim. Biophys. Acta - Mol. Cell Res. 1793, 726-736.

Watson, H. (2015). Biological membranes. Essays Biochem. 59, 43-69.

Waypa, G.B., Guzy, R., Mungai, P.T., Mack, M.M., Marks, J.D., Roe, M.W., and Schumacker, P.T. (2006). Increases in Mitochondrial Reactive Oxygen Species Trigger Hypoxia-Induced Calcium Responses in Pulmonary Artery Smooth Muscle Cells. Circ. Res. 99, 970-978.

Wieckowski, M.R., Deus, C.M., Couto, R., Oparka, M., Lebiedzińska-Arciszewska, M., Duszyński, J., and Oliveira, P.J. (2015). Measuring p66Shc Signaling Pathway Activation and Mitochondrial Translocation in Cultured Cells. Curr. Protoc. Toxicol. 66, 25.6.1-25.6.21.

Wong, H.S., Dighe, P.A., Mezera, V., Monternier, P.A., and Brand, M.D. (2017). Production of superoxide and hydrogen peroxide from specific mitochondrial sites under different bioenergetic conditions. J. Biol. Chem. 292, 16804-16809. 
Wyant, G.A., Abu-Remaileh, M., Wolfson, R.L., Chen, W.W., Freinkman, E., Danai, L. V., Vander Heiden, M.G., and Sabatini, D.M. (2017). mTORC1 Activator SLC38A9 Is Required to Efflux Essential Amino Acids from Lysosomes and Use Protein as a Nutrient. Cell 171, 642-654.e12.

Xu, H., and Ren, D. (2015). Lysosomal Physiology. Annu. Rev. Physiol. 77, 57-80.

Yabe, D., Komuro, R., Liang, G., Goldstein, J.L., and Brown, M.S. (2003). Liver-specific mRNA for Insig-2 downregulated by insulin: Implications for fatty acid synthesis. Proc. Natl. Acad. Sci. 100, 3155-3160.

Yambire, K.F., Fernandez-Mosquera, L., Steinfeld, R., Mühle, C., Ikonen, E., Milosevic, I., and Raimundo, N. (2019). Mitochondrial biogenesis is transcriptionally repressed in lysosomal lipid storage diseases. Elife 8, 1-29.

Yancey, P.G., Rodrigueza, W. V, Kilsdonk, E.P., Stoudt, G.W., Johnson, W.J., Phillips, M.C., and Rothblat, G.H. (1996). Cellular cholesterol efflux mediated by cyclodextrins. Demonstration Of kinetic pools and mechanism of efflux. J. Biol. Chem. 271, 16026-16034.

Yang, H., Jiang, X., Li, B., Yang, H.J., Miller, M., Yang, A., Dhar, A., and Pavletich, N.P. (2017). Mechanisms of mTORC1 activation by RHEB and inhibition by PRAS40. Nature 552, 368-373.

Yang, T., Espenshade, P.J., Wright, M.E., Yabe, D., Gong, Y., Aebersold, R., Goldstein, J.L., and Brown, M.S. (2002). Crucial Step in Cholesterol Homeostasis. Cell 110, 489-500.

Yasukawa, T., and Kang, D. (2018). An overview of mammalian mitochondrial DNA replication mechanisms. J. Biochem. 164, 183-193.

Yeagle, P.L. (1985). Cholesterol and the cell membrane. BBA - Rev. Biomembr. 822, 267-287.

Yecies, J.L., Zhang, H.H., Menon, S., Liu, S., Yecies, D., Lipovsky, A.I., Gorgun, C., Kwiatkowski, D.J., Hotamisligil, G.S., Lee, C.-H., et al. (2011). Akt Stimulates Hepatic SREBP1c and Lipogenesis through Parallel mTORC1Dependent and Independent Pathways. Cell Metab. 14, 21-32.

Youle, R.J., and van der Bliek, A.M. (2012). Mitochondrial Fission, Fusion, and Stress. Science (80-. ). 337, 10621065.

Young, M.J., and Copeland, W.C. (2016). Human mitochondrial DNA replication machinery and disease. Curr. Opin. Genet. Dev. 38, 52-62.

Zelcer, N. (2006). Liver $X$ receptors as integrators of metabolic and inflammatory signaling. J. Clin. Invest. 116, $607-614$.

Zidovetzki, R., and Levitan, I. (2007). Use of cyclodextrins to manipulate plasma membrane cholesterol content: Evidence, misconceptions and control strategies. Biochim. Biophys. Acta - Biomembr. 1768, 1311-1324. 
Zimmerberg, J., and Gawrisch, K. (2006). The physical chemistry of biological membranes. Nat. Chem. Biol. 2, 564567.

Zoncu, R., Efeyan, A., and Sabatini, D.M. (2011). mTOR: from growth signal integration to cancer, diabetes and ageing. Nat. Rev. Mol. Cell Biol. 12, 21-35. 


\section{Science \\ DIAAS}

Thank you for your order!

Dear Mr. Leonardo Pergra.

Thank you for placing your ander through Oofyright Clearance Center's RightsLink? servibe.

\section{Order Summary}

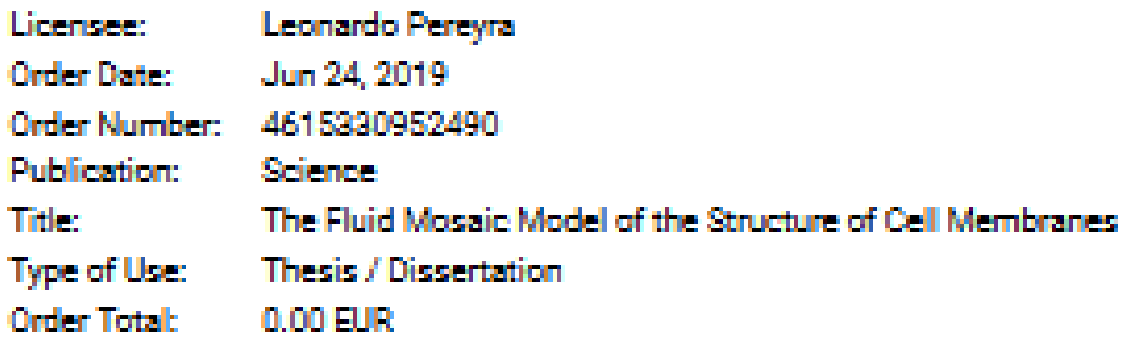

View or print complete details of your crder and the fublisher's tems and anditions.

\section{Sncerely}

Copyight CHearanes Center

TeL +1-695-2393415 I+1-978-649-2777 